\title{
Single-Carrier SM-MIMO: A Promising Design for Broadband Large-Scale Antenna Systems
}

\author{
Ping Yang, Yue Xiao, Yong Liang Guan, Member, IEEE, K. V. S. Hari, Fellow, IEEE, \\ A. Chockalingam, Senior Member, IEEE, Shinya Sugiura, Senior Member, IEEE, Harald Haas, Member, IEEE, \\ Marco Di Renzo, Senior Member, IEEE, Christos Masouros, Senior Member, IEEE, Zilong Liu, Lixia Xiao, \\ Shaoqian Li, Fellow, IEEE, and Lajos Hanzo, Fellow, IEEE
}

\begin{abstract}
The main limitations of employing large-scale antenna (LSA) architectures for broadband frequency-selective channels include, but are not limited to their complexity, power consumption, and the high cost of multiple radio frequency (RF) chains. Promising solutions can be found in the recently proposed family of single-carrier (SC) spatial modulation (SM) transmission techniques. Since the SM scheme's transmit antenna (TA) activation process is carried out in the context of a SCSM architecture, the benefits of a low-complexity and low-cost single-RF transmitter are maintained, while a high MIMO multiplexing gain can be attained. Moreover, owing to its inherent SC structure, the transmit signals of SC-SM have attractive peak power characteristics and a high robustness to $\mathrm{RF}$ hardware impairments, such as the RF carrier frequency offset (CFO) and phase noise. In this paper, we present a comprehensive overview of the latest research achievements of SC-SM, which has recently attracted considerable attention. We outline the associated transceiver design, the benefits and potential tradeoffs, the LSA aided multiuser (MU) transmission developments, the
\end{abstract}

Manuscript received July 3, 2015; revised January 4, 2016; accepted February 14, 2016. This work was supported by the National Science Foundation of China under Grant 61501095, in part by the National HighTech R\&D Program of China ("863" Project under Grant 2014AA01A707), in part by the European Research Council's Advanced Fellow Grant, in part by the Royal Academy of Engineering, U.K. and the Engineering and Physical Sciences Research Council (EPSRC) project EP/M014150/1, and in part by the National Science Foundation of China under Grant 60902026 and Grant 61032002 , and in part by the Japan Society for the Promotion of Science (JSPS) KAKENHI Grant 26709028.

P. Yang, Y. Xiao, L. Xiao, and S. Li are with the National Key Laboratory of Science and Technology on Communications, University of Electronic Science and Technology of China, Sichuan 611731, China (e-mail: yplxw@163.com; xiaoyue@uestc.edu.cn; xiaolixia_cool@163.com; 1sq@uestc.edu.cn).

K. V. S. Hari and A. Chockalingam are with the Department of ECE, Indian Institute of Science, Bangalore 560012, India (e-mail: hêari@ece.iisc.ernet.in; achockal@ece.iisc.ernet.in).

H. Haas is with the Institute for Digital Communications, Joint Research Institute for Signal and Image Processing, School of Engineering, University of Edinburgh, Edinburgh, U.K. (e-mail: h.haas@ed.ac.uk).

M. D. Renzo is with the Laboratoire des Signaux et Systémes, CNRS, CentraleSupélec, Univ Paris Sud, Université Paris-Saclay, Gif-sur-Yvette 91192, France (e-mail: marco.direnzo@12s.centralesupelec.fr).

Y. Guan and Z. Liu are with the School of Electrical and Electronic Engineering, Nanyang Technological University, Singapore 639798 (e-mail: EYLGuan@ntu.edu.sg; zilongliu@ntu.edu.sg).

S. Sugiura is with the Department of Computer and Information Sciences, Tokyo University of Agriculture and Technology, Koganei, Tokyo 184-8588, Japan (e-mail: sugiura@ieee.org).

C. Masouros is with the Communications and Information Systems Research Group, Department of Electrical and Electronic Engineering, University College London, London, U.K. (e-mail: c.masouros@ucl.ac.uk).

L. Hanzo is with the School of Electronics and Computer Science, University of Southampton, Southampton SO17 1BJ, U.K. (e-mail: 1h@ecs.soton.ac.uk).

Digital Object Identifier 10.1109/COMST.2016.2533580 relevant open research issues as well as the potential solutions of this appealing transmission technique.

Index Terms-Energy efficiency, frequency selective channels, large-scale MIMO systems, spatial modulation, single-carrier transmission, single-RF chain, multiuser communication.

\section{INTRODUCTION}

$\mathbf{M}$ ULTIPLE-INPUT multiple-output (MIMO) techniques have been widely studied during the last two decades and have been incorporated into most of the recent communication standards [1], [2] due to their benefits of providing diversity, and/or multiplexing gains [3]-[5]. However, in order to satisfy the ever-growing demands for higher capacity, larger coverage area and better reliability, further improved MIMO transceivers should be designed [6].

Pursuing this research objective, the concept of large-scale MIMO (LS-MIMO) techniques has been proposed [6]-[12], which employs tens to hundreds of transmit/receive antennas for dramatically improving the attainable link reliability, spectral efficiency and cellular coverage. Usually, the multipleantenna front-end architecture requires a separate radio frequency (RF) chain. This means that the cost of MIMO transmitters to scale linearly with the number of antennas [13]. Moreover, the presence of hundreds of antennas will increase the complexity of both the transmit signal generation and detection. Furthermore, the RF power amplifiers and the associated high-dimensional signal processing may erode the energy efficiency of systems, which are responsible for about 55$85 \%$ of the total power consumption [14], [15]. These factors exacerbate the practical realization of LS-MIMO systems.

The emerging SM design paradigm constitutes an attractive low-complexity yet energy-efficiency option for the family of LS-MIMO systems, due to its low-cost single-RF-based transmitter, as well as owing to its low-complexity single-stream based detector and its ability to maintain the potential benefits of multiple antennas [16]-[19]. The roots of the SM design philosophy can be traced back to the combination-based frequency hopping code division multiple access technique (FH-CDMA) proposed in 1980 [20]-[22], where the combination pattern of the frequency bins was used for conveying information, hence resulting in an improved throughput. The first conference paper on single-RF based SM was published in 2006 [23], but its extensive research was mainly fueled by the pioneering works of Haas et al. [16]-[18], Mesleh et al. [16], [24], [25], followed 
by Yang et al. [26], Sugiura et al. [27], Renzo et al. [28]-[32], Panayırcı and Poor et al. [33], Başar [34], Chang et al. [35], [36] as well as Jeganathan et al. [37]. Throughout its decadelong history, the SM concept has been termed in different ways and it was extended to different scenarios. The wide-ranging studies disseminated in [23]-[42] have characterized some of the fundamental properties of SM. For example, the attainable bit error rate (BER) performance in different fading channels [23], [29]-[32], [37]-[39], the issues of achieving transmit diversity [27], [28], [31], [40], the effects of channel estimation errors [32], [41], [42], as well as the effects of power imbalance [43] have been characterized. Moreover, some simplified and generalized SM schemes have also been proposed by exploiting the different degrees of freedom offered by the temporal domain, frequency domain and spatial domain fading [17][19], [27], such as the space shift keying (SSK), the generalized SSK (GSSK), the generalized SM (GSM), the space-time shift keying (STSK), the space-frequency shift keying (SFSK) and the space-time-frequency shift keying (STFSK) arrangements. It was found that SM and its variants are capable of striking a flexible tradeoff amongst the computational complexity imposed, the number of RF chains required, the attainable BER and the achievable transmission rate.

To be specific, SM relies on the unique encoding philosophy of activating one out of numerous transmit antennas (TAs) during each channel-use [23]-[31]. The activated TA then transmits classic complex-valued symbols of amplitude and phase modulation (APM). Since in SM only a single TA is activated at any time instant, its transmitted signal is sparse in the spatial domain. The advantages of SM-MIMO include its low-dimensional signal representation as well as reduced RF hardware complexity, cost, and size [18], [19]. Moreover, it is capable of relaxing the requirement of inter-antenna synchronization and of mitigating the inter-antenna interference of conventional MIMO schemes. Apart from the earlier variants and extensions of SM disseminated in [17]-[19], recently a variety of SM-related classes have been proposed, including those designed for spectrum sharing environments [44], for multiple access transmissions [45], for energy-efficient applications [46], for cooperative SM scenarios [47]-[53] and for closedloop designs [54]. The above-mentioned potential benefits of SM over the conventional MIMO techniques have been validated not only via numerical simulations [16], [23], [29]-[32], [37]-[39] but also by laboratory experiments [55]-[57].

Despite of its rich literature [23]-[37], the family of SMrelated schemes has been predominantly investigated in the context of flat fading channels. In the case of wide-band frequency-selective channels, orthogonal frequency-division multiplexing (OFDM) [58] is generally assumed in SM-related schemes. In this case, multiple TAs have to be simultaneously activated over the OFDM frame, which will jeopardize most of the single-RF benefits of SM-MIMO. Moreover, the OFDM signal has a high peak-to-average power ratio (PAPR), which may result in a low power amplifier efficiency [59]. Furthermore, the computational burden of performing the discrete Fourier transform (DFT) of the OFDM scheme increases with the number the TAs. Due to these reasons, the OFDMbased SM (OFDM-SM) schemes [60] and the related variants may not be suitable for the family LS-MIMO.

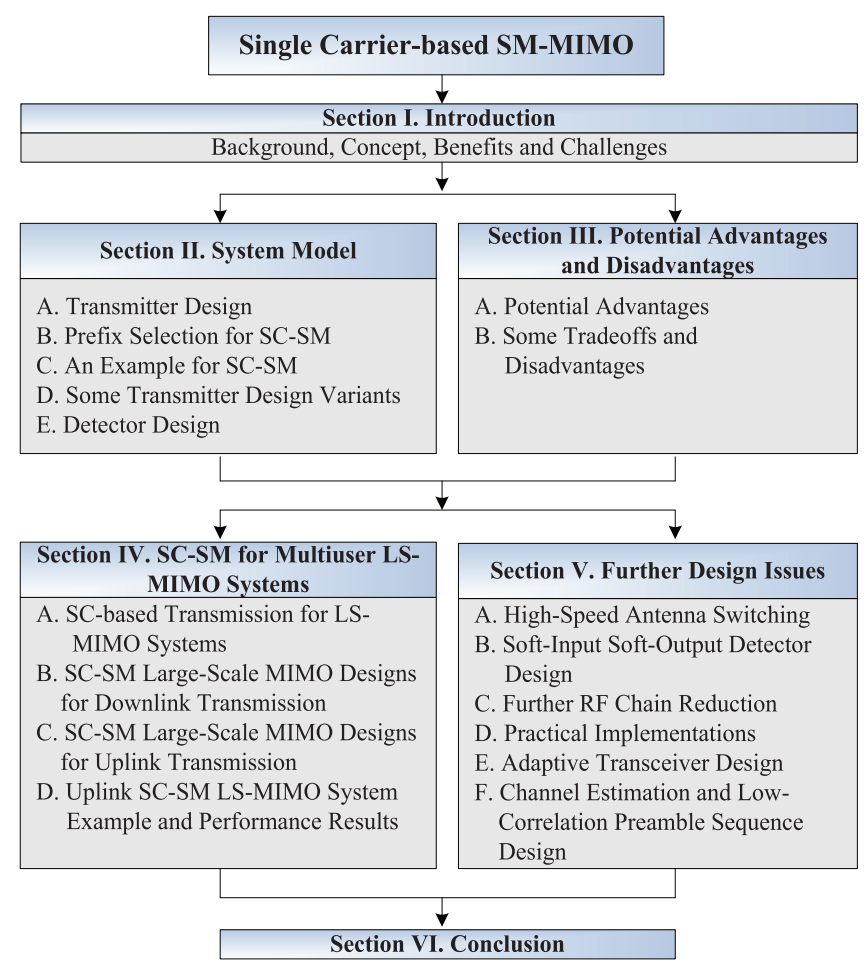

Fig. 1. Structure of this survey paper.

In recent years, the family of single-carrier (SC) or SC- 128 like transmissions has become an interesting and promising 129 alternative for LS-MIMO, which can reduce the complexity 130 imposed by the DFT and has a lower PAPR than OFDM [61]- 131 [64]. Moreover, as shown in [10], [65], the frequency-selective 132 channel may be converted into a frequency-flat channel in 133 LS-MIMO by carefully designed receive filters and therefore 134 SC-based scheme can be directly used.

Motivated by the above-mentioned appealing characteristics of both SM and SC techniques shown in LS-MIMO transmissions, a flurry of research activities on SC-based SM (SC-SM) designs have been sparked off. In this paper we provide an overview of the broadband SC-SM architecture, which has the potential of exploiting the joint advantages of both SM and SC techniques. We summarize recent results concerning this novel transmission technique and outline the associated transceiver design, as well as a range of potential solutions in the scenario of LS-MIMO, highlighting its impediments as well as its future research directions to consider.

\section{A. Outline}

The remainder of this paper is organized as shown in Fig. 1. 148 Section II introduces the system model of the SC-SM technique 149 and its relevant variants, emphasizing the transmitter design, 150 the prefix selection and various detector designs. In particu- 151 lar, both the time-domain and the frequency-domain as well 152 as the associated turbo equalizers are investigated. The poten- 153 tial advantages and disadvantages of the SC-SM scheme are 154 summarized in Section III. In Section IV the applications of 155 the SC-SM transceiver to LS-MIMO systems are presented, 156 where different SC-SM designs conceived for the multiuser LS- 157 MIMO uplink and downlink are reviewed. Section V highlights 158 
a range of design issues that require more intensive study. Finally, Section VI concludes the paper.

\section{B. Notations}

The following notations are employed throughout this contribution. $(\cdot)^{T}$ and $(\cdot)^{H}$ denote the matrix transpose and Hermitian transpose, respectively. The probability of an event is represented by $p(\cdot)$. Furthermore, $\|\cdot\|$ and $|\cdot|$ denote the Euclidean norm and magnitude operators, while $U$ is the number of users, $N_{\text {tot }}$ is the number of antennas at the BS for its MU transmission and $N_{\mathrm{u}}$ is the number of antennas at each user for their multiuser transmission. Furthermore, $N_{t}$ and $N_{r}$ are the number of the antennas at transmitter and receiver for their point-topoint transmission, respectively, while $M_{\mathrm{SM}}, M_{\mathrm{VBLAST}}$ and $M_{\mathrm{STBC}}$ are the sizes of the PSK/QAM constellation used in SM, space time block code (STBC) and vertical Bell labs layered space-time (VBLAST), respectively; $K$ is the length of the transmission frame; $\mathbf{b}$ is the transmitted bit vector; $\mathbf{e}_{i}$ is the natural basis vector with only a single nonzero element at $i$ th position; $L$ is the maximum channel impulse response duration; $v$ is the length of the prefix of the transmit SC frame and $\mathbf{x}$ is the SM transmit symbol vector.

\section{System Model OF SC-SM}

\section{A. Transmitter Design}

In this section, we consider a broadband SC-SM based MIMO system employing $N_{t}$ TAs as well as $N_{r}$ receive antennas (RAs) and communicating over a frequency-selective fading channel, as depicted in Fig. 2. In a conventional narrowband SM scheme, the information bits are conveyed by the indices of the TAs as well as the classic APM constellation (i.e. $M_{\mathrm{SM}}$-PSK/QAM). Specifically, one of the $N_{t}$ TA elements is activated at each time slot by $\log _{2}\left(N_{t}\right)$ information bits, while one of $M_{\mathrm{SM}}$-APM symbols is selected by another $\log _{2}\left(M_{\mathrm{SM}}\right)$ information bits, which will be transmitted by the activated TA. In other words, the numbers of bits conveyed by the TA indices and APM symbols are $\log _{2}\left(N_{t}\right)$ and $\log _{2}\left(M_{\mathrm{SM}}\right)$, respectively. In total, $m_{\mathrm{SM}}=\log _{2}\left(N_{t} M_{\mathrm{SM}}\right)$ bits are sent over one SM symbol.

In broadband SC-SM, each SC transmit block is composed by the prefix part and the data part. The data part contains $K$ SM symbols, each of which consisting of $m_{\mathrm{SM}}$ bits. Hence, a total of $m_{r}=K m_{\mathrm{SM}}$ bits are conveyed over an SC-SM symbol. We assume that there are $L$ resolvable multipath links between each TA and RA pair, an $M_{\mathrm{SM}}$-PSK/QAM scheme is adopted, and $N_{t}$ is a power of two ${ }^{1}$. Generally, in SC-SM based MIMO transmission [66], each transmit block can be generated by the following three steps:

The pseudo-code of Algorithm 1 is given in Table I. An example of conventional SM mapping rule for $N_{t}=2$ employing BPSK modulation is portrayed in Fig. 2. In this system, the number of bits conveyed by each SM symbol is $m_{\mathrm{SM}}=\log _{2}\left(N_{t} M_{\mathrm{SM}}\right)=2$. All possible two-bit combinations

\footnotetext{
${ }^{1}$ This restriction can be solved by the non-integer-based encoding method of [67] and the bit padding method of [68].
}

Algorithm 1. Generation of a Transmit Frame According to SM Mapping Rule

1) First, the information bit stream is divided into vectors containing $m_{r}=K m_{\mathrm{SM}}$ bits each, which will be transmitted over one SC-SM frame. Furthermore, each vector is split into $K$ sub-vectors $\mathbf{b}(k), k=\{1, \cdots, K\}$ having length of $m_{\mathrm{SM}}=\log _{2}\left(N_{t} M_{\mathrm{SM}}\right)$.

2) Next, each sub-vector $\mathbf{b}(k), k \in\{1, \cdots, K\}$ is mapped to an SM symbol. Specifically, based on the SM principle, each $\mathbf{b}(k)$ is divided into two sequences of $\log _{2}\left(N_{t}\right)$ and $\log _{2}\left(M_{\mathrm{SM}}\right)$ bits, denoted by $\mathbf{b}_{1}(k)$ and $\mathbf{b}_{2}(k)$, respectively. The bits in $\mathbf{b}_{1}(k)$ are used for selecting a unique TA index $q(k)$ for activation, which is mapped to an $N_{t}$-dimensional natural basis vector $\mathbf{e}_{q(k)}$ (i.e., $\mathbf{e}_{1}=[1,0, \cdots, 0]^{T}$ ), while the bits in $\mathbf{b}_{2}(k)$ are mapped to an $M_{\mathrm{SM}}$-APM (i.e. PSK/QAM) constellation point $s_{l}^{q(k)}$. For simplicity, we refer to these two sets of bits as TA-bits and APM-bits. The resultant SM symbol based on the sub-vector $\mathbf{b}(k), k \in$ $\{1, \cdots, K\}$ can be formulated by

$$
\mathbf{x}(k)=[\underbrace{0, \cdots, 0}_{q(k)-1}, s_{l}^{q(k)}, \underbrace{0, \cdots, 0}_{N_{t}-q(k)}]^{T} \in \mathbb{C}^{N_{t} \times 1},
$$

where $q(k), k \in\{1, \cdots, K\}$ is the index of the activated TA during the $k$ th interval. The corresponding transmit block of the $K$ SM-mapped symbols is formulated as $\mathbf{X}$ $=[\mathbf{x}(1), \cdots \mathbf{x}(k), \cdots, \mathbf{x}(K)]^{T} \in \mathbb{C}^{N_{t} \times K}$.

3) Finally, for each TA, a specific prefix vector $\mathbf{w}=[w(1), \cdots \cdots, w(v)]^{T} \in \mathbb{C}^{1 \times v}$, i.e. the zero-padding (ZP) or cyclic prefix (CP) vector, is inserted for preventing the inter-block interference (IBI), which has to have a length larger than or equal to the maximum channel impulse response (CIR) duration $L$. Then, the SC-SM signal block and its prefix is transmitted over $(K+v)$ consecutive symbols durations.

$\{00,01,10,11\}$ are mapped to the TA indices $\{1,2\}$ and to the 210 BPSK constellation $\{-1,+1\}$, e.g. TA 1 is activated to trans- 211 mit the BPSK constellation point -1 when the input bits are 212 00. According to Algorithm 1, at the receiver side, $\mathbf{y}(k)=213$ $\left[y_{k, 1}, \cdots, y_{k, N_{r}}\right]^{T}$, where $y_{k, j}$ is the received signal on $j$ th RA 214 at time slot $k$, is given by

$$
\mathbf{y}(k)=\sum_{l=0}^{L-1} \mathbf{H}_{l} \mathbf{x}(k-l)+\boldsymbol{\eta}(k),
$$

where $\mathbf{H}_{l}$ is an $N_{r}$-by- $N_{t}$ matrix with the entry $h_{i j}^{l}$ being the 216 $l$ th CIR of the link spanning from the $j$ th TA to the $i$ th RA. 217 Moreover, $\boldsymbol{\eta}(k) \in \mathbb{C}^{N_{r} \times 1}$ is the noise vector, whose elements 218 are complex Gaussian random variables obeying $\mathcal{C} \mathcal{N}\left(0, N_{0}\right) .219$ To elaborate a little further, we will provide an example of SC- 220 $\mathrm{SM}$ in Section II-C.

\section{B. Prefix Selection for SC-SM}

As shown in Fig. 2, in order to suppress IBI, each SC block 223 is extended by a guard interval, termed as the prefix vector. In 224 


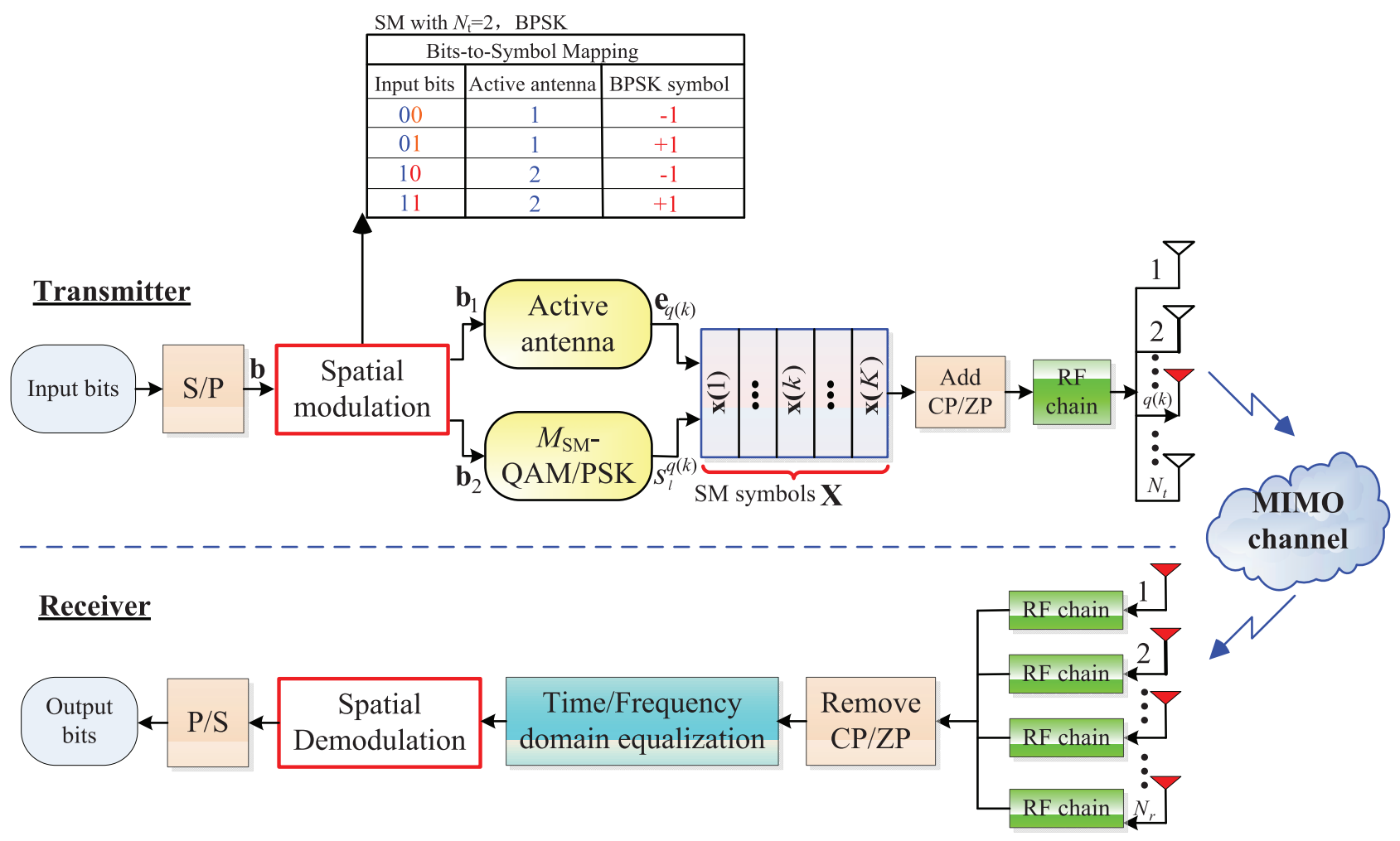

Fig. 2. General transceiver structure of SC-SM systems.

TABLE I

Generation of AN SC-SM TRANSMit Frame ACCORding to SM MAPPING RULE

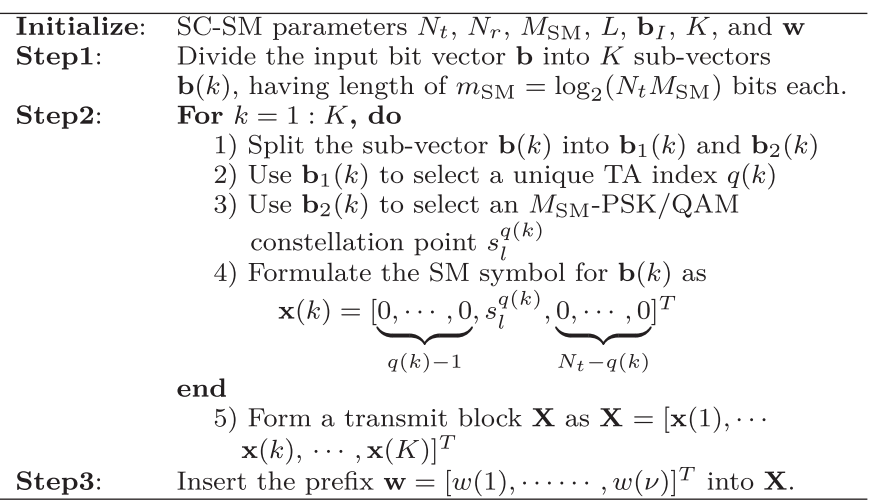

conventional SC-based MIMO systems, the commonly adopted guard interval schemes may be roughly divided into four fundamental types [61], [62], as illustrated in Fig. 3, where both the attainable transmission rate and the power efficiency of an SC-based system are highly dependent on the specific type of the guard interval used.

- CP scheme: The last samples of each transmit block of $K$ samples are copied to the beginning of this transmit block. Apart from the elimination of the IBI from the previous block, it also transforms the linear convolution of a frequency-selective multipath channel into a circular one and thus permits low-complexity channel diagonalization in the frequency domain at the receiver. However,

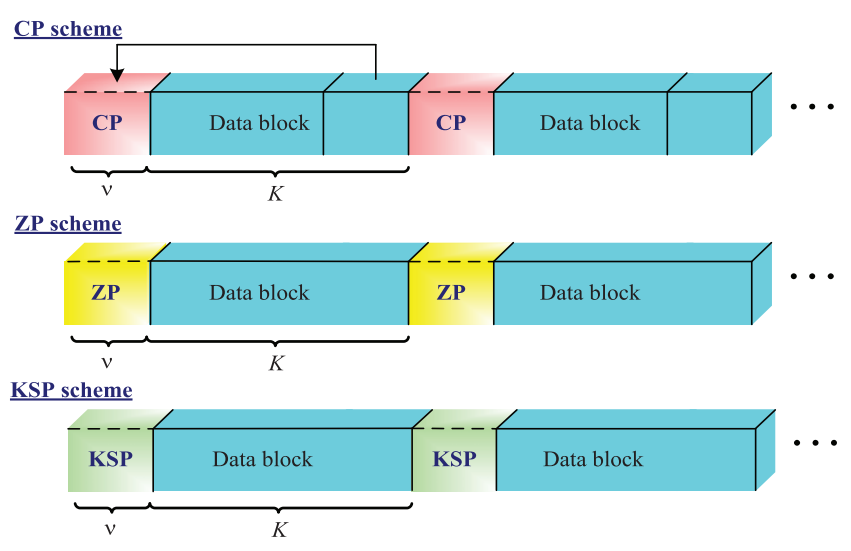

Non-prefix scheme

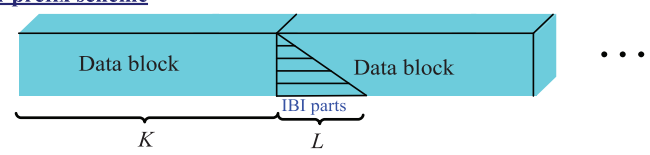

Fig. 3. Guard interval schemes for SC-based MIMO schemes in frequencyselective channels.

the CP scheme suffers from a power efficiency reduction 238 according to the factor of $K /(K+v)$.

- ZP scheme: A guard interval of samples is padded after 240 each transmit block, during which no signal is trans- 241 mitted. It can avoid the power efficiency erosion of the 242 CP scheme. Moreover, it supports a linear receiver in 243 achieving full multipath diversity in some transmission 244 scenarios [61]. The price paid is a somewhat increased 245 receiver complexity. 
- Known symbol padding (KSP) scheme: A guard interval consisting of a sequence of known samples is added after each transmit block. These known samples may represent a carefully designed sequence, such as a pseudo noise sequence [62]. In case of both $\mathrm{CP}$ and $\mathrm{ZP}$ schemes, data-aided channel estimation is performed by replacing some data symbols with pilot symbols, while the known symbols of KSP inserted into the guard interval can be directly used as pilot symbols. Note however that the KSP scheme also suffers from a power efficiency loss.

- Non-prefix scheme: The employment of the prefix vector imposes both a power and a spectral overheads, especially in case of a short channel coherence time and long CIR. To mitigate power and spectral overheads, the IBI can be reduced by using a powerful receiver even in the absence of a prefix vector, where iterative detection may be used. Moreover, to avoid the overhead imposed by the pilot signals, implicit training [69] can be adopted.

As a new SC-based MIMO technique, a suitable prefix vector should be carefully selected such that a specific SC-SM scheme can satisfy a diverse range of practical requirements. In [70], the authors exploited the classic CP scheme in the context of an SC-SM scheme and provided a diversity analysis framework. It was shown in [70] that the diversity order achieved by the CP-aided SC-SM under maximum-likelihood (ML) detection is only determined by the number of RAs $N_{r}$. Moreover, it was shown in [70] that their proposed SC-SM scheme is capable of outperforming its OFDM-based counterpart. However, the CPaided SC-SM scheme suffers from a high detection complexity as well as a multipath diversity gain erosion. To be specific, in such a case, the ML complexity of this scheme is in the order of $O\left(N_{t} M_{\mathrm{SM}}\right)^{K}$ and no multipath diversity gain can be achieved.

More recently, Rajashekar et al. [71] further generalized the solutions of [70], where a ZP-based SC-SM was proposed for reducing the order of ML detection complexity from $\left(N_{t} M_{\mathrm{SM}}\right)^{K}$ to $\left(N_{t} M_{\mathrm{SM}}\right)^{L}$, with $L$ being the length of the CIR. Moreover, it was shown in [71] that the ZP-aided SC-SM scheme offers a full receive and multipath diversity order of $L N_{r}$ and hence achieves significant performance improvement compared to both the CP-aided OFDM-SM and the CP-aided SC-SM schemes.

\section{An Example for SC-SM}

As an example, let us consider a $(2 \times 2)$-element MIMO transmission $\left(N_{t}=N_{r}=2\right)$ associated with the throughput of $m_{\mathrm{MIMO}}=4 \mathrm{bits} / \mathrm{channel-use}$ (bpcu). We are interested in the comparison of SM with respect to other MIMO arrangements, such as the classic STBC and VBLAST schemes. In Fig. 4, five different MIMO schemes, namely SC-SM, OFDM-SM, SC-VBLAST, OFDM-VBLAST and OFDM-STBC, are considered. Note that in order to achieve the identical throughput, we consider 8-PSK, QPSK, and 16-QAM constellations for the SM-based, VBLAST, and the classic Alamouti STBC schemes [72], respectively. In all OFDM-based schemes, only the classic $\mathrm{CP}$ vector is inserted. For the sake of simplicity, we assume that the CIR length is $L=3$ and the frame length is $K=4$. Hence, the $K$ MIMO symbols of a single frame convey a total of $\left(K \times m_{\mathrm{MIMO}}\right)=(4 \times 4)=16$ bits. As shown in Fig. 4 , the 303 information bit stream is divided into sub-vectors containing 304 16 bits each. Assuming that the current transmit sub-vector is 305 $\mathbf{b}=[1011001000101110]$, we present the details of the transmit 306 vector generation of these five MIMO schemes as follows: 307

1) SC-SM: In SM, the throughput is $m_{\mathrm{MIMO}}=308$ $\log _{2}\left(N_{t} M_{\mathrm{SM}}\right)$, which is achieved by using 8-PSK 309 $\left(M_{\mathrm{SM}}=8\right)$ in our example in conjunction with 310 $N_{t}=2$. According to the SC-SM model detailed in 311 Fig. 2, the sub-vector $\mathbf{b}=[1011001000101110]$ is 312 further split into $K=4$ sequences, i.e. $\mathbf{b}(1)=[1001], 313$ $\mathbf{b}(2)=[0000], \mathbf{b}(3)=[1101]$, and $\mathbf{b}(4)=[1110]$. Then, 314 each sequence $\mathbf{b}(k), k \in\{1, \cdots, 4\}$ is divided into 315 $\log _{2}\left(N_{t}\right)=\log _{2}(2)=1$ and $\log _{2}\left(M_{\mathrm{SM}}\right)=\log _{2}(8)=3 \quad 316$ bits. Specifically, the sequence $\mathbf{b}(1)=[1001]$ is divided 317 into $\mathbf{b}_{1}(1)=[1]$ and $\mathbf{b}_{2}(1)=[001], \mathbf{b}(2)=[0000]$ is 318 divided into $\mathbf{b}_{1}(2)=[0]$ and $\mathbf{b}_{2}(2)=[000], \mathbf{b}(3)=[1101] 319$ is divided into $\mathbf{b}_{1}(3)=[1]$ and $\mathbf{b}_{2}(3)=[101], 320$ and $\mathbf{b}(4)=[1110]$ is divided into $\mathbf{b}_{1}(4)=[1]$ and 321 $\mathbf{b}_{2}(4)=[110]$. Thus, the bits conveyed by the TA 322 index are $\mathbf{b}_{1}=\left[\mathbf{b}_{1}(1), \mathbf{b}_{1}(2), \mathbf{b}_{1}(3), \mathbf{b}_{1}(4)\right]=[1011] 323$ and the bits conveyed by 8-PSK signals are 324 $\mathbf{b}_{2}=\left[\mathbf{b}_{2}(1), \mathbf{b}_{2}(2), \mathbf{b}_{2}(3), \mathbf{b}_{2}(4)\right]=[001000101110] . \quad 325$ After this split, $\mathbf{b}(k), k \in\{1, \cdots, 4\}$ are mapped to 326 the conventional SM symbols. For example, for the 327 sequence $\mathbf{b}(1)=\left[\mathbf{b}_{1}(1), \mathbf{b}_{1}(2)\right], \mathbf{b}_{1}(1)=[1]$ is used to 328 activate TA 2 and $\mathbf{b}_{2}(1)=[001]$ is mapped to the 8-PSK 329 constellation point $e^{\frac{\pi}{4} i}$, which is transmitted over TA 2. 330 Hence, the corresponding SM symbol for transmission 331 is $\mathbf{x}(1)=\left[0, e^{\frac{\pi}{4} i}\right]^{T}$. Similarly, we can also generate the 332 transmit SM symbols $\mathbf{x}(2), \mathbf{x}(3)$ and $\mathbf{x}(4)$ corresponding 333 to $\mathbf{b}(2), \mathbf{b}(3)$ and $\mathbf{b}(4)$, respectively. The resultant 334 SC-SM transmit frame is formulated by $\mathbf{X}=[\mathbf{x}(1), \mathbf{x}(2), \quad 335$ $\mathbf{x}(3), \mathbf{x}(4)]^{T}$. Finally, the ZP or CP vector is inserted by 336 using the method shown in Fig. 3.

2) OFDM-SM: The initial bit-to-symbol mapping process of 338 OFDM-SM is the same as that of the SC-SM scheme, 339 except that the transmit signal $\mathbf{X}$ is considered to be a 340 frequency-domain signal and an inverse fast Fourier trans- 341 form (IFFT) unit is used to produce the corresponding 342 complex-valued time-domain signal, e.g., the frequency- 343 domain vector [ $\left[\begin{array}{llll}0 & 1 & 0 & 0\end{array}\right]$ transmitted over TA 1 is converted 344 to its time-domain signal as $[0.5,0.5 i,-0.5,0.5 i]$. As 345 shown in Fig. 4, the OFDM-SM signal generated is no 346 longer sparsely distributed in the spatial domain and two 347 TAs (two RF chains) have to be simultaneously activated 348 over the OFDM frame. Fig. 4 shows an example of the 349 OFDM-SM signal transmission for the first symbol of 350 $[0.5,0.35+0.35 i]$.

3) SC-VBLAST: In VBLAST, each TA simply transmits 352 an independent symbol stream and the throughput is 353 $m_{\mathrm{MIMO}}=N_{t} \log _{2}\left(M_{\mathrm{VBLAST}}\right)$, where $M_{\mathrm{VBLAST}}$ repre- 354 sents the order of APM constellation by the VBLAST 355 scheme. In our example associated with $m_{\mathrm{MIMO}}=4 \mathrm{bpcu} 356$ and $N_{t}=2$, we have $M_{\text {VBLAST }}=4$ and hence a QPSK 357 modulation is used. As shown in Fig. 4, the bit partition- 358 ing of the input bit stream $\mathbf{b}=[1011001000101110]$ is 359 different from that in SM. Specifically, b is first equally 360 

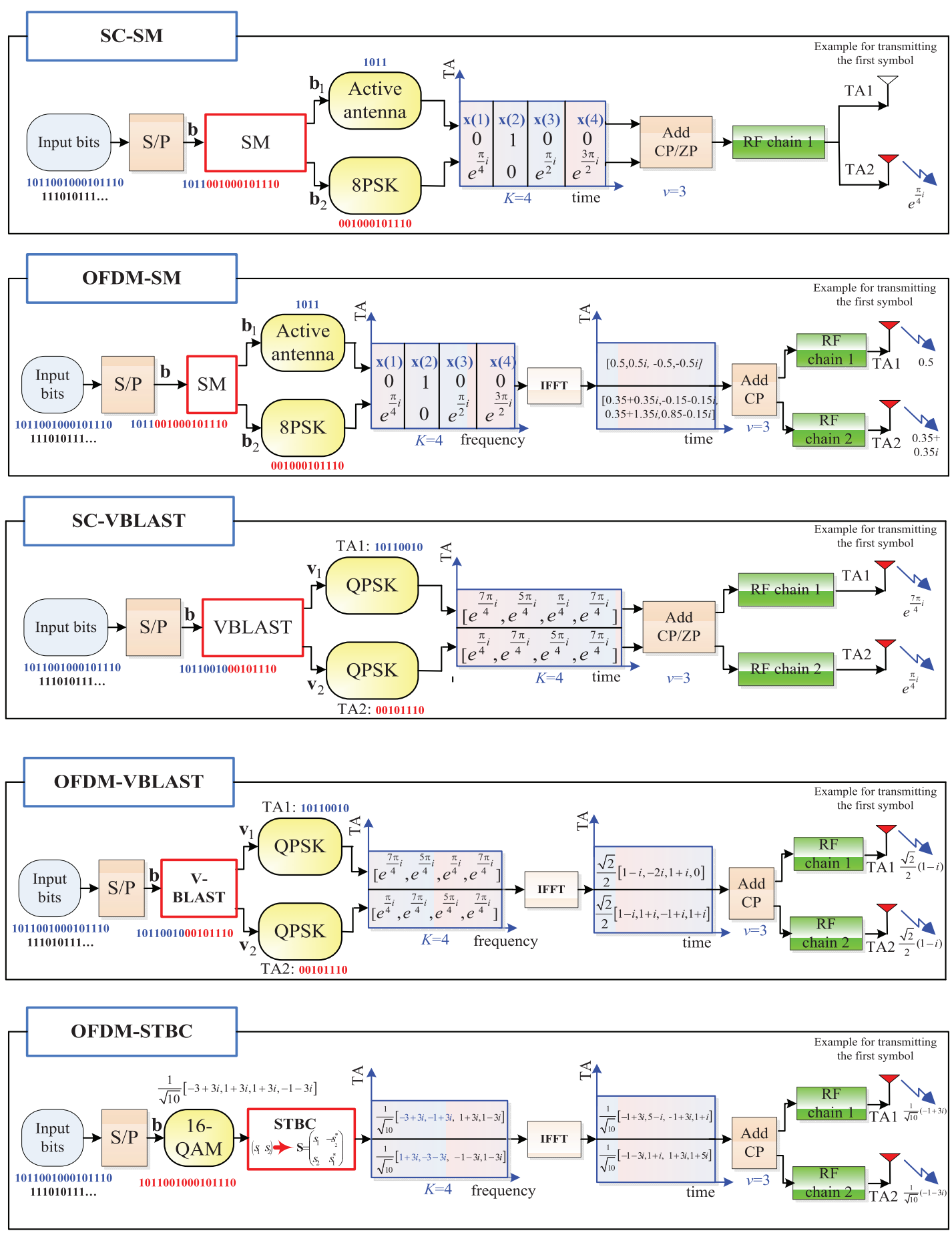

Fig. 4. Examples of different MIMO transmitters for multipath channels at a throughput of 4 bits/channel-use, where the SC-SM, the OFDM-SM, the SCVBLAST, the OFDM-VBLAST and the OFDM-STBC schemes are considered.

partitioned into two sub-vectors $\mathbf{v}_{1}=[10110010]$ and $\mathbf{v}_{2}=[00101110]$ and then further divided into $K=4$ sequences. For example, $\mathbf{v}_{1}=[10110010]$ is split into [10], [11], [00] and [10], which will be mapped to the QPSK symbols of $e^{\frac{7 \pi}{4} i}, e^{\frac{5 \pi}{4} i}, e^{\frac{\pi}{4} i}, e^{\frac{7 \pi}{4} i}$, respectively. The transmited SC-VBLAST vector of TA 1 is given by $\left[e^{\frac{7 \pi}{4} i}\right.$, $\left.e^{\frac{5 \pi}{4} i}, e^{\frac{\pi}{4} i}, e^{\frac{7 \pi}{4} i}\right]$. The same process can be carried out for TA 2. Finally, the ZP or CP vectors for the generated transmit vector of each TA are inserted.
4) OFDM-VBLAST: Based on the signal generation steps of 370 SC-VBLAST, we need to add an IFFT unit to produce 371 the OFDM-VBLAST transmit signal, as shown in Fig. 4. 372 After this, such a signal will be sent out over multiple 373 sub-carriers, which are orthogonal to each other. 374

5) OFDM-STBC: The throughput of STBC depends on 375 its code rate (or the multiplexing gain) $c_{\mathrm{STBC}}$ and is 376 given as $m_{\mathrm{MIMO}}=c_{\mathrm{STBC}} \log _{2}\left(M_{\mathrm{STBC}}\right)$, where $M_{\mathrm{STBC}} 377$ is the order of the APM used by the STBC scheme. 378 In our example associated with $N_{t}=2$, the classic 379 


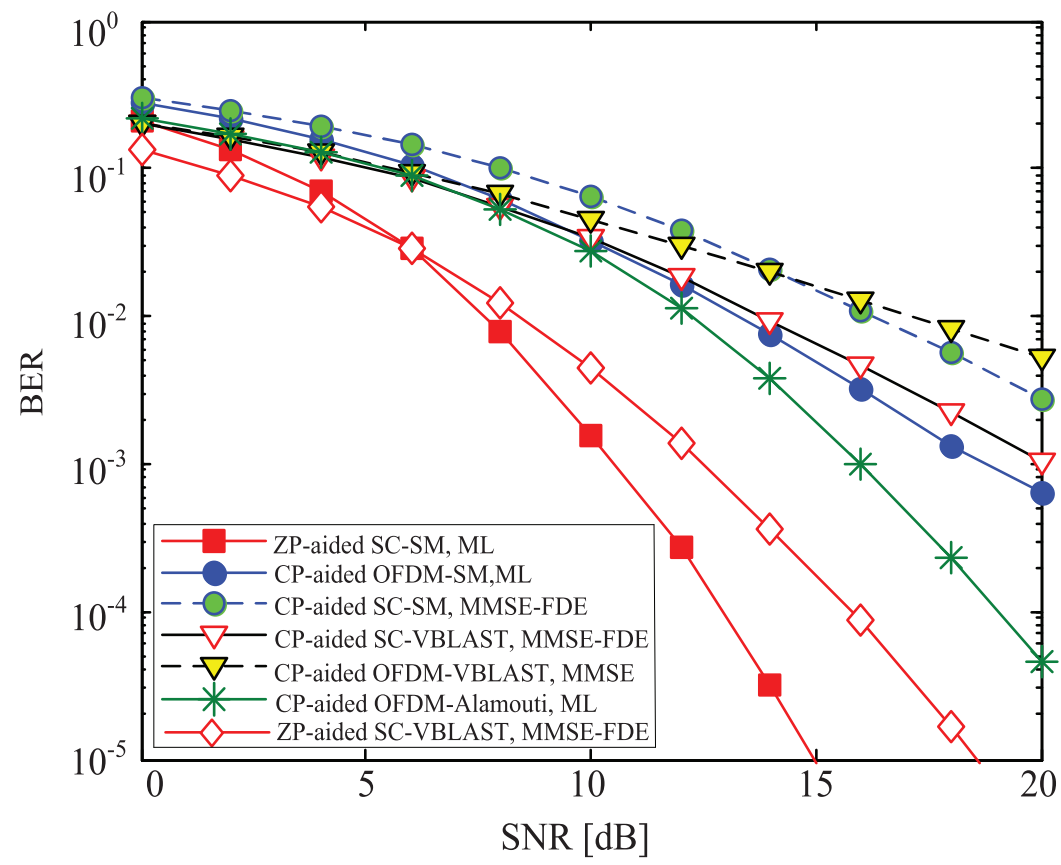

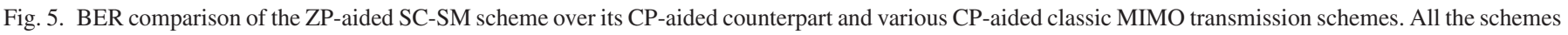

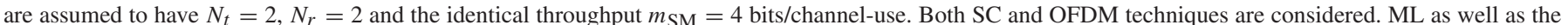
MMSE-type detectors are employed.

Alamouti STBC with $c_{\mathrm{STBC}}=1$ is used and 16QAM $\left(M_{\mathrm{STBC}}=16\right)$ is employed to achieve the target throughput of $m_{\mathrm{MIMO}}=4 \mathrm{bpcu}$. In contrast to the VBLAST and SM schemes, the input bit stream $\mathbf{b}=$ [1011001000101110] is directly mapped to four 16-QAM symbols as in $\frac{1}{\sqrt{10}}[-3+3 i, 1+3 i, 1+3 i,-1-3 i]$. Then, the Alamouti encoding matrix is applied to every pair of symbols and an IFFT unit converts the frequencydomain signal into its time-domain counterpart.

Based on the transmit models of Fig. 4, we compared the BER performance of SC-SM schemes against that of the conventional MIMO schemes in Fig. 5 over Rayleigh fading channels having a uniform power delay profile [71]. In this context, several commonly used detectors are considered, i.e. ML detector, single-tap based minimum mean-squared error (MMSE) detector or MMSE based frequency-domain equalization (FDE) [66], [70], [71].

As shown in Fig. 5, the ZP-aided SC-SM scheme using the ML detector is capable of achieving a considerable diversity gain and hence it attains the best BER performance amongst all benchmark schemes. As pointed out in in [62], the ZP-type prefix vector results in a better BER compared to its CP counterpart, since in the latter case any data detection errors may affect both the information symbols and the CP symbols. In this comparison, we only consider a small frame length $K$ for illustration purpose. In [71] and [88], more comparisons of the SC-SM schemes over other MIMO schemes are provided for larger $K$, in which the afore-mentioned BER benefits were also observed.

Moreover, in Table II, the complexity orders of different ZP-aided and CP-aided MIMO schemes are compared, where only the multiplications of complex numbers are counted.
The complexity orders of different detectors for OFDM-SM, 412 OFDM-STBC and OFDM-VBLAST schemes can be found in 413 [16], while that of the other MIMO schemes can be found in 414 [71] and [88]. In Table II, we also provide the complexity order 415 of the near ML detector, namely low-complexity single-stream 416 (LSS) detector [88], for the promising ZP-aided SC-SM. The 417 details about the near-ML LSS detector will be further dis- 418 cussed in Section II-D. As shown in Table II, the CP-aided 419 MIMO systems with the MMSE and the MMSE-FDE based 420 detectors exhibit lower complexity orders compared to those 421 of ZP-aided MIMO systems with time-domain detectors (ZP- 422 aided SC-VBLAST with MMSE detector and ZP-aided SC-SM 423 with LSS detector), since these CP-aided systems can use low- 424 complexity one-tap equalizations. However, as shown in our 425 simulation results, CP-aided MIMO systems suffer from a mul- 426 tipath diversity gain loss. As proved in [71], the ZP-aided 427 SC-SM scheme with ML detector is capable of offering full 428 multipath diversity and hence exhibits better BERs shown in 429 Fig. 5. This benefit can also be achieved by using the LSS algo- 430 rithm proposed in [88], as will be shown in Figs. 7 and 8 in 431 Section II-D.

By taking into account both the BER and the complexity, 433 we conclude that ZP-aided SC-SM is a promising candidate for 434 dispersive MIMO channels. Note that the detection complexity 435 of ZP-aided SC-SM may be further reduced by exploiting the 436 spatial-domain sparsity of SM symbols, as discussed in [88] and 437 Section III. Moreover in Table II, we also provide the number 438 of RF chains required for different MIMO schemes in disper- 439 sive channels. It is shown in Table II that the SC-SM schemes 440 require only a single RF chain at the transmitter. 441

Note that although the above-mentioned research demon- 442 strated that the ZP based scheme constitutes a promising prefix 443 
TABLE. II

COMPleXity ORders AND THE Number of RF CHAINS REQUiRED FOR DifFERENT ZP-AIDED AND CP-AIDED MIMO SCHEMES

\begin{tabular}{|l|l|l|l|}
\hline Scheme & Detector & Complexity Order (Flops) & RF Chains \\
\hline \hline \multirow{2}{*}{ ZP-aided SC-VBLAST } & ML & $\mathcal{O}\left(\left(M_{\text {VBLAST }}\right)^{N_{t} L}\right)$ & $N_{t}$ \\
\cline { 2 - 4 } & MMSE & $\mathcal{O}\left(L^{3} N_{r}^{3} N_{t} K\right)+\mathcal{O}\left(L^{3} N_{r} N_{t}^{3} K\right)+\mathcal{O}\left(L^{3} N_{r}^{2} N_{t}^{2} K\right)$ & $N_{t}$ \\
\hline \multirow{2}{*}{ CP-aided SC-VBLAST } & MMSE-FDE & $\mathcal{O}\left(N_{r} N_{t}^{2} K\right)+\mathcal{O}\left(N_{t}^{3} K\right)+\mathcal{O}\left(N_{t} K^{2}\right)+\mathcal{O}\left(N_{t}^{2} K M_{\text {VBLAST }}\right)$ & $N_{t}$ \\
\hline \multirow{2}{*}{ CP-aided OFDM-VBLAST } & ML & $\mathcal{O}\left(N_{r}\left(M_{\text {VBLAST }}\right)^{N_{t}} K\right)$ & $N_{t}$ \\
\cline { 2 - 4 } & MMSE & $\mathcal{O}\left(N_{t}^{2} N_{r} K\right)+\mathcal{O}\left(N_{t}^{3} K\right)$ & $N_{t}$ \\
\hline CP-aided OFDM-STBC & ML & $\mathcal{O}\left(N_{t} N_{r}^{2} K\right)$ & $N_{t}$ \\
\hline \multirow{2}{*}{ CP-aided SC-SM } & ML & $\mathcal{O}\left(\left(N_{t} M_{\mathrm{SM}}\right)^{K}\right)$ & 1 \\
\cline { 2 - 4 } & MMSE-FDE & $\mathcal{O}\left(N_{r} N_{t}^{2} K\right)+\mathcal{O}\left(N_{t}^{3} K\right)+\mathcal{O}\left(N_{t} K^{2}\right)+\mathcal{O}\left(N_{t}^{2} K M_{\mathrm{SM}}\right)$ & 1 \\
\hline CP-aided OFDM-SM & $\mathrm{MMSE}$ & $\mathcal{O}\left(N_{r} N_{t}^{2} K\right)+O\left(N_{t}^{3} K\right)$ & $N_{t}$ \\
\hline ZP-aided SC-SM & $\mathrm{ML}$ & $\mathcal{O}\left(\left(N_{t} M_{\mathrm{SM}}\right)^{L}\right)$ & 1 \\
\cline { 2 - 4 } & LSS & $\mathcal{O}\left(N_{t}^{2} M_{\mathrm{SM}} M K\right)+\mathcal{O}\left(L K N_{t} N_{r} M\right)$ & 1 \\
\hline
\end{tabular}

$M_{\text {VBLAST }}:$ the APM order used in VBLAST scheme.

$M_{\mathrm{SM}}$ : the APM order used in SM scheme.

$L:$ the length of the CIR.

$M$ : the selected $M$ value in the near-ML LSS detector.

$K$ : the length of the frame.

$N_{t}$ : the number of transmit antennas.

$N_{r}$ : the number of receive antennas.

option, further investigations are required to strike an attractive tradeoff amongst the detection complexity imposed, the attainable BER, as well as the achievable transmission rate and power efficiency.

\section{Some Transmitter Design Variants}

In recent years, diverse generalized SM schemes have been proposed, such as the GSM of [73]-[75], the SSK scheme of [37], and the GSSK arrangements of [76], [77]. However, these schemes have been predominantly investigated in the context of narrow-band scenarios, assuming that SM symbols are transmitted over flat-fading channels. However, in practice, most of the wireless channels exhibit frequency selective properties. This leads to several further generalized SM versions, which aim to provide increased transmission rate, improved the energy efficiency or higher multipath diversity gain [78][80]. Explicitly, there are three types of SC-SM variants: the SC-GSM scheme [78], the space and time-dispersion modulation (STdM) scheme [79] and the family of SFSK arrangements [80], which are detailed as follows:

- SC-GSM: As a natural extension of SM, the GSM scheme was proposed in [81] for the sake of achieving increased-rate data transmission, which activates several TAs - rather than a single TA or all TAs — to carry the information symbols during each time slot. Specifically, in GSM, $N_{g}$ out of $N_{t}\left(N_{g}<N_{t}\right)$ TAs are activated during each time slot, where the information bits are conveyed both by the active TA-index combinations as well by the $N_{g}$ conventional APM symbols [82]. This extension has also been incorporated into SC-based transmission over dispersive channels, where the single-RF restriction is relaxed by using $N_{g}$ RF chains [78]. In general, SC-GSM includes SC-SM as a special case associated with $N_{g}=1$, which strikes a trade-off among the transmit rate, the RF 476 cost as well as the detection complexity.

- STdM: By exploiting the principle of SM in both time- 478 dispersion and space dimensions, an STdM scheme was 479 proposed for frequency selective fading channels in [79], 480 where the channel's time-slot indices are exploited for 481 conveying additional information, apart from the active 482 TA index and the conventional APM symbols of SM. In 483 contrast to conventional SC-SM scheme, STdM exploits 484 the resolvable multipath components of the frequency- 485 selective channel as a novel means of conveying addi- 486 tional source information. Specifically, in the STdM 487 scheme, only one out of $N_{t}$ TAs was activated in one out 488 of $L-1$ resolvable multipath components, where $L$ is the 489 CIR length. In [79], the energy efficiency of STdM was 490 evaluated based on a realistic power consumption model 491 and it was found to be more energy-efficient than SC-SM. 492 Note that the design of STdM critically hinges on the ide- 493 alized assumption that independent symbol-spaced taps 494 are available for MIMO channels.

- SFSK: In [80], by intrinsically amalgamating the con- 496 cept of SM and linear dispersion codes (LDCs) [83], 497 Ngo et al. proposed the design philosophy of SFSK, 498 which selects one out of $\varphi$ orthogonal frequencies by a 499 frequency shift keying (FSK) modulator. In contrast to 500 the TA-index in conventional SM, in SFSK, the specific 501 indices of the pre-designed space-time dispersion matri- 502 ces are exploited for conveying extra implicit information. 503 Moreover, this concept was further extended in [80] to 504 enjoy benefits offered by the time, space and frequency 505 domains. SFSK may be viewed as an SC-based trans- 506 mission scheme, because only a single frequency tone 507 (out of multiple carriers) is utilized in each transmis- 508 sion. Note that the transmission of space-time dispersion 509 


\section{Detection techniques for SC-SM

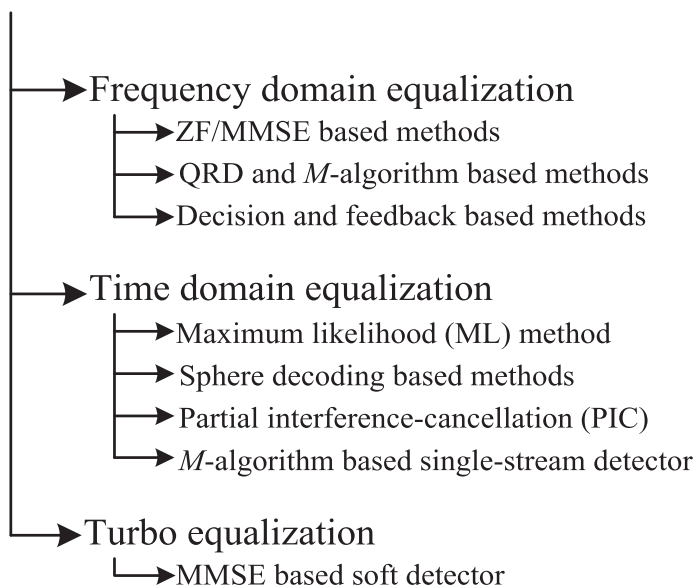

Fig. 6. Overview of SC-SM detectors.

matrix requires multiple $\mathrm{RF}$ chains and the advantages of single-RF are eroded, except for the low-rate designs of [2].

\section{E. Detector Design}

Traditional receiver architectures designed for conventional SM and classic MIMO schemes may not be directly suitable for the family of SC-SM, due to the following reasons:

(1) Conventional SM detectors of [19], [84], [85] are focused on the detection of the TA index and APM symbol in flatfading scenarios, which usually ignore the inter-symbol interference (ISI) caused by the channel's frequency selectivity.

(2) Most of the existing MIMO detectors conceived for multipath fading channels are proposed under the assumption that the MIMO channel matrix has column full-rank $\left(N_{r} \geq N_{t}\right)$ [58], [86]. However, an attractive advantage of $\mathrm{SM}$ is that it can efficiently operate also in the challenging scenario of asymmetric/unbalanced MIMO systems, whose channel matrices may be rank-deficient due to having more TAs than RAs.

This inspired recent research efforts in devising sophisticated receivers for SC-SM systems. At the time of writing, there are three typical receiver architectures: frequency-domain equalizer (FDE) [66], [87], time-domain equalizer (TDE) [70], [71], [88] and the powerful turbo equalizer (TEQ) [87]. A sketch of these detection techniques conceived for SC-SM systems is provided in Fig. 6. Next, they will be characterized in a little more detail.

1) Frequency-Domain Equalizer: An attractive lowcomplexity approach to ISI mitigation, in scenarios exhibiting a long CIR, affecting thousands of bits is constituted by FDEs, because we can rely on single-tap frequency-domain channel transfer factor (FDCHTF) instead of a time-domain equalizer having thousands of taps. Hence, SC systems using FDEs (SC-FDE) have been adopted in some recent communication standards, such as the third generation partnership project (3GPP) long-term evolution (LTE) standard. The benefits of SC-FDE also have been explored in the context of various
MIMO techniques. A detailed overview of SC-FDE techniques 548 was presented in [62], [89] and both the MMSE-based FDEs 549 and the decision-feedback equalizer (DFE) based FDEs were 550 introduced. As a novel MIMO technique, SM may also be 551 beneficially combined with SC-FDE methods for combating 552 the effects of ISI.

To this end, in [66] various state-of-the-art FDE algorithms 554 conceived for VBLAST were investigated in the context of CP- 555 aided SC-SM systems. Specifically, the zero forcing (ZF)-based 556 FDE of [90], the MMSE-based FDE (MMSE-FDE) of [91], and 557 the decision feedback based approaches [62] as well as the QR 558 decomposition combined with the $M$-algorithm (QRD- $M$ ) [62] 559 were utilized to recover the transmit vector, in which an SM sig- 560 nal vector was viewed as a special type of the VBLAST signal. 561 Then, the low-complexity matched filter (MF) based detec- 562 tion method of [16] was utilized, where the activated TA index 563 and the modulated APM constellation point are separately esti- 564 mated. That detector directly combines the classic VBLAST 565 detector conceived for ISI channels and the MF-based detector 566 of conventional SM schemes for demodulation. It was shown 567 in [66] that the QRD- $M$ detector outperforms other detectors in 568 the context of SC-SM systems, since it was designed based on 569 the near-optimal tree search principle by selecting only $M$ most 570 possible survived branches [92]. Moreover, in [87] the authors 571 exploited the MMSE criterion to derive the weights of the FDE 572 for the CP-aided SC-SM. Similar to the classic MMSE-FDE, 573 the received signals were first converted to their frequency- 574 domain versions and then MMSE-based linear filtering was 575 invoked for estimating the frequency-domain SC-SM signals 576 by minimizing the average minimum square error between the 577 frequency-domain signals and the estimates. Then, these esti- 578 mates were converted to their time-domain counterparts, which 579 were further divided into several independent SM symbols. 580 Finally, the single-stream symbol-based ML detector of [84] 581 was employed to each SM symbol for jointly detecting both 582 the active TA index as well as the transmitted APM symbol. In 583 contrast to the classic equalizers designed for traditional MIMO 584 systems, it was found in [87] that their MMSE-based FDE taps 585 depend on the sparsity of the SM symbols. In short, this detector 586 first employs a carefully designed MMSE-FDE for generating 587 an initial estimate and then invokes the conventional single- 588 stream ML detector of SM for symbol-by-symbol detection. As 589 shown in [87], the complexity of this detector is independent 590 of the CIR length. However, most of the above-mentioned FDE 591 algorithms are only suitable for scenarios, where the channel 592 matrix is of full-rank, i.e. $N_{r} \geq N_{t}$.

2) Time-Domain Equalizer: A well-known classic 594 approach to ISI-mitigation in the SC-based systems is 595 based on the employment of a TDE. Various TDE methods 596 [62], such as the ML-based TDE, the low-complexity linear 597 TDE, the parallel interference cancellation (PIC), as well as 598 the successive interference cancellation (SIC) have been exten- 599 sively studied in the context of MIMO systems. In contrast 600 to the transmitted signals generated by conventional MIMO 601 schemes, the transmit vectors of SM schemes are sparsely 602 populated, since typically only a single TA is activated [18], 603 [19]. This constraint makes SM rather different from the classic 604 STBC or the VBLAST schemes [1] and the TDE of SC-SM 605 
has to be carefully designed for exploiting the benefits of both $\mathrm{SC}$ and SM techniques.

In [70], an optimal ML detector was proposed for SC-SM schemes, which carries out an exhaustive search for finding the global optimum in the entire transmit signal space. This detector jointly detects the entire transmission frame to retrieve the original $m_{r}$ bits. The advantages of the ML detector over the $\mathrm{ZF}$ and the MMSE detectors were evaluated for the CP-aided SC-SM schemes. However, as both the transmission rate and $K$ increase, the complexity of the ML detector becomes excessive.

To attain a high diversity gain, in [71] a ZP-aided SC-SM scheme was investigated. To reduce its detection complexity, the authors viewed this system as a new kind of STBC scheme, which allows the use of STBC's generalized distribution law (GDL) to simplify the corresponding ML detector. It was found that the order of the ML detection complexity in the ZP-aided SC-SM scheme only depends on the CIR length rather than on the frame length $K$, as mentioned in Section II-B. To further reduce the complexity imposed by a large value of $L$, a low-complexity PIC-based receiver with SIC (PIC-R-SIC) was proposed in [71], which is capable of achieving both multipathand receiver- diversity gains (compared to ML detector) for some specific channel conditions. Compared to the conventional PIC-R-SIC designed for STBC schemes operating in a flat-fading scenario, the extended PIC-R-SIC detector is suitable for a dispersive channel, which operates by converting the dispersive multipath channel into a set of frequency-flat block fading subchannels.

Another promising method of reducing the complexity of the ML detector is constituted by the sphere decoding (SD) algorithms of [93], the concept of which is to search for the closest lattice points within a certain SNR-dependent search radius. However, the number of surviving search paths is still relatively high for a large tree and it may only be suitable for SC-SM schemes having a small frame length $K$.

Relying on the concept of the classic $M$-algorithm, in [88] the authors proposed a low-complexity single-stream (LSS) detector for avoiding the channel inversion operation of the PIC-R-SIC scheme, while striking a flexible tradeoff between the computational complexity imposed and the attainable BER. Note that in the traditional $M$-algorithm, the QR-decomposition requires the channel matrix to be a full-rank (column-wise) matrix. Hence, it can not be directly applied by the ZP-aided SC-SM, where the channel matrix may be rank deficient. In the proposed LSS, the QR-decomposition is avoided by properly exploiting the single-stream ML detection of [84]. It was found that their proposed LSS detector is also capable of efficient operation in the challenging rank-deficient channel scenarios.

3) Turbo Equalizer: It is worth noting that nearly all wireless communication systems employ some forms of forward error correction (FEC) to counteract unpredictable transmission errors, which require soft-decision based detectors rather than the hard-decision based TDE and FDE. The classic TEQ [94] has also been demonstrated to be an effective soft-decision receiver in frequency selective fading channels, incorporating both equalization and channel decoding. The basic concept of TEQ relies on the iterative exchange of soft information in the form of log-likelihood ratios (LLRs), between the equalizer and 664 the decoder. The trellis-based TEQ relying on the Viterbi algo- 665 rithm and the Bahl-Cocke-Jelinek-Raviv (BCJR) algorithm [94] 666 imposes high complexity in the context of SC-MIMO systems, 667 especially for high CIR lengths $L$ and for a large APM size 668 $M_{\text {SM }}$. Hence, the low-complexity yet efficient MMSE-based 669 TEQ is the one that is commonly used.

Recently, in [87] an MMSE based low-complexity soft- 671 decision FDE algorithm was developed for broadband high-rate 672 SC-SM systems, which is capable of operating in dispersive 673 channel having a long CIR. Specifically, they first proposed a 674 hard-decision aided SC-SM detector based on the MMSE crite- 675 rion and then developed its soft-decision version by employing 676 maximum a posteriori (MAP) demodulation. The MMSE-FDE 677 coefficients obtained are different from those derived for tra- 678 ditional MIMO schemes, because every SM symbol exhibits 679 sparsity. Moreover, in [87] a three-stage concatenated SC-SM 680 architecture was proposed for attaining a near-capacity perfor- 681 mance by amalgamating a recursive systematic convolutional 682 code and a unity-rate convolutional code, which was achieved 683 by exchanging the extrinsic information of these decoders a suf- 684 ficiently high number of times, until no more iteration gains 685 were achieved. Effectively, this resulted in a two-stage system. 686 Similar to the above-mentioned FDE detectors, this detector 687 was also designed under the constraint of $N_{r} \geq N_{t}$.

Note that most of the above-mentioned detectors assume 689 that perfect channel state information (CSI) is available at 690 the receiver. However, it is challenging to acquire accurate 691 CSI, which results in a severe degradation of the achievable 692 performance. This effect has been theoretically analyzed in nar- 693 rowband SM systems. In order to dispense with CSI-estimation, 694 some differential SM schemes have been proposed in flat- 695 fading scenarios [18]. However, these investigations have not 696 been extended to SC-SM. This issue will be further discussed 697 in Section V-F.

4) Performance: The observations above have shown that 699 the TDE-type detectors may be more attractive in the con- 700 text of SC-SM, since they are capable of retaining all the 701 benefits of SC and SM techniques. They are also suitable 702 for arbitrary antenna configurations, including the scenarios of 703 $N_{r} \leq N_{t}$. Fig. 7 characterizes the BER performance of the ZP- 704 aided SC-SM schemes in the context of $(2 \times 1)$ and $(2 \times 2) 705$ MIMO channels at a throughput of $m_{\mathrm{SM}}=4 \mathrm{bpcu}$ for transmis- 706 sion over Rayleigh multipath channels having a uniform power 707 delay profile [71]. To evaluate the performance gaps between 708 the low-complexity sub-optimal detectors and ML detector, we 709 first consider a small frame length $K$ in our simulations and 710 then adopt a higher value of $K$. To be specific, in Fig. 7, similar 711 to the setup of [71], the frame length is set to $K=4$ and the 712 8-PSK constellation is adopted, the CIR length is $L=3$ and 713 different TDE algorithms are considered. We also consider the 714 identical-throughput CP-aided OFDM-SM schemes of [16] as 715 benchmarkers.

Observe in Fig. 7 (a) that the LSS detector performs well in 717 rank-deficient $(2 \times 1)$-element MIMO channels, while the PIC- 718 R-SIC method suffers from a multipath diversity reduction. For 719 $(2 \times 2)$ MIMO channels, the LSS detector having $M=8$ out- 720 performs the PIC-R-SIC method and provides a signal-to-noise 721 
(a) $N_{r}=1$

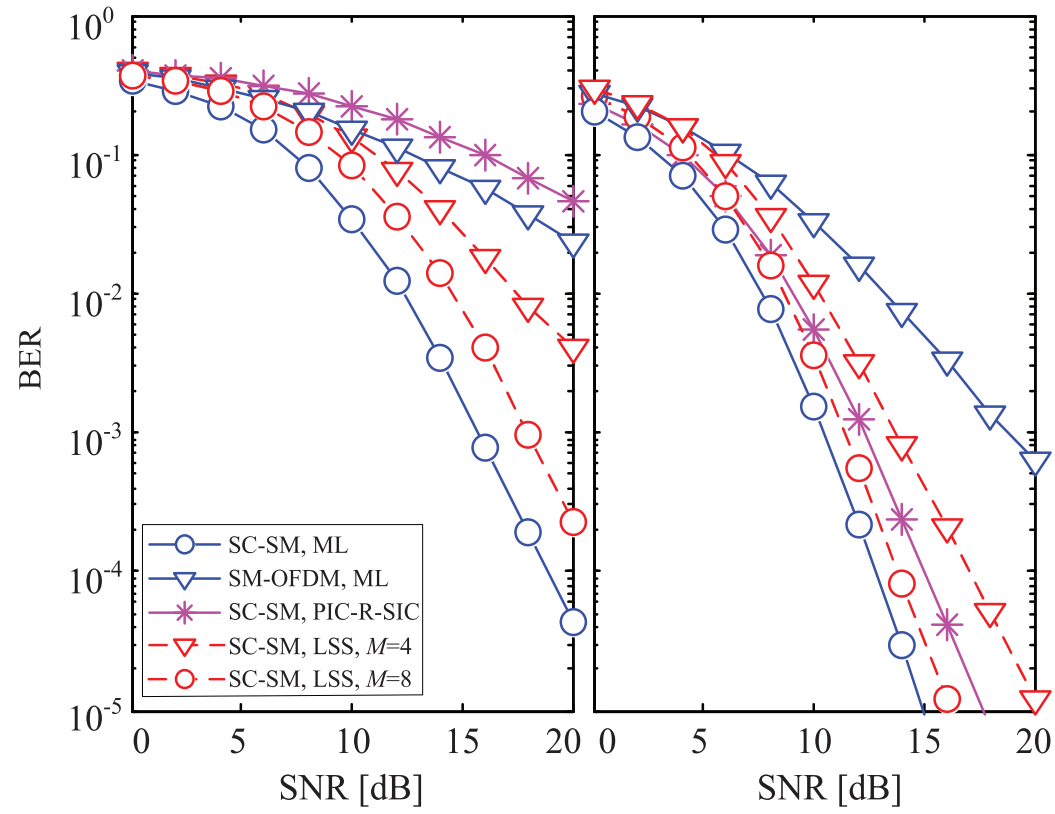

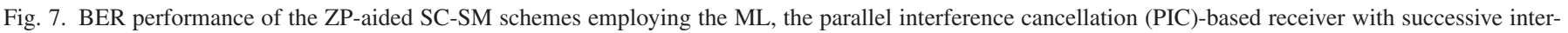

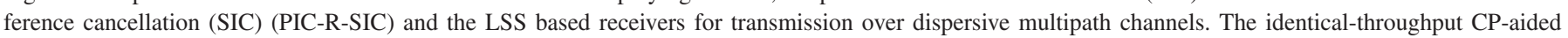

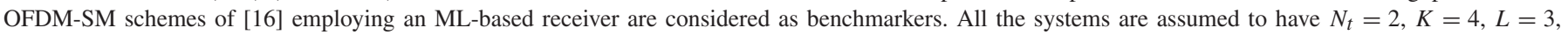
$m_{\mathrm{SM}}=4 \mathrm{bpcu}$ and using an 8-PSK constellation.

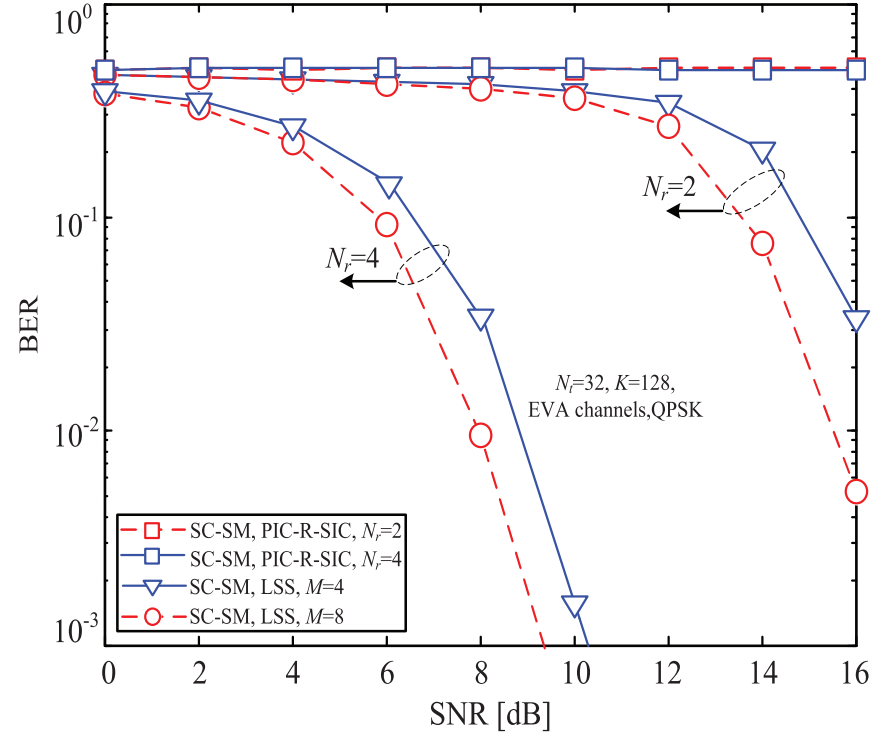

Fig. 8. BER performance of the ZP-aided SC-SM schemes employing the PICR-SIC and the LSS based methods for transmission over the EVA channels, having $L=6$, QPSK modulation and $N_{r}=2,4$. Here, the performance of these detectors with a higher number of TAs $N_{t}=32$ and a longer frame length $K=128$ are investigated, compared to Fig. 7 . ratio (SNR) gain of about $1.6 \mathrm{~dB}$ at the BER of $10^{-5}$. Moreover, in Fig. 7(b) the performance gap between the LSS detector and the exhaustive-search-based ML detector is only about $5 \mathrm{~dB}$ and $1 \mathrm{~dB}$ for $M=4$ and $M=8$, respectively. This gap can be further reduced by setting a larger value of $M$ at the cost of a higher complexity. In general, the LSS detector is capable of adjusting the parameter $M$ for striking a flexible tradeoff
TABLE III

COMPLEXITy ORders of DifFERENT TIME-DOMAIN DETECTORS FOR ZP-AIDED SC-SM SCHEME

\begin{tabular}{|l|l|}
\hline Detector & Complexity Order (Flops) \\
\hline ML & $\mathcal{O}\left(\left(N_{t} M_{\mathrm{SM}}\right)^{L}\right)$ \\
\hline PIC-R-SIC & $O\left(N_{t}^{2} K^{2} N_{r}\right)+O\left(K^{3} N_{t}\right)+O\left((K+L) N_{t}^{2} M_{\mathrm{SM}} K\right)$ \\
\hline LSS & $\mathcal{O}\left(N_{t}^{2} M_{\mathrm{SM}} M K\right)+\mathcal{O}\left(L K N_{t} N_{r} M\right)$ \\
\hline
\end{tabular}

between the attainable BER and the detection complexity 729 imposed.

The above-mentioned benefits of the LSS detector recorded 731 for the ZP-aided SC-SM are also visible in Fig. 8, where a 732 long frame length of $K=128$ and a large number of TAs, 733 namely $N_{t}=32$ are considered in the extended vehicular $a 734$ channel (EVA) model [90]. In Fig. 8, the BER performance of 735 the optimal ML detector is not provided due to its excessive 736 complexity. It is shown in Fig. 8 that the BER of the PIC-R- 737 SIC scheme may have an error floor effect under rank-deficient 738 channel conditions, which was also observed in [88]. $\quad 739$

5) Complexity: In Table III, the complexity orders of the 740 PIC-R-SIC, the ML and the LSS detectors employed in Figs. 7741 and 8 are compared. It is shown that the complexity of the ML 742 detector is unaffordable as it grows exponentially with the CIR 743 length $L$. Based on the results of Tables II and III, it is noted 744 that the time-domain based LSS detector has a similar com- 745 plexity order to that of the classic linear MMSE based detector. 746 In Tables IV and V, we also provide the approximate complex- 747 ity of the configurations adopted in Figs. 7 and 8. It is shown 748 that the LSS detector is capable of striking a flexible tradeoff 749 in terms of the BER attained and the complexity imposed by 750 adjusting the parameter $M$. 
TABLE IV

COMPleXity OF DifFERENT DETECTORS FOR ZP-AIDED SC-SM SCHEME IN TERMS OF THE NUMBER OF REAL-VALUED Multiplications, Where the Setup is the Same as That in Fig. 7

\begin{tabular}{|c|c|c|c|c|}
\hline \multicolumn{4}{|c|}{$N_{t}=2, K=4, L=3,8$-PSK } \\
\hline \multirow{2}{*}{ Detector } & \multirow{2}{*}{$\mathrm{ML}$} & \multirow{2}{*}{ PIC-R-SIC } & \multicolumn{2}{|c|}{ LSS } \\
\cline { 3 - 5 } & & & $M=4$ & $M=8$ \\
\hline$N_{r}=1$ & $1.3 \times 10^{7}$ & $1.0 \times 10^{4}$ & $5.4 \times 10^{3}$ & $1.1 \times 10^{4}$ \\
\hline$N_{r}=2$ & $2.7 \times 10^{7}$ & $2.5 \times 10^{4}$ & $1.1 \times 10^{4}$ & $2.1 \times 10^{4}$ \\
\hline
\end{tabular}

TABLE V

COMPlEXITY OF THE PIC-R-SIC AND LSS DETECTORS FOR ZP-AIDED SC-SM Scheme, Where the Setup is the Same as That IN Fig. 8

\begin{tabular}{|c|c|c|c|}
\hline \multicolumn{3}{|c|}{$N_{t}=32, K=128, L=6$, QPSK } \\
\hline \hline \multirow{2}{*}{ Detector } & PIC- & \multicolumn{2}{|c|}{ LSS } \\
\cline { 3 - 4 } & R-SIC & $M=4$ & $M=8$ \\
\hline$N_{r}=2$ & $5.9 \times 10^{12}$ & $1.4 \times 10^{9}$ & $2.2 \times 10^{9}$ \\
\hline$N_{r}=4$ & $7.6 \times 10^{12}$ & $2.2 \times 10^{9}$ & $4.3 \times 10^{9}$ \\
\hline
\end{tabular}

\section{Potential Advantages and Dis advantages}

In light of the transceiver design described in Section II, we summarize some potential advantages, tradeoffs and disadvantages of SC-SM in Fig. 9.

\section{A. Potential Advantages}

1) Simple Transmitter Design: In conventional MIMO systems, such as the VBLAST and the STBC schemes, the hardware complexity and cost rise with the number of TAs $N_{t}$. Moreover, the deleterious effects of RF circuit mismatches and coupling impairments also grow with the value of $N_{t}$. These factors limit the number of antennas. As shown in Fig. 2, SCSM relies on the unique encoding philosophy of activating one out of numerous TAs at each time slot, hence only a single RF chain $\left(N_{g}\right.$ RF chains for the SC-GSM scheme, $\left.N_{g}<N_{t}\right)$ is required instead of $N_{t}$ parallel RF chains. Hence its RF section has a reduced complexity and cost. On the other hand, in conventional OFDM-based MIMO transmissions, the complexity imposed by performing the DFT operations for its signal modulation and demodulation also increases with $N_{t}$. As shown in Section II and Fig. 2, this computational complexity and the RF hardware complexity can be reduced by employing the SC-SM based transmitter advocated.

2) Simple Receiver Design: Data detection in the conventional MIMO systems is one of the most challenging tasks in terms of both its computational complexity and its power consumption, especially in the LS-MIMO scenarios. In dispersive channels, the employment of SC or OFDM techniques further increases the dimensionality of the underlying detection problem. In SM, since only a single TA is activated for transmission, this allows us to design a low-complexity single-stream receiver, such as the LSS detector. Moreover, based on the unique bit-to-vector mapping rule of SC-SM detailed in Algorithm 1, the transmit vector of SC-SM associated with each TA has as low a fraction of nonzero elements as $K / N_{t}$ for each row of $\mathbf{X}$. Usually, a matrix or vector is referred to as sparse, if the number of nonzero elements in it is less than $20 \%$ of the total number of elements. Since the sparsity ratio of SC-SM is $1 / N_{t}$, for $N_{t}>4$ the transmit matrix $\mathbf{X}$ is deemed sparse and hence the detection can be regarded as a 790 sparse reconstruction problem. Therefore, the classic sparse 791 reconstruction theory [95], [96] may be invoked for recovering 792 the transmitted signals.

Accordingly, a number of related contributions have con- 794 centrated on the design of SM detection schemes based on 795 compressive sensing (CS). A message passing detection algo- 796 rithm was proposed in [105] for the multiple access channel 797 (MAC) in conjunction with a high number of antennas at the 798 BS. An iterative detector was developed for large-scale MACs 799 in [106], where the authors decoupled the antenna and symbol 800 estimation processes for reducing the total detection complex- 801 ity. The algorithm introduced in [112] beneficially exploits the 802 sparsity of SM transmission in the MAC. In [107], a general- 803 ized approximate message passing detector was preferred to the 804 high-complexity stage-wise linear detector. A closely related 805 approach has also been developed in [113] in order to miti- 806 gate the effects of spatial correlation imposed on tightly-packed 807 antennas.

3) Low PAPR and Robustness to Phase Noise: OFDM has 809 attracted substantial interests because it offers a powerful and 810 practical means to mitigate the effects of ISI in high-throughput 811 MIMO transmissions [58]. However, the DFT operation based 812 modulation at the transmitter disperses each sub-carrier's mod- 813 ulated signal across the entire DFT-block and hence erodes the 814 single-RF benefits of SM-MIMO [87], whilst simultaneously 815 resulting in a high PAPR. By contrast, SC-SM is capable of 816 retaining all the benefits of SM, whilst exhibiting a lower PAPR 817 than its OFDM-based counterpart [66], [71], [87]. As a result, 818 the performance of SM is less affected by the transmitter's 819 power amplifier nonlinearities. A further benefit of SC-SM is its 820 higher robustness both to the frequency offset and to the phase 821 noise, than that of the OFDM scheme, owing to its inherent SC 822 structure.

As an example, we assume that the number of TA is $N_{t}=2, \quad 824$ the throughput is $m_{\mathrm{SM}}=5 \mathrm{bpcu}$. The data block size is $K=825$ 256 and the oversampling factor is set to $\beta=4$ for PAPR cal- 826 culation as pulse shaping is considered. In Fig. 10, we present 827 the transmit signal generation and PAPR calculation process of 828 SC-SM and OFDM-SM schemes. The detailed signal genera- 829 tion processes of SC-SM and OFDM-SM are similar to that 830 in Fig. 4, except that a pulse shaping unit is added in SC-SM 831 scheme for practical signal transmission.

Based on the transmit signal model of Fig. 10, the cor- 833 responding complementary cumulative distribution functions 834 (CCDFs) of the PAPR of both SC-SM and OFDM-SM systems 835 are shown in Fig. 11. For the sake of simplicity, we only give 836 the CCDF result of the PAPR for the signal vector transmitted 837 over TA 1 , since the CCDF result recorded at TA 2 is similar 838 to that of TA 1 . The CCDF explicitly shows the probability of 839 having a PAPR, which is higher than a certain PAPR threshold 840 of $\mathrm{PAPR}_{0}$, namely that we have $\operatorname{Pr}\left\{\mathrm{PAPR}>\mathrm{PAPR}_{0}\right\}$. To eval- 841 uate the effects of pulse shaping on SC-SM, we convolve each 842 transmitted symbol waveform with a raised-cosine filter having 843 the roll-off factor $\alpha$.

As observed in Fig. 11, in the absence of pulse shaping, the 845 PAPR of the SC-SM scheme is about $8.5 \mathrm{~dB}$ lower than that 846 of the SC-OFDM scheme at a CCDF of $10^{-3}$. As a further 847 


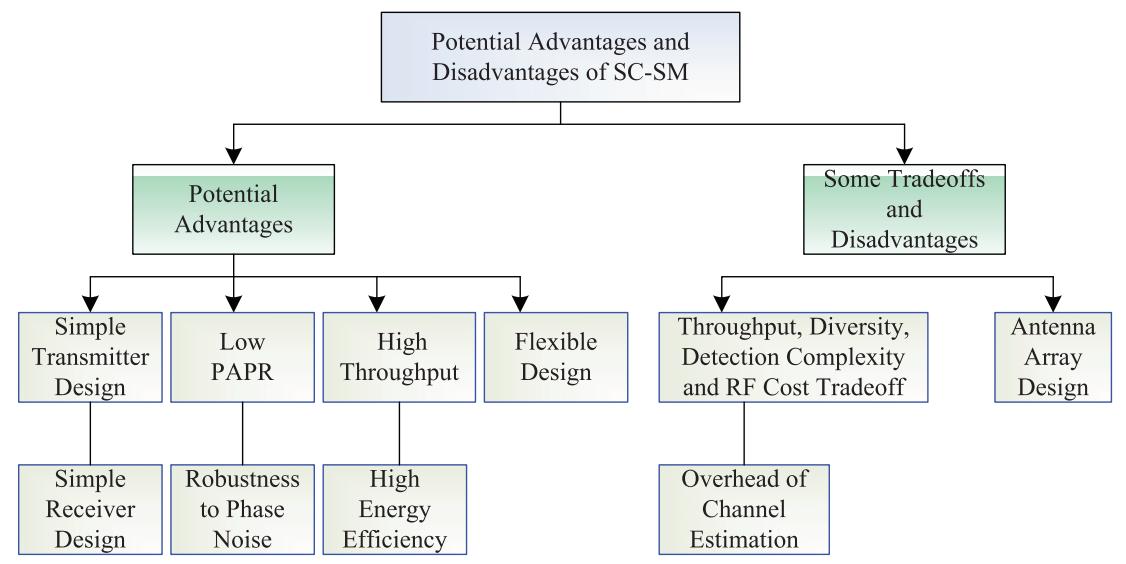

Fig. 9. A summary of main advantages and disadvantages of the SC-SM scheme.

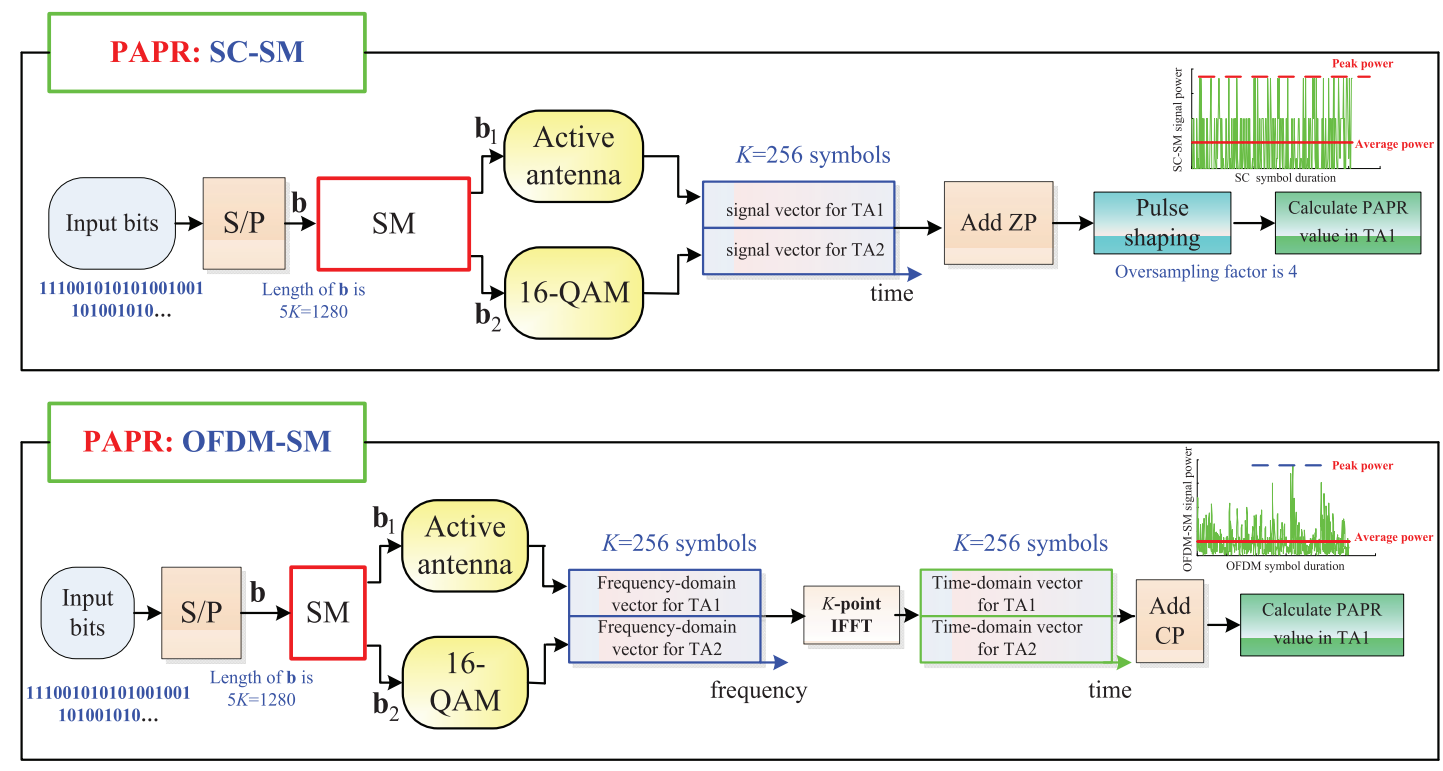

Fig. 10. The example of PAPR calculations for SC-SM and OFDM-SM schemes with $N_{t}=2, m_{\mathrm{SM}}=5$ bits/channel-use. We use 4 times oversampling to calculate PAPR for each block when pulse shaping is considered.

metric, the PAPR of the transmitted SC-SM signals only has a $1 \%$ probability of being above $3.5 \mathrm{~dB}$. Fig. 11 also shows that in conjunction with the raised-cosine filter, the PAPR increases for the SC-SM schemes, but it still remains better than that of OFDM-SM. It is observed in Fig. 11 that the performance can be improved by using a larger value of the roll-off factor $\alpha$ at the cost of an increased out-of-band radiation [82]. This implies that there is a tradeoff between the attainable PAPR and the out-of-band radiation imposed.

In Fig. 11, the effects of antenna switching in SM are not considered in the design of the pulse shaping filter. As shown in [117], a high roll-off factor is necessary for conventional raisedcosine filter to ensure that the transmit power is concentrated within a short time period so as to enable a single RF chain in SM. Hence, two large values of $\alpha$ are utilized in the PAPR comparison of Fig. 11, such as $\alpha=0.6$ and 0.8 . The design of time-limited waveforms for single-RF based SC-SM scheme is still a challenge, which will be further discussed in Section V-A.

4) High Throughput and High Energy Efficiency: As a new three-dimensional (3-D) hybrid modulation scheme, SM exploits the indices of the TAs as an additional dimension 868 invoked for transmitting information, apart from the classic 869 two-dimensional (2-D) APM [82]. SC-SM also adopts this 870 principle and hence achieves a higher throughput than that 871 of the single-antenna and the STBC-MIMO systems. In dis- 872 persive channels this gain can be further exploited by using 873 the STdM-like schemes of [79], which relies on the index of 874 the resolvable multipath links as a means of conveying addi- 875 tional source information. Since SM can be realized by using 876 a single RF front-end, the high-cost power amplifier, which is 877 typically responsible for the vast majority of power dissipation 878 at the transmitter, can be reduced. Specifically, recent results 879 based on a realistic power consumption model have shown that 880 a SM-aided base station (BS) has a considerable power con- 881 sumption gain compared to the multi-RF chain assisted MIMO 882 arrangements (up to $67 \%$ more energy efficient in the context of 883 $N_{t}=4$ ) [97]. This energy benefit may also be retained by the 884 SC-SM systems.

5) Flexible Design: As shown in Fig. 2, due to the single- 886 TA transmission mode, the minimum number of RAs required 887 


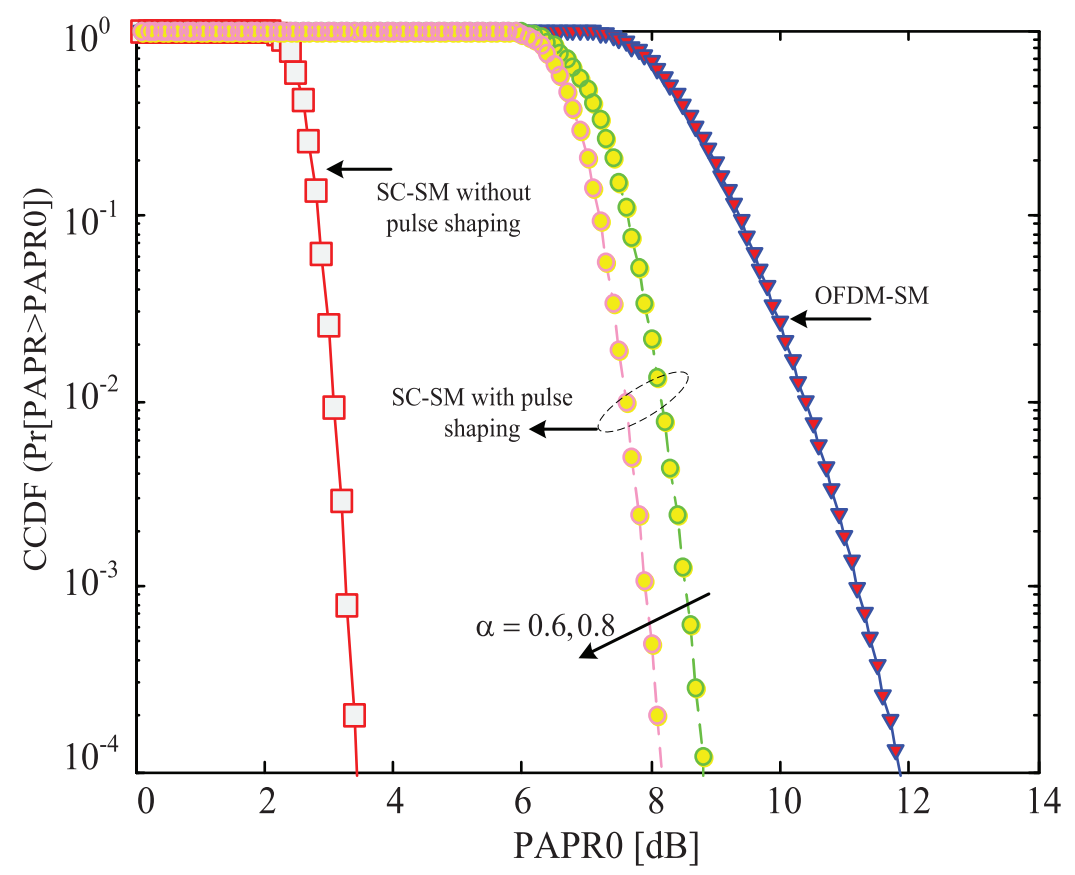

Fig. 11. Comparison of CCDF of PAPR for the SC-SM and the OFDM-SM systems having $N_{t}=2, K=256, m_{\mathrm{SM}}=5$ bpcu and operating with 16-QAM constellation. For the SC-SM with pulse shaping in the classic signal domain, the roll-off factor of the raised-cosine pulse filter is $\alpha=0.6$ and 0.8 .

for efficient detection can be set to one, regardless of how many TA there are. Hence, SC-SM can be flexibly configured for diverse TA and RA configurations, especially for the challenging scenario of asymmetric/unbalanced MIMO systems. As a further advance, SC-SM can be jointly designed in combination with the classic VBLAST and the STBC schemes for striking a flexible tradeoff among the attainable transmit rate, the diversity gain and the cost, which retains the key advantages of SM, while activating multiple TAs [75], [98]. Furthermore, SC-SM can be employed for exploiting the potential multipath diversity or the resolvable multipath-component-based multiplexing gain available in dispersive channels, i.e., the ZP-aided SC-SM scheme of [71] and the STdM scheme of [79]. Additionally, in SC-SM, the prefix vector can be flexibly selected according to the practical system requirements.

\section{B. Some Tradeoffs and Disadvantages}

1) Throughput, Diversity, Detection Complexity, and $R F$ Cost Tradeoff: In contrast to V-BLAST which is capable of achieving a high multiplexing gain, the family of SC-SM schemes may offer only a logarithmic (rather than a linear) increase of the throughput with the number of TA $N_{t}$ or the number of TA combinations. This may limit SC-SM for achieving very high spectral efficiencies. Moreover, the conventional SC-SM schemes only achieve receiver diversity and multipath diversity, but no transmit diversity. In conventional narrowband SM scheme, this impediment can be circumvented by employing open-loop as well as closed-loop transmit symbol design techniques [16]. However, these techniques may be not directly suitable for SC-SM systems. Indeed, the degree of freedom in a MIMO system can be allocated by different ways to achieve different tradeoff among different conflicting performance fac- 918 tors. As a new MIMO scheme, SC-SM exploits this degree 919 of freedom to reduce the number of transmit RF chains and 920 the detection complexity, at certain losses of diversity and 921 multiplexing gains.

2) Overhead of Channel Estimation: The coherent detec- 923 tion of the SC-SM scheme in Fig. 2 requires that CSI is 924 available at the receiver. However, it is challenging to acquire 925 accurate CSI of all MIMO channels. The peculiar transmit 926 encoding of SC-SM based over a limited number of RF chains 927 may introduce an additional training overhead, since the CSI of 928 the MIMO subchannels may not be estimated simultaneously. 929

3) Antenna Array Design: Compared to the conventional 930 full-RF based MIMO schemes, the configuration and deploy- 931 ment of the TA arrays for the single-RF based SC-SM pose 932 some design challenges, because it requires fast TA switching 933 and low spatial correlation between TAs. Due to the unique 934 encoding principle of SC-SM, the active TA index used to 935 convey information may be changed symbol-by-symbol, hence 936 a high-speed RF switching is required. Moreover, SC-SM 937 requires sufficiently low correlation among the channels of the 938 spatial streams for yielding adequate BER performance. Hence, 939 the channel correlation and mutual coupling effects should be 940 carefully considered in the TA array design and implementation 941 for SC-SM based systems. 942

These above-mentioned advantages and disadvantages indi- 943 cate that the SC-SM scheme appears to be an attractive 944 low-complexity low-cost option for the emerging family of LS- 945 MIMO systems. Recently, many promising preliminary solu- 946 tions have been developed to circumvent the above-mentioned 947 disadvantages, which succeeded in improving the tradeoff 948 among the conflicting factor associated with the design of SC- 949 SM systems, such as the computational complexity imposed, 950 
the attainable BER, the achievable throughput, the RF cost and the pilot overhead, just to name a few. For example, the SCGSM scheme may be invoked for improving the throughput, and the STBC scheme can be amalgamated with SC-SM for the sake of increasing the transmit diversity order, while differential SC-SM schemes can be developed for reducing or eliminating the pilot overhead. More related discussions will be provided in Section V. In the next Section, we will provide an up-to-date review of the application of SC-SM in LS-MIMO scenarios.

\section{SC-SM FOR MUltiUser LS-MIMO SySTEMS}

The observations and advantages described above have recently sparked off a flurry of research activities aimed at understanding the system design, the signal processing and the detector concepts of SC-SM for conceived multiuser (MU) LSMIMO systems, as shown in Table VI. In this section, we first review and discuss the SC-based transmission research in the context of LS-MIMO design and then focus our attention on the recent SC-SM schemes proposed for the LS-MIMO transmissions.

\section{A. SC-Based Transmission for LS-MIMO Systems}

Again, in the design of LS-MIMO systems, most of the existing contributions rely on the assumption of flat fading channels. One of the reasons for this assumption is that there is some evidence that ISI can be treated as additional thermal noise [10], [65]. However, this assumption has a limited applicability for practical transmissions over dispersive channels, when only a limited number of TAs is available. For the sake of combating the effects of ISI introduced by propagation over frequencyselective fading channels, OFDM is an attractive technique, since it facilities single-tap based equalization. However, it suffers from a high PAPR, as shown in Section III.

For these reasons, various LS-MIMO designs have been investigated in the context of energy-efficient SC or SC-like schemes [12], [63]-[65]. More specifically, in [13], a new softoutput data detector was proposed for uplink transmission by employing SC frequency division multiple access (SC-FDMA)based LS-MIMO systems. In [13] some practical implementations were conceived for the scenario where all users are equipped with a single antenna. Specifically, the complexity issues of data detection were addressed and a low-complexity soft MMSE-based detector was proposed in [13]. In particular, the inherent matrix inversion of the MMSE algorithm was approximated by a low-complexity Neumann series expansion. It was shown that their proposed method imposes a complexity order of $\mathcal{O}\left(U^{2}\right)$, which is lower than the complexity order of $\mathcal{O}\left(U^{3}\right)$ imposed by the conventional MMSE-based detector, where $U$ is the number of users. However, this approximate method may result in a degradation of BER performance.

In [63] the authors proposed a novel SC-based LS-MIMO scheme for frequency-selective multiuser Gaussian broadcast channels, where efficient downlink precoding was invoked. This transmit precoder (TPC) design is based on the assumption that the number of TAs $N_{\text {tot }}$ at the BS is substantially higher than the number of users $U\left(N_{t o t}>>U\right)$. Hence the channel hardening effect can be exploited to design the TPC matrix and 1005 the resultant TPC matrix is the linear weighted channel matrix 1006 (matched filter), instead of the inversion of the channel matrix. 1007 The extent of channel hardening can be viewed a measure of 1008 channel orthogonality, as discussed in [7]-[12]. It was shown 1009 [63] that the proposed SC scheme achieved a near-optimal 1010 sum-rate, with the aid of a low-complexity equalization-free 1011 receiver.

1012

More recently, in [64], the symbol-error rates (SERs) of the 1013 MF and MMSE detectors were investigated in the context of 1014 large-scale multiple-input single-output (MISO) systems both 1015 for a negative-exponentially decaying CIR model as well as for 1016 the typical urban channel model [99]. It was found [64] that the 1017 MMSE based detector performs better than the MF receiver. In 1018 [65], the authors investigated the ML equalization of the SC- 1019 based LS-MIMO uplink for transmission over a Rician fading 1020 channel, in order to mitigate the ISI generated by the combina- 1021 tion of the signals received from different antennas through a 1022 low-complexity MF. Moreover, by using this novel design, the 1023 MU-interference (MUI) caused by the line-of-sight (LOS) com- 1024 ponents, which may lead to an error-floor in the high SNR 1025 region for the conventional single-tap equalizer of [64], can be 1026 effectively mitigated.

Note that although the above-mentioned research shows that 1028 the SC-based transmission constitutes a promising design alter- 1029 ative for LS-MIMO transmissions, most of the existing research 1030 has been based on the assumption that $N_{\text {tot }}>U$ is satis- 1031 fied and that all users are equipped with a single antenna. The 1032 existing solutions may be further improved in order to meet 1033 the demand for high-throughput transmissions, in which all the 1034 users are equipped with multiple antennas and the number of 1035 users $U$ is large. In this case, future SC-based MIMO transmis- 1036 sion technique should be designed to satisfy a diverse range 1037 of practical requirements and to strike an attractive tradeoff 1038 amongst the RF cost, the detection complexity and the BER 1039 performance. As it will be shown in the following two subsec- 1040 tions, SC-SM can indeed be further developed for designing 1041 LS-MIMO systems to achieve these benefits both in downlink 1042 and uplink transmissions.

1043

\section{B. SC-SM Large-Scale MIMO Designs for Downlink 1044 Transmission

Owing to the compelling advantages of the SC-SM scheme 1046 discussed in Section III, recently it has been proposed for 1047 the MU LS-MIMO downlink, where the base station may be 1048 equipped with hundreds of TAs but only a few RF chains, while 1049 each user's receiver can be equipped with either a single or mul- 1050 tiple antennas yet relying on a single RF chain. The general 1051 downlink model of the multiuser SM based LS-MIMO system 1052 is shown in Fig. 12 (a). One of the key design challenges of this 1053 architecture is to construct a beneficial TPC for mitigating the 1054 MUI.

To this end, in [100] a single-cell downlink MU SM broad- 1056 cast framework was investigated, which has $N_{\text {tot }}$ TAs at the base 1057 station and $U$ active users, while each user is equipped with a 1058 single RA. In the proposed scheme, the $N_{\text {tot }}$ TAs are split into 1059 $U$ subsets each associated with $N_{d}$ TAs and each subset is then 1060 
TABLE VI

Major Contributions on SC-SM And Its LaRge-SCALE Mimo Developments

\begin{tabular}{|c|c|c|}
\hline Year & Author(s) & Contribution \\
\hline 2008 & Mesleh et al. [16] & The general bits-to-symbol mapping model of SM was proposed. \\
\hline \multirow[t]{2}{*}{2011} & Ngo et al. $[80]$ & $\begin{array}{l}\text { The philosophy of SFSK was proposed, which is a frequency-domain extension of SM } \\
\text { for dispersive channels. }\end{array}$ \\
\hline & Zhou et al. [66] & $\begin{array}{l}\text { The concept of SC-SM was proposed for dispersive channels and various linear and } \\
\text { nonlinear FDE methods were investigated. }\end{array}$ \\
\hline 2012 & Som and Chockalingam [70] & A diversity analysis framework was proposed for the CP-aided SC-SM. \\
\hline 2013 & Rajashekar et al. [71] & $\begin{array}{l}\text { A ZP-aided SC-SM system was proposed, which is capable of offering full multipath } \\
\text { diversity by employing a low-complexity PIC-R-SIC detector. }\end{array}$ \\
\hline \multirow[t]{5}{*}{2014} & Narayanan et al. [100] & A single-cell downlink broadcast MU SM framework was investigated. \\
\hline & Humadi et al. [101] & $\begin{array}{l}\text { A new SM-based transmitter was proposed for large-scale MU downlink transmission } \\
\text { systems, where can be viewed an extension of the RSM scheme for MU transmissions. }\end{array}$ \\
\hline & Narasimhan et al. [105] & $\begin{array}{l}\text { The superiority of the large-scale SC-SM over the conventional LS-MIMO was } \\
\text { investigated and two low-complexity MU detectors were proposed for uplink transmissions. }\end{array}$ \\
\hline & Wang et al. $[112]$ & $\begin{array}{l}\text { The sparsity and the prior probability distribution of the transmit signal were exploited } \\
\text { in the detector design of large-scale CP-aided SC-SM uplink transmission systems. }\end{array}$ \\
\hline & Sugiura and Hanzo [87] & $\begin{array}{l}\text { An MMSE based low-complexity soft-decision FDE algorithm was proposed } \\
\text { for the broadband CP-aided SC-SM systems. }\end{array}$ \\
\hline \multirow[t]{4}{*}{2015} & Narasimhan et al. $[78]$ & $\begin{array}{l}\text { A generalized SC-SM, termed as SC-GSM, was proposed for the LS-MIMO uplink } \\
\text { transmission systems, which is capable of striking a trade-off among the transmit rate, } \\
\text { the RF cost and the detection complexity. }\end{array}$ \\
\hline & Zheng et al. [79] & $\begin{array}{l}\text { An STdM scheme was proposed for frequency selective fading channels, where the channel } \\
\text { time-slot index was employed for conveying additional information. }\end{array}$ \\
\hline & Xiao et al. $[88]$ & $\begin{array}{l}\text { An attractive LSS detector was proposed for the ZP-aided SC-SM, which can efficiently } \\
\text { work in the rank-deficient LS-MIMO channels. }\end{array}$ \\
\hline & Wang et al. [113] & $\begin{array}{l}\text { The effects of the low-resolution analog-to-digital convertors (ADCs) were investigated in } \\
\text { large-scale CP-aided SC-SM uplink transmissions. }\end{array}$ \\
\hline
\end{tabular}

(a) Downlink
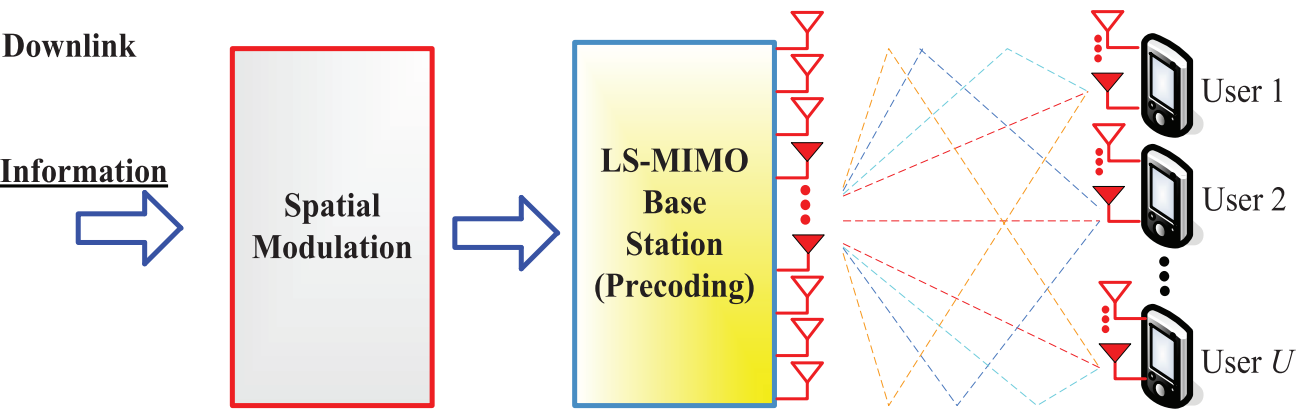

(b) Uplink
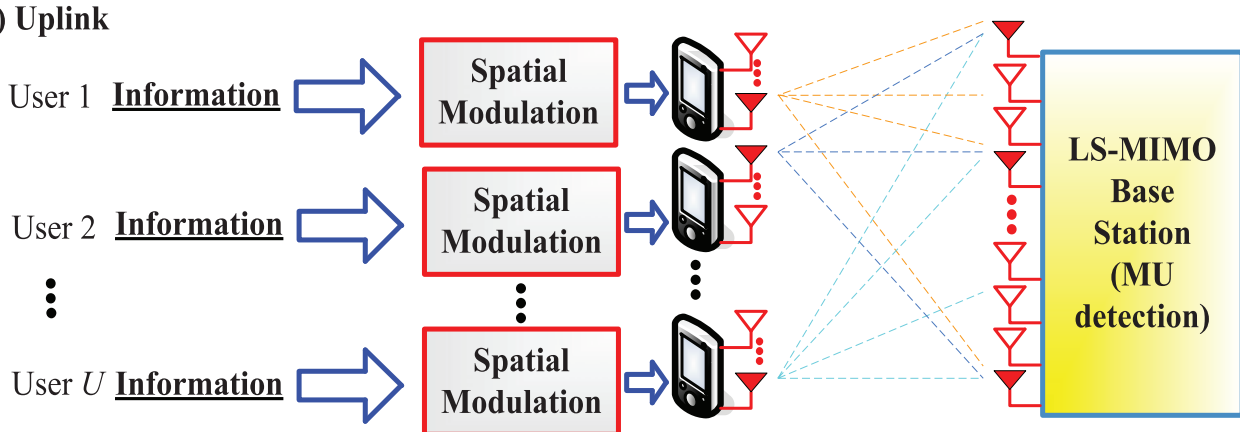

Fig. 12. The design framework for large-scale SC-SM based MU transmission. (a) The general downlink system model. (b) The general uplink system model.

allocated to a specific active user. These TA-subsets are then used for an SM-based transmission. Since there is a total of $U$ TA subsets and only a single TA is active in each subset, the number of RF chains required is equal to $U$. In [100], the 1064 SM-based data vector was preprocessed by using a carefully 1065 designed TPC for mitigating the MUI, while simultaneously 1066 
1067 retaining the benefits of SM. As shown in [100], the classic ZF 1068 and MMSE TPCs derived for conventional MU MIMO systems 1069 are not suitable for SC-SM based MU downlink systems. This is 1070 because SM enables data decoding by exploiting the differences 1071 among the CIRs of the transmit-to-receive wireless links, but 1072 these TPCs may eliminate the channel matrix and hence jeop1073 ardize the bits carried by the TA indices. The TPC designed in 1074 [100] has considered this effect, which is capable of eliminating 1075 of MUI and retaining the CIR differences. The resultant TPC 1076 depends on the MU channel matrix $\mathbf{H}_{\mathrm{MU}}$ and can be expressed 1077 as $\left(\mathbf{H}_{\mathrm{MU}}\right)^{-1} \operatorname{diag}\left(\mathbf{H}_{\mathrm{MU}}\right)$, where $\operatorname{diag}(\cdot)$ denotes the diagonal 1078 matrix operator.

1079

The simulation results in [100] shows that their proposed scheme is capable of providing the same BER performance as that of the single-user SM transmission, i.e., interference-free. To avoid CSI estimation, an interference-aware detector was also proposed in [100]. Unlike the conventional MIMO-aided MU downlink systems, [100] shows that the direct multiuser detection is not preferred for SC-SM based MU systems. An alternative method is their proposed TPC scheme with single-user detection.

Relying on a novel approach, in [101] the authors proposed a new SM-based transmitter for MU LS-MIMO systems, where the index of the active RA of each user was exploited to convey extra useful information. This scheme can be viewed as an extension of the point-to-point receiver-side SM (RSM) concept of [102]-[104] to MU scenarios. To be specific, in RSM, a particular subset of receive antennas is activated and the specific activated pattern itself conveys useful but implicit information [102]-[104]. In RSM, the classic ZF or MMSE-based TPC schemes can be used for ensuring that no energy leaks into the inactive RA patterns. Based on a similar TPC design principle to that of RSM, a pair of novel methods, termed as subchannel selection and zero-padding, were proposed in [101] for implementing this new SM-based transmitter in the context of MU LS-MIMO downlink systems, where the ZF-based TPC design criterion was used. Moreover, asymptotically tight upper bounds were derived for the average bit error probability (ABEP) of these methods and the simulation results validated that the zero-padding method is more robust to the MUI [101].

Table VII shows the comparisons of a range of potential SCSM designs at a glance. We can see that the existing SM-based LS-MIMO downlink transmission schemes have been focused on the TPC designs conceived only for narrow-band scenarios. How to extend these TPCs to broadband transmission is hence an important open issue for future research. A range of open design issues shown in Table VII will be discussed in more detail in Section $\mathrm{V}$.

\section{SC-SM Large-Scale MIMO Designs for Uplink} Transmission

More recently, SC-SM has also been developed for the uplink of multi-access scenarios and the general system model of the associated uplink SC-SM LS-MIMO system is shown in Fig. 12 (b). Compared to the downlink transmission, the key technological issue in Fig. 12 (b) becomes the design of lowcomplexity near-optimal detectors for the high-dimensional detection problems faced by the BS equipped with a large 1123 number of RAs.

To this end, in [105] the authors proposed a pair of low- 1125 complexity detection algorithms for SC-SM based LS-MIMO 1126 schemes operating in frequency flat-fading channels, supported 1127 by the graph-based message passing (MP) algorithm and the 1128 lattice-based local search concepts, respectively. Note that the 1129 graph-based MP algorithm is an attractive low-complexity near- 1130 optimal approach conceived for large search spaces [108], 1131 which has been used for the decoding of turbo codes [109], the 1132 detection of MIMO signals [110] as well as for data clustering 1133 [111]. The basic idea of the graph-based MP is to graphi- 1134 cally represent the factorization of a function and to compute 1135 marginals by passing messages over the edges of the graph 1136 [108]. The evolved version of the algorithm is mainly depen- 1137 dent on the associated graph structure and on the specific 1138 message passing method utilized. In [105], the MU SC-SM 1139 LS-MIMO system is represented as a factor graph, where the 1140 received symbols were viewed as the observation nodes, while 1141 the transmit symbols were viewed as the variable nodes. The 1142 messages passed between variable nodes and observation nodes 1143 in the factor graph are approximated by a Gaussian distribution, 1144 which will be detailed both in the example of Section IV-D and 1145 in Fig. 13.

1146

Moreover, in [105] the relationship of the large-scale SC- 1147 SM and of the conventional LS-MIMO arrangement was 1148 investigated and the numerical results demonstrated that the 1149 SC-SM schemes are capable of providing beneficial system 1150 performance improvements over the conventional identical- 1151 throughput MIMO schemes. For example, SM uplink associ- 1152 ated with $N_{u}=4$ TAs/user and 4-QAM provides an SNR gain 1153 of about 4-5 dB over the conventional LS-MIMO uplink hav- 1154 ing $N_{u}=1 \mathrm{TA} /$ user and 16-QAM at BER $=10^{-3}$ for 16 users, 1155 $128 \mathrm{BS}$ antennas for a throughput of 4 bits/channel-use/user. 1156

The investigations of [105] were further extended in [78], 1157 where the generalized SM - GSM concept was considered 1158 for the sake of achieving increased-rate data transmission. 1159 Furthermore, the authors of [78] provided a closed-form ABEP 1160 upper bound expression based on the conventional union-bound 1161 method and developed a pair of extensions for the graph-based 1162 MP detector designed for SC-GSM signal detection operating 1163 in frequency flat-fading channels. It was shown in [78] that the 1164 proposed detectors exhibit a considerably reduced complexity, 1165 while providing a near-optimal BER performance.

1166

Moreover, the GSM-based system model of [78] was fur- 1167 ther developed for frequency-selective fading channels. In order 1168 to directly use the MP-based detector designed for the flat- 1169 fading case, it was combined with a CP-based SC technique 1170 for conceiving an equivalent system model. The numerical 1171 results demonstrated [78] that the GSM scheme associated with 1172 4 TAs/user, 4-QAM and 2 RF chains provides an SNR gain 1173 of about $12 \mathrm{~dB}$ over the conventional LS-MIMO having one 1174 TA/user and 64-QAM at BER $=10^{-3}$ for 16 users, $128 \mathrm{BS}$ anten- 1175 nas, a throughput of 4 bits/channel-use/user, and a CIR length 1176 of $L=3$. Moreover, it was found that [78] the proposed MP- 1177 based detectors are capable of improving the attainable BER 1178 performance, despite their reduced complexity, when compared 1179 to the classic MMSE detector. 
TABLE. VII

Major Contributions on SC-SM ANd Its LARGE-SCALE MiMO DEVElopments

\begin{tabular}{|c|c|c|c|c|c|c|c|}
\hline Literature & $\begin{array}{l}\text { Prefix } \\
\text { scheme }\end{array}$ & $\begin{array}{l}\text { Transmission } \\
\text { type }\end{array}$ & $\begin{array}{l}\text { Channel } \\
\text { coding }\end{array}$ & $\begin{array}{l}\text { Channel } \\
\text { model }\end{array}$ & $\begin{array}{l}\text { RF } \\
\text { required }\end{array}$ & $\begin{array}{l}\text { CSI } \\
\text { estimation }\end{array}$ & $\begin{array}{l}\text { Receiver } \\
\text { design }\end{array}$ \\
\hline $\begin{array}{l}\text { Narayanan } \\
\text { et al. }[100]\end{array}$ & N. A. & $\begin{array}{l}\text { MU downlink, } \\
\text { SM-MIMO }\end{array}$ & N. A. & Flat-fading & $\begin{array}{l}\text { User: } 1 \\
\text { BS: } U\end{array}$ & Perfect CSI & $\begin{array}{l}\text { Single-user } \\
\text { ML detector }\end{array}$ \\
\hline $\begin{array}{l}\text { Humadi } \\
\text { et al. }[101]\end{array}$ & N. A. & $\begin{array}{l}\text { MU downlink, } \\
\text { SM-MIMO }\end{array}$ & N. A. & $\begin{array}{l}\text { Flat-fading } \\
\text { Flat-fading }\end{array}$ & $\begin{array}{l}\text { User: } 1 \\
\text { BS: } N_{\text {tot }}\end{array}$ & Perfect CSI & ML detector \\
\hline $\begin{array}{l}\text { Narasimhan } \\
\text { et al. }[105]\end{array}$ & N. A. & $\begin{array}{l}\text { MU uplink, } \\
\text { SM-MIMO }\end{array}$ & N. A. & Flat-fading & $\begin{array}{l}\text { User: } 1 \\
\text { BS: } N_{\text {tot }}\end{array}$ & Perfect CSI & $\begin{array}{l}\text { MP and local } \\
\text { search detectors }\end{array}$ \\
\hline $\begin{array}{l}\text { Sugiura and } \\
\text { Hanzo [87] }\end{array}$ & $\mathrm{CP}$ & $\begin{array}{l}\text { Point-to-Point, } \\
\text { SM-MIMO }\end{array}$ & $\begin{array}{l}\text { Convolutional } \\
\text { code }\end{array}$ & $\begin{array}{l}\text { Frequency } \\
\text { selective } \\
\text { channel }\end{array}$ & Transmitter: 1 & Perfect CSI & $\begin{array}{l}\text { Soft MMSE } \\
\text { detector and } \\
\text { TEQ }\end{array}$ \\
\hline $\begin{array}{l}\text { Zheng } \\
\text { et al. [79] }\end{array}$ & N. A. & $\begin{array}{l}\text { Point-to-Point, } \\
\text { SM-MIMO }\end{array}$ & N. A. & $\begin{array}{l}\text { Frequency } \\
\text { selective } \\
\text { channel }\end{array}$ & Transmitter: 1 & Perfect CSI & ML detector \\
\hline $\begin{array}{l}\text { Xiao } \\
\text { et al. [88] }\end{array}$ & $\mathrm{ZP}$ & $\begin{array}{l}\text { Point-to-Point, } \\
\text { GSM-MIMO }\end{array}$ & N. A. & $\begin{array}{l}\text { Frequency } \\
\text { selective } \\
\text { channel }\end{array}$ & $\begin{array}{l}\text { Transmitter: } \\
1 \sim\left(N_{t}-1\right)\end{array}$ & Perfect CSI & LSS detector \\
\hline $\begin{array}{l}\text { Garcia } \\
\text { et al. }[107]\end{array}$ & N. A. & $\begin{array}{l}\text { MU uplink } \\
\text { SM-MIMO }\end{array}$ & N. A. & Flat Fading & $\begin{array}{l}\text { User: } 1 \\
\text { BS: } N_{\text {tot }}\end{array}$ & $\begin{array}{l}\text { Perfect CSI, } \\
\text { MMSE estimation }\end{array}$ & $\begin{array}{l}\text { Low complexity } \\
\text { CS detector }\end{array}$ \\
\hline $\begin{array}{l}\text { Wang } \\
\text { et al. }[113]\end{array}$ & $\mathrm{CP}$ & $\begin{array}{l}\text { MU uplink, } \\
\text { SM-MIMO }\end{array}$ & N. A. & $\begin{array}{l}\text { Frequency } \\
\text { selective } \\
\text { channel }\end{array}$ & $\begin{array}{l}\text { User: } 1 \\
\text { BS: } N_{\text {tot }}\end{array}$ & $\begin{array}{l}\text { Least square } \\
\text { estimation }\end{array}$ & $\begin{array}{l}\text { Factor graph } \\
\text { and MP-based } \\
\text { detectors }\end{array}$ \\
\hline
\end{tabular}

$U$ : the number of users.

$N_{\text {tot }}$ : the number of antennas at BS for the MU transmission.

$N_{\mathrm{u}}$ : the number of antennas at each user for the MU transmission.

$N_{t}$ : the number of antennas at transmitter for the point-to-point transmission.

In [112], the MP detector of [105] was further developed for employment in CP-aided SC-SM, where both the sparsity and the probability distribution of the transmit signals were exploited for reducing the MU detection complexity. As shown in [112] the most complex operation required for the proposed detector is constituted by parallelized matrixvector multiplications, which can be readily implemented in practice. In [112] the un-coded BER of the proposed MPbased detector was evaluated by applying the state evolution (SE) method, which is widely used to predict the performance of MP-like algorithms. Simulation results in [112] showed that the proposed MP algorithm is capable of achieving the near-ML performance. Moreover, an energy-efficient SC-SM scheme was proposed [112], which adjusts the transmit parameters for optimizing the energy consumption at a predefined target BER.
Based on the system model of [112], in [113] the authors 1197 investigated the MU detection issues in more practical scenar- 1198 ios, where the BS was equipped with low-resolution analog- 1199 to-digital convertors (ADCs), only impose a low circuit-power 1200 consumption on the LS-MIMO system. In [113], the classic 1201 least-square channel estimator was invoked and a novel low- 1202 complexity MP de-quantization detector was proposed, relying 1203 on the clustered factor graph method and the central limit the- 1204 orem. The simulation results of [113] have shown that the 1205 proposed detector outperforms the existing linear detectors and 1206 can efficiently operate under realistic LS-MIMO channel con- 1207 ditions, when the antennas are insufficiently far apart to avoid 1208 correlated fading.

In [107] a low complexity detector was designed for the 1210 uplink of LS-MIMO systems using SC-SM, which was based 1211 on a CS approach. It was shown that the signal structure of SM 1212 

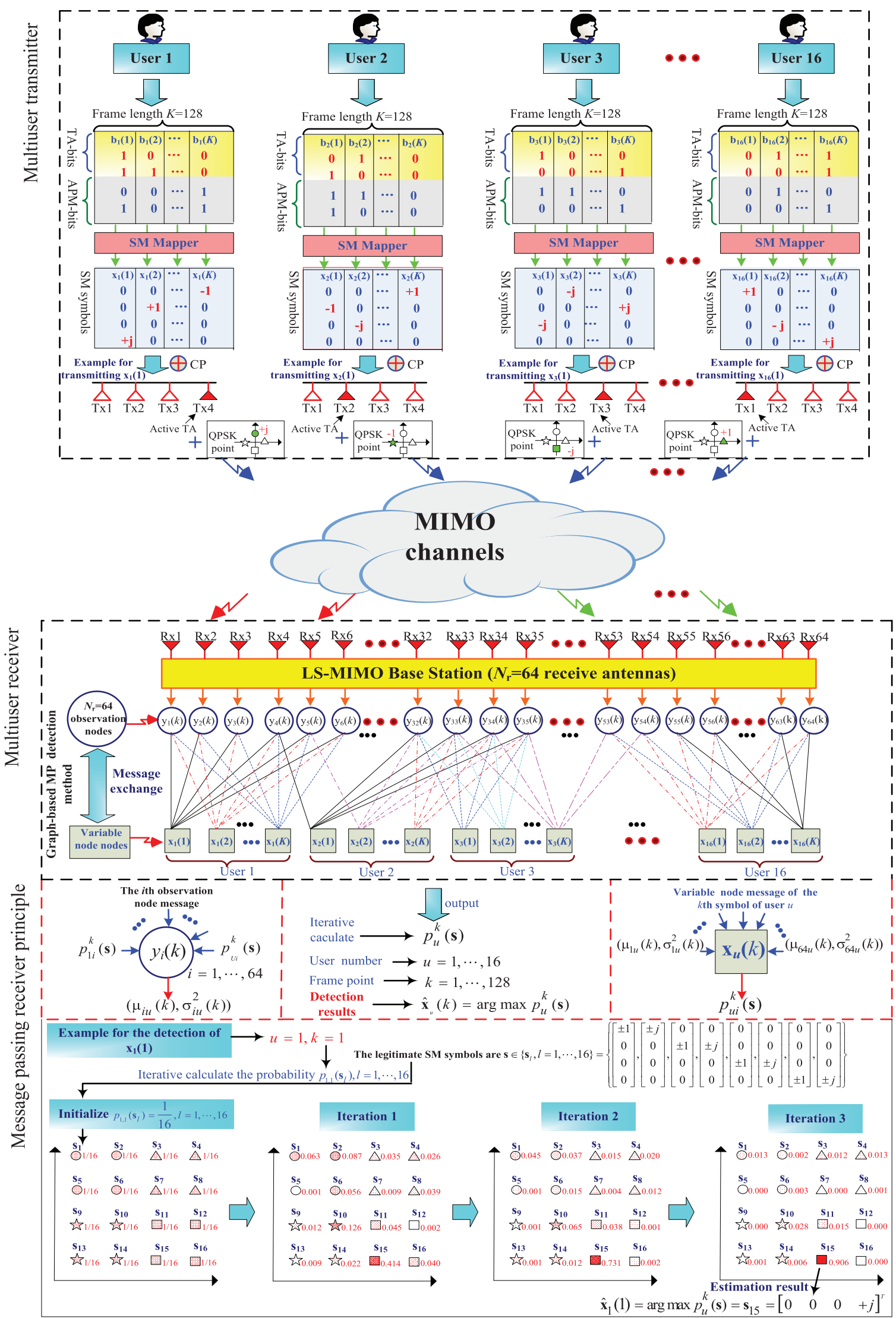

Fig. 13. An example of CP-aided SC-SM LS-MIMO system associated with $U=16, K=128, N_{t o t}=64, N_{u}=4$ and QPSK modulation, employing the MP-based detector. 
in the multiple access channel can be exploited for providing additional information and for improving the performance of the CS algorithms. The simple SC-SM detector was shown to offer a substantially improved energy-efficiency compared both to classical MIMO detectors and to conventional SM detection approaches.

\section{Uplink SC-SM LS-MIMO System Example and Performance Results}

In Fig. 13, we provide an example for an uplink SC-SM LSMIMO transmission scheme, which has $N_{\text {tot }}=64$ antennas at the BS and $U$ active users. Each user is equipped with $N_{u}=4$ TAs. For all users, we assume that the length of transmit frame is $K=128$ and QPSK modulation $\left(M_{\mathrm{SM}}=4\right)$ is employed for each TA. Based on the SC-SM framework in Fig. 2 and Table I, it is easy to show that the throughput of this scheme is $m_{\mathrm{SM}}$ $=\log _{2}\left(N_{u} M_{\mathrm{SM}}\right)=\log _{2}(4 \times 4)=4$ bits/channel-use/user, in which $\log _{2}\left(N_{u}\right)=2$ TA-bits are conveyed by TA indices and another $\log _{2}\left(M_{\mathrm{SM}}\right)=2$ APM-bits are conveyed by the APM symbols. Moreover, the following SM mapper is used: all possible pairs of TA-bit combinations $\left\{\left[\begin{array}{ll}0 & 0\end{array}\right],\left[\begin{array}{ll}0 & 1\end{array}\right],\left[\begin{array}{ll}1 & 0\end{array}\right],\left[\begin{array}{ll}1 & 1\end{array}\right]\right\}$ are mapped to the TA indices of $\{1,2,3,4\}$. Explicitly, TA 1 is activated when the input TA-bits are [0 0], TA 2 is activated when the input TA-bits are [0 1], TA 3 is activated when the input TA-bits are [1 0 ], and TA 4 is activated when the input TA-bits are [1 1]. Similarly, all possible APM-bit combinations $\left\{\left[\begin{array}{ll}0 & 0\end{array}\right],\left[\begin{array}{ll}0 & 1\end{array}\right],\left[\begin{array}{ll}1 & 1\end{array}\right],\left[\begin{array}{ll}1 & 0\end{array}\right]\right\}$ are one-to-one mapped to the classic QPSK constellation points $\{+1,+j,-1,-j\}$, i.e. $\left[\begin{array}{ll}0 & 0\end{array}\right]$ is mapped to the QPSK signal " +1 ” and [0 1] is mapped to QPSK signal "+j". Based on Algorithm 1 and [78], Fig. 13 shows the detailed operations in an SC-SM based MU transmitter as well as the corresponding MP algorithm based receiver for this 4 bits/channel-use/user system.

As shown in Fig. 13, 4 bits are transmitted in each channel use for each user, hence the information bit stream is divided into multiple 4-bit vectors, each of which is further divided into 2 TA-bits and 2 APM-bits. Then, based on the SM mapping rule described above, the two TA-bits are used to activate an unique TA for transmission, while the two APM-bits are mapped to a QPSK symbol. For example, in Fig. 13, the first bit vector $\mathbf{b}_{1}(1)=\left[\begin{array}{llll}1 & 1 & 0 & 1\end{array}\right]$ of user 1 is divided into the TA-bit vector [ 111$]$ and the APM-bit vector [0 1], which are mapped to TA 4 and the QPSK symbol $+j$, respectively. According to Eq. (1), the resultant SM symbol based on $\mathbf{b}_{1}(1)$ can be formulated as $\mathbf{x}_{1}(1)=$ $\left[\begin{array}{llll}0 & 0 & 0 & +j\end{array}\right]^{T} \in \mathbb{C}^{N_{t} \times 1}$. Fig. 13 shows the detailed transmitted frame generation process based on Algorithm 1 for each user and provides examples for TA activation and QPSK signal transmission for the first symbol $\mathbf{x}_{i}(1), i \in\{1, \cdots, 16\}$ of each user. An earlier example on the SC-SM signal generation process can also be found in Section II-B.

Recently, a graph-based MP algorithm has been proposed in [78]. To describe its operating principle, we consider the MP detection in the context of flat-fading channels, which can be readily extended to frequency-selective fading scenarios by formulating an equivalent channel model, as shown in [78]. Let $\overline{\mathbf{H}}(k) \in \mathbb{C}^{N_{\text {tot }} \times U N_{u}}$ denote the channel matrix in the $k$ th time slot, whose element $\bar{h}_{i,(u-1) N_{u}+j}(k)$ denotes the channel gain associated with the link spanning from the $j$ th TA of the $u$ th 1269 user to the $i$ th BS RA. The signal received at the BS of the $k$ th 1270 time slot is formulated by

$$
\begin{aligned}
y_{i}(k) & =\sum_{u=1}^{U} \overline{\mathbf{h}}_{i,[u]}(k) \mathbf{x}_{u}(k)+n_{i}(k) \\
& =\underbrace{\overline{\mathbf{h}}_{i,[u]}(k) \mathbf{x}_{u}(k)}_{\text {user } u}+\underbrace{\sum_{j=1, j \neq u}^{U} \overline{\mathbf{h}}_{i,[j]}(k) \mathbf{x}_{j}(k)}_{\text {MUI }}+\underbrace{n_{i}(k)}_{\text {noise }},
\end{aligned}
$$

where $\overline{\mathbf{h}}_{i,[u]}(k) \in \mathbb{C}^{1 \times N_{u}}$ is obtained from the $i$ th row of $\overline{\mathbf{H}}(k) 1272$ and of the $(u-1) N_{u}+1$ to the $u N_{u}$ columns of $\overline{\mathbf{H}}(k)$, with 1273 $n_{i}(k)$ being the noise term. As shown in Eq. (3), to detect the 1274 signal transmitted by the $u$ th user, the received signal $y_{i}(k)$ can 1275 be divided into three components: the component with useful 1276 information for user $u$, the MUI component from other users, 1277 and the noise component.

Based on Eq. (3), in Fig. 13 the MU SC-SM LS-MIMO 1279 system is represented as a factor graph. Observe in Fig. 131280 that the received signals $y_{i}(k), i=1, \cdots, 64$ can be viewed as 1281 the observation nodes, while the transmit symbols $\mathbf{x}_{u}(k), u=1282$ $1, \cdots, 16$ for the $k$ th time slot can be viewed as the vari- 1283 able nodes. The messages passed between variable nodes and 1284 observation nodes in the factor graph are approximated by a 1285 Gaussian distribution. To be specific, the summation of the 1286 MUI and noise term $N_{i u}(k)=\sum_{j=1, j \neq u}^{U} \overline{\mathbf{h}}_{i,[j]}(k) \mathbf{x}_{j}(k)+n_{i}(k) 1287$ of Eq. (3) is approximated as a Gaussian random variable with 1288 a mean of $\mu_{i u}(k)$ and a variance of $\sigma_{i u}^{2}(k)$.

In the graph-based MP-based detection, some specific 1290 messages are iteratively exchanged between the observation 1291 nodes $y_{i}(k), i=1, \cdots, 64$ and the variable nodes $\mathbf{x}_{u}(k), u=1292$ $1, \cdots, 16$, in order to achieve improved estimation result. In 1293 our example, the specific messages sending from an observa- 1294 tion node to a variable node are the scalar variables $\mu_{i u}(k) 1295$ and $\sigma_{i u}^{2}(k)$, which are the mean and variance of the sum of 1296 MUI and noise $N_{i u}(k)$, respectively. The message from vari- 1297 able nodes $\mathbf{x}_{u}(k)$ to observation nodes $y_{i}(k)$ is a vector about 1298 the $a$ posteriori probability $p_{u i}^{k}(\mathbf{s})$ of all possible transmitted 1299 SM symbols $\mathbf{s}$. Note that there are 16 legitimate SM symbols 1300 s for 4 bits/channel-use/user, which are given at the bottom of 1301 Fig. 13, the probability vector of which can be expressed as 1302 $\mathbf{p}_{u i}^{k}(\mathbf{s})=\left[p_{u i}^{k}\left(\mathbf{s}_{1}\right), p_{u i}^{k}\left(\mathbf{s}_{2}\right), \cdots, p_{u i}^{k}\left(\mathbf{s}_{16}\right)\right]$.

With the knowledge of $\mu_{i u}(k)$ and $\sigma_{i u}^{2}(k)$, the $a$ posteri- 1304 ori probability $p_{u i}^{k}(\mathbf{s})$ of all possible transmitted SM symbols 1305 $\mathbf{s}_{l}, l=1, \cdots, 16$ for the $u$ th user in the $k$ th time slot is given by 1306

$$
p_{u i}^{k}(\mathbf{s}) \propto \prod_{m=1, m \neq i}^{N_{\text {tot }}} \exp \left(\frac{-\left|y_{i}(k)-\mu_{m u}(k)-\overline{\mathbf{h}}_{m,[u]}(k) \mathbf{s}_{m}\right|^{2}}{2 \sigma_{m u}^{2}(k)}\right) .
$$

Note that the calculation of $p_{u i}^{k}(\mathbf{s})$ is independent of the 1307 observation node $y_{i}(k)$ itself, hence this message can be viewed 1308 as the extrinsic information, which is passed to the observation 1309 node $y_{i}(k)$ as the $a$ posteriori probability for estimation of the 1310 massages $\mu_{i u}(k)$ and $\sigma_{i u}^{2}(k)$ in the next iteration. The message 1311 
1312 exchanges between these factor nodes are iteratively carried out 1313

$$
p_{u}^{k}(\mathbf{s}) \propto \prod_{i=1}^{N_{t o t}} \exp \left(\frac{-\left|y_{i}(k)-\mu_{i u}(k)-\overline{\mathbf{h}}_{i,[u]}(k) \mathbf{s}\right|^{2}}{2 \sigma_{i u}^{2}(k)}\right) .
$$

1314 Compared to the extrinsic information $p_{u i}^{k}(\mathbf{s})$ in Eq. (4), the 1315 final decision metric $p_{u}^{k}(\mathbf{s})$ relied on the values of all observa1316 tion nodes. Finally, the detected SM signal of the $u$ th user in the $1317 k$ th time slot is formulated as:

$$
\hat{\mathbf{x}}_{u}(k)=\underset{\mathbf{s}}{\arg \max } p_{u}^{k}(\mathbf{s}) .
$$

Then, $\hat{\mathbf{x}}_{u}(k)$ is demodulated by the SM demapper to the original data bit sequence.

In Fig. 13, we give a simple example for the detection of $\mathbf{x}_{1}(1)=\left[\begin{array}{llll}0 & 0 & 0 & +j\end{array}\right]$ of user 1 by employing the abovementioned graph-based MP algorithm. According to Eq. (6), the detection of $\mathbf{x}_{1}(1)$ relies on the probabilities $p_{1}^{1}\left(\mathbf{s}_{l}\right), l=$ $1, \cdots, 16$, calculated by Eq. (5). In the initial stage of message exchange, all of these values are set to be $\frac{1}{16}$. Then, as the number of iterations increases, the MP algorithm begins to acquire these metrics $p_{1}^{1}\left(\mathbf{s}_{l}\right), l=1, \cdots, 16$ by updating the massages of $\mu_{i u}(k), \sigma_{i u}^{2}(k)$ and $p_{u i}^{k}(\mathbf{s})$. As shown in Fig. 13, after about 3 iterations, the value of $p_{1}^{1}\left(\mathbf{s}_{15}\right)$ becomes the highest in the set of $p_{1}^{1}\left(\mathbf{s}_{l}\right), l=1, \cdots, 16$. To be specific, it is equal to 0.414 in the first iteration, increases to 0.731 after the second iteration and reaches 0.906 after the third iteration. Based on the example of Fig. 13 and on Eq. (6), it is found that the SM symbol $\mathbf{s}_{15}$ is the final estimation result, which corresponds to the correct detection of $\mathbf{x}_{1}$ (1).

Based on a similar setup to that seen in Fig. 13 and [78], in Fig. 14 we evaluated the BER performance of a multiuser CP-aided SC-SM LS-MIMO system associated with $U=16$, $K=128, N_{r}=64, N_{t}=4$ and QPSK modulation for transmission over the EVA model, where the MP-based detector of [78] is employed. The number of iterations (denoted by iter) employed by the MP-based detector is 8. Furthermore, the conventional MMSE-based multiuser detector is also considered as a benchmark. As shown in Fig. 14, the MP algorithm has converged after 7 iterations, since the BER performance of iter $=$ 7 and iter $=8$ is almost identical. As expected, the MP-based detector is capable of efficiently detecting the SM symbols in large dimensions, as seen in Fig. 14, which outperformed the conventional MMSE detector by about $5 \mathrm{~dB}$ at a BER of $10^{-3}$ after 8 iterations. Moreover, as shown in [78], in addition to having this BER gain, the MP-based detector exhibits a considerably lower complexity than that of the MMSE-based detector, because it uses a low-complexity iterative message exchange process for avoiding the high-complexity channel inversion of the MMSE scheme. To be specific, the approximate complexity order of the MP-based detector is $\mathcal{O}\left(N_{\text {tot }} K N_{u} M_{\mathrm{SM}}\right)$, while the complexity order of the MMSE-based detector is $\mathcal{O}\left(N_{\text {tot }}^{2} K N_{u}\right)$. The MP-based detection exhibits considerable complexity reduction, since we have $N_{t o t}>>N_{u} M_{\mathrm{SM}}$ in LSMIMO systems.
Based on Table VII and our example, we can see that the 1361 above-mentioned SC-SM designs for LS-MIMO uplink trans- 1362 missions are more focused on the MU detector designs for the 1363 family of CP-aided SC-SM schemes. By contrast, as shown 1364 in Section II, the family of ZP-based SC-SM schemes may 1365 be preferred over its CP-based counterpart and further inves- 1366 tigations about the ZP-aided SC-SM designs are required. 1367 In Table VIII, we compare the throughput and the number 1368 of RF chains required for different MU LS-MIMO schemes, 1369 where the SC-GSM based MU LS-MIMO is capable of strik- 1370 ing a flexible throughput versus RF cost tradeoff. Moreover, 1371 in [78], a BER comparison between the MU SC-GSM LS- 1372 MIMO and MU SIMO LS-MIMO was presented to study the 1373 advantages of SC-SM schemes. It was shown that the MU 1374 SC-GSM LS-MIMO outperforms the conventional MU SIMO 1375 LS-MIMO by about $10 \mathrm{~dB}$ in terms of SNR at the throughput of 1376 6 bits/channel-use/user. Nevertheless, current research results 1377 are still preliminary and further investigations are required to 1378 identify the benefits of the family of MU SC-SM LS-MIMO 1379 schemes over other LS-MIMO schemes.

1380

Furthermore, as discussed in Sections I and III, SM can be 1381 realized by using a single RF front-end, hence it has a high 1382 power efficiency in realistic BS models. As shown in [97], in 1383 flat-fading channels SM has a considerable power consumption 1384 gain compared to multi-RF chain aided MIMO arrangements 1385 (e.g., STBC and VBLAST). However, in multipath channels, 1386 it is more challenging to evaluate the capacity of SC-SM for 1387 energy efficiency evaluation. Whether the benefits of energy 1388 efficiency observed in narrowband SM are still retained in 1389 broadband MU LS-MIMO scenarios requires further justifica- 1390 tion. More detailed discussions and potential solutions in the 1391 uplink and downlink SM-based LS-MIMO systems will be 1392 provided in the next Section.

\section{Further DESIGN IsSUes}

1394

According to the above-mentioned advantages and based on 1395 the initial results, SC-SM may be deemed to be an attractive 1396 low-complexity, low-cost design option for the emerging family 1397 of LS-MIMO systems, which is still in its infancy. To make the 1398 SC-SM based LS-MIMO systems a commercial reality, there 1399 are still numerous open issues that have to be studied. Some 1400 of these challenges and their potential solutions are shown in 1401 Fig. 15 and will be discussed below.

\section{A. High-Speed Antenna Switching}

The single-RF chain based design of SC-SM schemes 1404 requires an agile RF switch. The basic effects of this switch 1405 should be carefully resolved, namely the potential data loss 1406 inflicted by the shaping filter and the energy efficiency loss of 1407 the power amplifier caused by the isolated pulses transmitted 1408 in the time-domain. To combat these limitations while rely- 1409 ing on less RF chains than the number of TA elements, in 1410 [117] the authors quantified the above-mentioned performance 1411 penalty in terms of the bandwidth efficiency reduction and pro- 1412 posed a practical RF chain switching method, which is capable 1413 of operating in conjunction with a longer pulse shape than 1414 


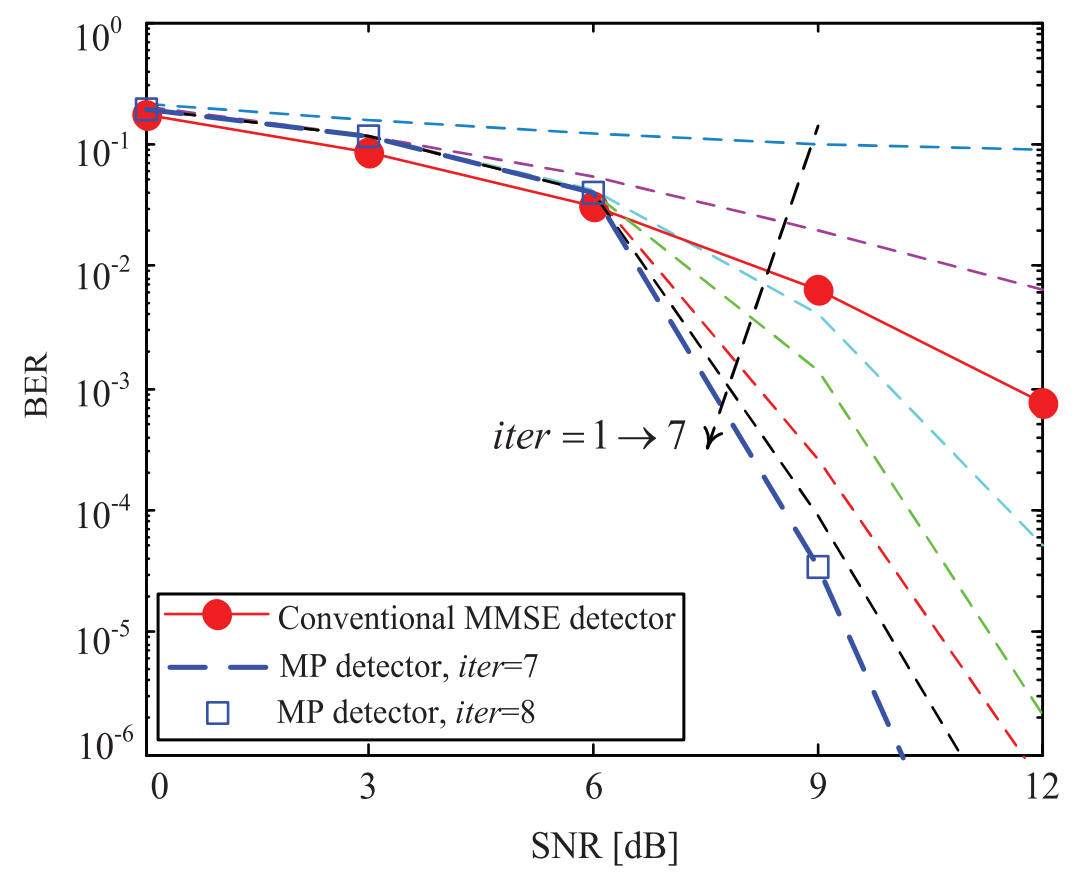

Fig. 14. BER performance of multiuser CP-aided SC-SM LS-MIMO system associated with $U=16, K=128, N_{\text {tot }}=64, N_{u}=4$ and QPSK modulation for transmission over the EVA model, where the MP-based detector is employed. The iteration number of MP-based detector is $i t e r=1-8$. Furthermore, the conventional MMSE-based multiuser detector is also consider as benchmarker

TABLE VIII

COMPARISON OF SC-SM, SC-GSM, SIMO, AND VBLAST BASED MU LS-MIMO UPLINK TRANSMISSION SYSTEMS

\begin{tabular}{|c|c|c|c|c|}
\hline Scheme & SC-SM LS-MIMO & SC-GSM LS-MIMO & SIMO LS-MIMO & VBLAST LS-MIMO \\
\hline Throughput & $T_{\mathrm{SM}}=$ & $T_{\mathrm{GSM}}=$ & $T_{\text {SIMO }}=$ & $T_{\mathrm{VBLAST}}=$ \\
\hline (bits/channel-use/user) & $\log _{2}\left(N_{u} M_{\mathrm{SM}}\right)$ & $\log _{2}\left(\begin{array}{l}N_{u} \\
N_{g}\end{array}\right)+N_{g} \log _{2}\left(M_{\mathrm{GSM}}\right)$ & $\log _{2}\left(M_{\mathrm{SIMO}}\right)$ & $N_{u} \log _{2}\left(M_{\text {VBLAST }}\right)$ \\
\hline RF chains/user & 1 & $N_{g}\left(N_{g}<N_{u}\right)$ & 1 & $N_{u}$ \\
\hline
\end{tabular}

$M_{\mathrm{GSM}}$ : the APM order in SC-GSM scheme.

$M_{\text {SIMO }}$ : the APM order in SIMO scheme.

$\lfloor\cdot\rfloor$ : the floor operator.

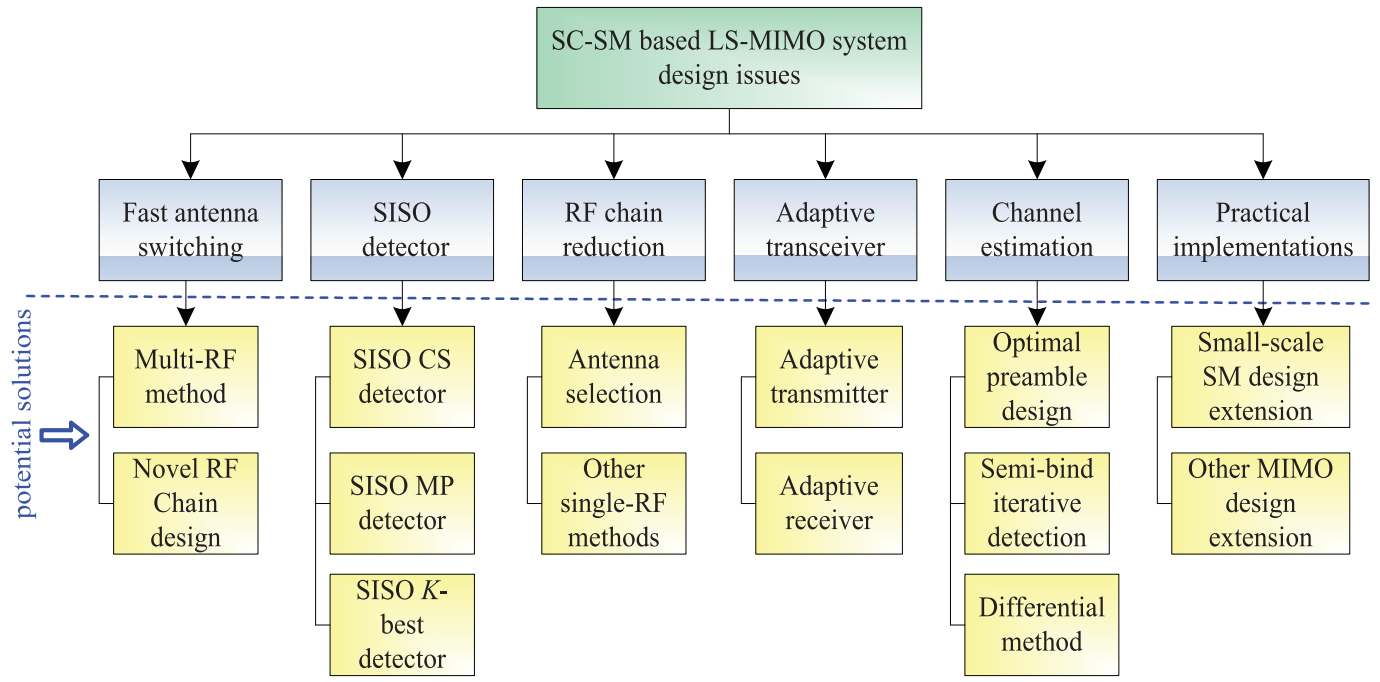

Fig. 15. The future design issues and the related potential solutions for the SC-SM based LS-MIMO.

that facilitated by a single-RF aided SM scheme. Moreover, in [118], a novel RF chain was designed for facilitating the smooth changing of the currents on the TAs, which constitutes an efficient alterative for pulse shaping technique. Another poten- 1418 tial design is the employment of low-complexity time-domain 1419 raised-cosine pulse shaping, which simply over-samples the 1420 
waveform by a sufficiently high factor and then smoothly transitions between the consecutive symbols. This can be attained, for instance, by reading the pre-stored intermediate values for all legitimate transitions from a look-up table [119]. However, to invoke these techniques in the context of SC-SM based LS-MIMO systems, more detailed investigations are needed.

\section{B. Soft-Input-Soft-Output Detector Design}

The signal dimension of SC-SM depends both on the frame length and on the number of antennas, which becomes high in the context of LS-MIMOs. There is a growing literature on how to design both linear and non-linear detectors for conventional LS-MIMO schemes, such as the lattice-based likelihood ascent search (LAS) [120] and the factor-graph based belief propagation algorithms [121]-[123]. However, they may not be directly applicable to the SC-SM based LS-MIMO schemes.

Here explicitly, most of the detectors designed for SC-SM in Section III may only be suitable for small-scale SC-SM systems, which commonly rely on 2-4 antennas at both ends of the wireless link. For large-scale SC-SM uplink transmission, the MP methods were proposed in [105], [112], [113], [124], but they are rely on hard decisions. However, in practice almost all wireless communication systems employ some form of FEC for enhancing the data reliability, which requires soft-input soft-output (SISO) detectors for achieving the highest possible coding gain.

Additionally, for the sake of reducing the computational complexity, most of the existing detectors were proposed for the family of CP-aided SC-SM schemes, where low-complexity single-tap equalization can be used, as shown in Table VII. However, this benefit is achieved under the constraint of $N_{t} \leq$ $N_{r}$, which erodes the performance of SM in asymmetric MIMO systems, routinely encountered in downlink transmission. In the future, the SISO detectors designed for high-diversity ZP-aided large-scale SC-SM have to be further investigated.

Since the generalized MP detectors of [105], [112], [113], [124], as well as the CS of [114], [115] and the sparse $K$ best detector of [116] provide significant detection complexity reductions by exploiting the inherent sparsity of the SC-SMtype signaling regime, these SISO versions may indeed be promising alternatives for SC-SM based LS-MIMO transmissions and they are worth more intensive study.

\section{Further RF Chain Reduction}

SM may be viewed as a special case of transmit antenna selection (AS). Unlike the AS techniques conceived for the conventional MIMO systems of [125], which rely on the channel quality often quantified in terms of the received signal strength, AS in SM is controlled by the incoming user data stream. SM enjoys the prominent benefit that the number of RF chains required is substantially reduced.

As shown in Section IV and Table VII, several attractive TPC schemes have been proposed for SC-SM based LS-MIMO downlink transmission systems [100], [101]. Although the ASlike SM scheme can reduce certain parts of RF chains, they still require a large number of $\mathrm{RF}$ chains at the $\mathrm{BS}$ for optimizing the precoder, which may jeopardize the most salient advan- 1475 tages of SC-SM. To further reduce the associated cost, the 1476 classic AS technique can be amalgamating the concept of SM 1477 for reducing the number of RF chains, without unduly erod- 1478 ing the attainable system performance. Especially, when a large 1479 number of antennas are used at the BS, the propagation chan- 1480 nel potentially provides much more spatial selectivity, than in 1481 small-scale scenarios. In this case, the beneficial system per- 1482 formance gain provided by the carefully designed AS schemes 1483 will also become more attractive.

1484

Note that in recent years various AS techniques have been 1485 proposed for the family of conventional SM schemes [126]- 1486 [132]. However, they have been designed for frequency flat- 1487 fading scenarios and hence may not be directly applicable to the 1488 more sophisticated SC-SM scheme. One of the key design chal- 1489 lenges of AS conceived for SC-SM based LS-MIMO systems 1490 is to construct a beneficial AS criterion, while relying both on a 1491 low-complexity and on a modest amount of feedback informa- 1492 tion. Moreover, a range of other RF chain reduction techniques 1493 may be combined with the SC-SM technique, in order to fur- 1494 ther reduce the cost of the hardware as exemplified by the 1495 single-RF based time-division multiplexing techniques and by 1496 the parasitic antenna method of [133].

\section{Practical Implementations}

1498

Experimental studies have also been conducted for evaluat- 1499 ing the SM-based transceiver in an indoor propagation scenario 1500 [56], [57], where the BER performance of small-scale MIMO 1501 channels (i.e. the $2 \times 2$ and the $4 \times 4$ MIMO configurations) 1502 operating both in LOS and non-LOS scenarios was inves- 1503 tigated. The measured results confirmed that the theoretical 1504 gains predicted by the analysis are substantiated by the Monte 1505 Carlo simulations [56]. Moreover, it was shown in [57] that 1506 the SM-based scheme performs better than the VBLAST and 1507 STBC schemes in the context of the measured channel models 1508 considered.

Note that these preliminary results were performed in a con- 1510 trolled laboratory environment in conjunction with a small 1511 number of antennas, where the effects of dispersive chan- 1512 nels and the impact of the associated hardware impairments 1513 have not been considered. On the other hand, for conventional 1514 LS-MIMO systems, the authors of [134] has provided some 1515 experimental results for SC modulation communicating over 1516 measured massive MIMO channels with 128 TAs. It was shown 1517 that the effects of ISI have to be carefully considered in con- 1518 ventional LS-MIMO systems, similar to the initial MU SC-SM 1519 based LS-MIMO design framework shown in Fig. 13. These 1520 observations and investigations may be extended to large-scale 1521 MU SC-SM schemes operating in realistic propagation sce- 1522 narios, so as to verify the related benefits of SC-SM in MU 1523 LS-MIMO systems.

\section{E. Adaptive Transceiver Design}

Adaptive transceivers play an important role in wireless com- 1526 munication systems, which are capable of dynamically adjust- 1527 ing the transceiver parameters in response to the time-variant 1528 
channel conditions [135], [136]. Hence they have been extensively studied in the conventional SM context for the sake of improving the achievable BER performance and of reducing the detection complexity [137]-[143], whilst relying on adaptive modulation [137], [138], on constellation optimization [54], [139]-[142], on power allocation [143], [144] as well as on phase rotation techniques [145]-[149]. However, it has not been considered, whether these adaptive techniques can be directly applied to the SC-SM based LS-MIMO systems. Even for the conventional LS-MIMO schemes, only limited initial work has been disseminated on the design of AS methods and on adaptive receivers [150], [151]. Near-capacity adaptive techniques, relying on low complexity and a low feedback requirement, are worth more intensive study.

\section{F. Channel Estimation and Low-Correlation Preamble} Sequence Design

A key design challenge in LS-MIMO systems (including SC-SM based LS-MIMO) is how to achieve optimal estimation of the CSI. A common approach to acquire CSI is to send properly designed preambles (or pilot signals) from the transmitter(s) and then estimate the channel impairments in the receiver by correlating the known preambles (locally generated) with the received signals. However, this process is always prone to estimation errors. For small-scale SM, the effects of these errors have been investigated in [41], [42], [152]-[155] and it was shown that SM is more robust to channel estimation errors than VBLAST. However, this advantage of SM has not been investigated in large-scale SC scenarios.

It is noted that optimal channel estimation in general requires the preamble sequences to have zero non-trivial auto- and cross-correlations [156]-[159]. However, the employment of conventional preamble sequences may jeopardize the advantages of SC-SM, since they have not been designed by taking into account the sparse structure of SC-SM symbols. To overcome this impediment, [112] and [113] developed the training sequences by using cyclic right-shifting method for SC-SM based on the MU channel estimation process, which was proposed for uplink LTE transmissions. However, this design may not be suitable for high-rate SC-SM schemes, owing to the associated power efficiency loss and the pilot overhead imposed. In all existing MIMO-oriented pilot sequences, the ternary-like pseudo-random sequences of [160] may be a promising candidate, including the family of SC-SM and SC-GSM schemes, because its spatial-domain sparsity can be flexibly adjusted. Nonetheless, additional work is necessary to make the associated design more practical.

In order to reduce the pilot-overhead, the semi-blind iterative detection technique of [161], the advanced training optimization techniques of [162] and the differentially encoded designs of [163]-[168] may be important future research directions.

\section{CONCLUSION}

In this paper, we reviewed a range of recent research achievements related to SC-SM, which constitutes a new low-complexity low-cost broadband MIMO transmission technique recently proposed. This novel scheme is capable of

adopting the low-complexity single-stream based detection, 1584 whilst relying on a single RF chain. Moreover, it can be 1585 designed for striking a flexible trade-off amongst the range of 1586 potentially conflicting system requirements, such as the effec- 1587 tive throughput, the diversity gain and the hardware cost, while 1588 facilitating communications over dispersive channels. The 1589 scheme reviewed here constitutes a promising candidate for 1590 LS-MIMO aided MU uplink and downlink design. However, 1591 for exploiting its full benefits numerous challenges have to be 1592 overcome.

\section{LIST OF ACRONYMS}

ABEP

ADCs

APM

APM-bits

AS

BER

BS

$\mathrm{CCDF}$

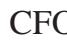

CIR

$\mathrm{CP}$

CS

CSI

DFT

DFE

EVA

FDE

FEC

FH-CDMA

\section{GSSK \\ GSM}

IBI

IFFT

KSP

LAS

LLRs

LOS

LSA

LS-MIMO

LSS

MAC

MAP

MF

MIMO

ML

MMSE

MP

MU

MUI

OFDM

PAPR

PIC

PIC-R-SIC
Average Bit Error Probability

Analog-to-Digital Convertors

Amplitude and Phase Modulation

Amplitude and Phase Modulation-bits

Antenna Selection

Bit Error Rate

Base Station

Complementary Cumulative Distribution

Function

Carrier Frequency Offset

Channel Impulse Response

Cyclic Prefix

Compressive Sensing

Channel State Information

Discrete Fourier Transform

Decision-Feedback Equalizer

Extended Vehicular A Model

Frequency-Domain Equalization

Forward Error Correction

Frequency Hopping Code Division Multiple Access

\section{Generalized Space Shift Keying}

Generalized Spatial Modulation

Inter-Block Interference

Inverse Fast Fourier Transform

Known Symbol Padding

Likelihood Ascent Search

Log-Likelihood Ratios

Line of Sight

Large-Scale Antenna

Large-Scale Multiple-Input Multiple-Output

Low-Complexity Single-Stream

Multiple Access Channel

Maximum A Posteriori

Matched Filter

Multiple-Input Multiple-Output

Maximum-Likelihood

Minimum Mean-Squared Error

Message Passing

Multiuser

Multiuser-Interference

Orthogonal Frequency-Division Multiplexing

Peak-to-Average Power Ratio

Parallel Interference Cancellation

PIC-based Receiver With SIC
1595

1596

1597

1598

1599

1600

1601

1602

1603

1604

1605

1606

1607

1608

1609

1610

1611

1612

1613

1614

1615

1616

1617

1618

1619

1620

1621

1622

1623

1624

1625

1626

1627

1628

1629

1630

1631

1632

1633

1634

1635

1636

1637

1638

1639 


$\begin{array}{lll}1640 & \text { QRD- } M & \text { QR Decomposition With } M \text {-algorithm } \\ 1641 & \text { RA } & \text { Receive Antenna } \\ 1642 & \text { RF } & \text { Radio Frequency } \\ 1643 & \text { RSM } & \text { Receiver-Side Spatial Modulation } \\ 1644 & \text { SC } & \text { Single-Carrier } \\ 1645 & \text { SC-FDE } & \text { Single-Carrier Systems Using } \\ 1646 & & \text { Frequency-Domain Equalization } \\ 1647 & \text { SC-FDMA } & \text { Single-Carrier Frequency Division Multiple } \\ 1648 & & \text { Access } \\ 1649 & \text { SC-SM } & \text { Single-Carrier-Based Spatial Modulation } \\ 1650 & \text { SD } & \text { Sphere Decoding } \\ 1651 & \text { SE } & \text { State Evolution } \\ 1652 & \text { SER } & \text { Symbol Error Rate } \\ 1653 & \text { SFSK } & \text { Space Frequency Shift Keying } \\ 1654 & \text { SIMO } & \text { Single-Input Multiple-Output } \\ 1655 & \text { SISO } & \text { Soft-Input Soft-Output } \\ 1656 & \text { SIC } & \text { Successive Interference Cancellation } \\ 1657 & \text { SM } & \text { Spatial Modulation } \\ 1658 & \text { SNR } & \text { Signal-to-Noise Ratio } \\ 1659 & \text { SSK } & \text { Space Shift Keying } \\ 1660 & \text { STdM } & \text { Space and Time-dispersion Modulation } \\ 1661 & \text { STBC } & \text { Space Time Block Code } \\ 1662 & \text { TA } & \text { Transmit Antenna } \\ 1663 & \text { TA-bits } & \text { Transmit Antenna-bits } \\ 1664 & \text { TDE } & \text { Time-Domain Equalizer } \\ 1665 & \text { TEQ } & \text { Turbo Equalizer } \\ 1666 & \text { TPC } & \text { Transmit Precoder } \\ 1667 & \text { VBLAST } & \text { Vertical Bell Labs Layered Space-Time } \\ 1668 & \text { ZF } & \text { Zero Forcing } \\ 1669 & \text { ZP } & \text { Zero Padding } \\ & & \end{array}$

1670

\section{REFERENCES}

[1] J. Mietzner, R. Schober, L. Lampe, W. H. Gerstacker, and P. A. Hoeher, "Multiple-antenna techniques for wireless communications-A comprehensive literature survey," IEEE Commun. Surv. Tuts., vol. 11, no. 2, pp. 87-105, Jun. 2009.

[2] S. Sugiura, S. Chen, and L. Hanzo, "A universal space-time architecture for multiple-antenna aided systems," IEEE Commun. Surv. Tuts., vol. 14, no. 2, pp. 401-420, Jan. 2012.

[3] L. Zheng and D. N. C. Tse, "Diversity and multiplexing: A fundamental tradeoff in multiple-antenna channels," IEEE Trans. Inf. Theory, vol. 49, no. 5, pp. 1073-1096, May 2003.

[4] B. Hassibi and B. M. Hochwald, "High-rate codes that are linear in space and time," IEEE Trans. Inf. Theory, vol. 48, no. 7, pp. 1804-1824, Jul. 2002.

[5] V. Tarokh, H. Jafarkhani, and A. R. Calderbank, "Space-time block codes from orthogonal designs," IEEE Trans. Inf. Theory, vol. 45, no. 5, pp. 1456-1467, Jul. 1999.

[6] A. Chockalingam and B. S. Rajan, Large MIMO Systems. Cambridge, U.K.: Cambridge Univ. Press, 2014.

[7] F. Rusek et al., "Scaling up MIMO: Opportunities and challenges with very large arrays," IEEE Signal Process. Mag., vol. 30, no. 1, pp. 40-60, Jan. 2013

[8] T. L. Marzetta, "Noncooperative cellular wireless with unlimited numbers of base station antennas," IEEE Trans. Wireless Commun., vol. 9 , no. 11 , pp. 3590-3600, Nov. 2010

[9] A. L. Swindlehurst, E. Ayanoglu, P. Heydari, and F. Capolino, "Millimeter-wave massive MIMO: The next wireless revolution?" IEEE Commun. Mag., vol. 52, no. 9, pp. 56-62, Sep. 2014.

[10] E. Larsson, O. Edfors, F. Tufvesson, and T. Marzetta, "Massive MIMO for next generation wireless systems," IEEE Commun. Mag., vol. 52, no. 2, pp. 186-195, Feb. 2014.

[11] J. Hoydis, S. ten Brink, and M. Debbah, "Massive MIMO in the UL/DL of cellular networks: How many antennas do we need?" IEEE J. Sel. Areas Commun., vol. 31, no. 2, pp. 160-171, Feb. 2013.
[12] K. Zheng, L. Zhao, J. Mei, B. Shao, W. Xiang, and L. Hanzo, "Survey of 1704 large-scale MIMO systems," IEEE Commun. Surv. Tuts., vol. 17, no. 3, 1705 pp. 1738-1760, Apr. 2015.

[13] M. Wu et al., "Large-scale MIMO detection for 3GPP LTE: Algorithms 1707 and FPGA implementations," IEEE J. Sel. Topics Signal Process., vol. 8, 1708 no. 5, pp. 916-929, Oct. 2014.

[14] D. Feng, C. Jiang, G. Lim, L. J. Cimini Jr., G. Feng, and G. Y. Li, “A 1710 survey of energy-efficient wireless communications," IEEE Commun. 1711 Surv. Tuts., vol. 15, no. 1, pp. 167-178, Feb. 2013. 1712

[15] G. Y. Li et al., "Energy-efficient wireless communications: Tutorial, sur- 1713 vey, and open issues," IEEE Wireless Commun. Mag., vol. 18, no. 6, 1714 pp. 28-35, Dec. 2011

[16] R. Y. Mesleh, H. Haas, S. Sinanovic, C. W. Ahn, and S. Yun, "Spatial 1716 modulation," IEEE Trans. Veh. Technol., vol. 57, no. 4, pp. 2228-2241, 1717 Jul. 2008.

[17] M. Di Renzo, H. Haas, and P. M. Grant, "Spatial modulation for 1719 multiple-antenna wireless systems: A survey," IEEE Commun. Mag., 1720 vol. 49, no. 12, pp. 182-191, Dec. 2011.

[18] M. Di Renzo, H. Haas, A. Ghrayeb, S. Sugiura, and L. Hanzo, 1722 "Spatial modulation for generalized MIMO: Challenges, opportunities 1723 and implementation," Proc. IEEE, vol. 102, no. 1, pp. 56-103, Jan. 1724 2014

[19] P. Yang, M. Di Renzo, Y. Xiao, S. Li, and L. Hanzo, "Design guidelines 1726 for spatial modulation," IEEE Commun. Surv. Tuts., vol. 17, no. 1, pp. 6- 1727 26, Mar. 2015

[20] D. J. Goodman, P. S. Henry, and V. K. Prabhu, "Frequency-hopped mul- 1729 tilevel FSK for mobile radio," Bell Syst. Tech. J., vol. 59, pp. 1257-1275, 1730 Sep. 1980.

1731

[21] T. Kawahara and T. Matsumoto, "Optimum rate reed Solomon codes 1732 for FFH/CDMA mobile radios," IEE Electron. Lett., vo1. 27, no. 21, 1733 pp. 2066-2067, Oct. 1991

[22] T. Kawahara and T. Matsumoto, "Optimal rate error protection for 1735 coded M-ary FSK FFH/CDMA mobile radio channels," in Proc. 1736 IEEE 42nd Veh. Technol. Conf. (VTC), Denver, CO, USA, 1992, 1737 pp. 396-399.

[23] R. Y. Mesleh, H. Haas, C. W. Ahn, and S. Yun, "Spatial modulation- 1739 A new low complexity spectral efficiency enhancing technique," in 1740 Proc. IEEE Int. Conf. Commun. Netw. China, Beijing, China, Oct. 2006, 1741 pp. 1-5.

1742

[24] R. Y. Mesleh, M. Di Renzo, H. Haas, and P. M. Grant, "Trellis coded 1743 spatial modulation," IEEE Trans. Wireless Commun., vol. 9, no. 7, 1744 pp. 2349-2361, Jul. 2010.

[25] R. Mesleh, H. Elgala, and H. Haas, "Optical spatial modulation," 1746 IEEE/OSA J. Opt. Commun. Netw., vol. 3, no. 3, pp. 234-244, Mar. 1747 2011.

[26] Y. Yang and B. Jiao, "Information-guided channel-hopping for high 1749 data rate wireless communication," IEEE Commun. Lett., vol. 12, no. 4, 1750 pp. 225-227, Apr. 2008.

1751

[27] S. Sugiura, S. Chen, and L. Hanzo, "Generalized space-time shift 1752 keying designed for flexible diversity-, multiplexing- and complexity- 1753 tradeoffs," IEEE Trans. Wireless Commun., vol. 10, no. 4, pp. 1144- 1754 1153, Apr. 2011.

[28] M. D. Renzo and H. Haas, "On transmit diversity for spatial modulation 1756 MIMO: Impact of spatial constellation diagram and shaping filters at the 1757 transmitter," IEEE Trans. Veh. Technol., vol. 62, no. 6, pp. 2507-2531, 1758 Jul. 2013.

[29] M. Di Renzo and H. Haas, "Bit error probability of space shift 1760 keying MIMO over multiple-access independent fading channels," 1761 IEEE Trans. Veh. Technol., vol. 60, no. 8, pp. 3694-3711, Oct. 1762 2011

[30] M. Di Renzo and H. Haas, "Bit error probability of SM-MIMO over 1764 generalized fading channels," IEEE Trans. Veh. Technol., vol. 61, no. 3, 1765 pp. 1124-1144, Mar. 2012

1766

[31] M. Di Renzo and H. Haas, "Space shift keying (SSK-) MIMO over 1767 correlated Rician fading channels: Performance analysis and a new 1768 method for transmit-diversity," IEEE Trans. Commun., vol. 59, no. 1, 1769 pp. 116-129, Jan. 2011.

[32] M. Di Renzo and H. Haas, "Space shift keying (SSK) modulation with 1771 partial channel state information: Optimal detector and performance 1772 analysis over fading channels," IEEE Trans. Commun., vol. 58, no. 11, 1773 pp. 3196-3210, Nov. 2010.

[33] E. Başar, Ü. Aygölü, E. Panayırcı, and H. V. Poor, "New trellis code 1775 design for spatial modulation," IEEE Trans. Wireless Commun., vol. 10, 1776 no. 8, pp. 2670-2680, Aug. 2011.

[34] E. Başar, Ü. Aygölü, E. Panayırcı, and H. V. Poor, "Super-orthogonal 1778 trellis-coded spatial modulation," IET Commun., vol. 6, no. 17, 1779 pp. 2922-2932, Nov. 2012. 
[35] R. Y. Chang, S. J. Lin, and W. H. Chung, "Energy efficient transmission over space shift keying modulated MIMO channels," IEEE Trans. Commun., vol. 60, no. 10, pp. 2950-2959, Oct. 2012.

[36] R. Y. Chang, S. J. Lin, and W. H. Chung, "New space shift keying modulation with Hamming code-aided constellation design," IEEE Wireless Commun. Lett., vol. 1, no. 1, pp. 2-5, Feb. 2012

[37] J. Jeganathan, A. Ghrayeb, L. Szczecinski, and A. Ceron, "Space shift keying modulation for MIMO channels," IEEE Trans. Wireless Commun., vol. 8, no. 7, pp. 3692-3703, Jul. 2009.

[38] M. Di Renzo and H. Haas, "A general framework for performance analysis of space shift keying (SSK) modulation for MISO correlated Nakagami-m fading channels," IEEE Trans. Commun., vol. 58, no. 9 . pp. 2590-2603, Sep. 2010.

[39] M. Di Renzo and H. Haas, "Bit error probability of space modulation over Nakagami-m fading: Asymptotic analysis," IEEE Commun. Lett., vol. 15 , no. 10, pp. 1026-1028, Oct. 2011.

[40] M. T. Le, V. D. Ngo, H. A. Mai, X. N. Tran, and M. Di Renzo, "Spatially modulated orthogonal space-time block codes with non-vanishing determinants," IEEE Trans. Commun., vol. 62, no. 1, pp. 85-99, Jan. 2014.

[41] F. S. Al-Qahtani, S. Ikki, M. Di Renzo, and H. Alnuweiri, "Performance analysis of space shift keying modulation with imperfect estimation in the presence of co-channel interference," IEEE Commun. Lett., vol. 18, no. 9, pp. 1587-1590, Sep. 2014

[42] M. Di Renzo, D. De Leonardis, F. Graziosi, and H. Haas, "Space shift keying (SSK-) MIMO with practical channel estimates," IEEE Trans. Commun., vol. 60, no. 4, pp. 998-1012, Apr. 2012.

[43] M. Di Renzo and H. Haas, "Improving the performance of space shift keying (SSK) modulation via opportunistic power allocation," IEEE Commun. Lett., vol. 14, no. 6, pp. 500-502, Jun. 2010.

[44] F. S. Al-Qahtani, Y. Huang, M. Di Renzo, S. Ikki, and H. Alnuweiri, "Space shift keying MIMO system under spectrum sharing environments in Rayleigh fading," IEEE Commun. Lett., vol. 18, no. 9, pp. 1503-1506, Sep. 2014.

[45] N. Serafimovski, S. Sinanović, M. Di Renzo, and H. Haas, "Multiple access spatial modulation," EURASIP J. Wireless Commun. Netw. vol. 2012, no. 299, pp. 1-20, 2012, doi: 10.1186/1687-1499-2012-299.

[46] A. Stavridis, S. Sinanovic, M. Di Renzo, H. Haas, and P. M. Grant, "An energy saving base station employing spatial modulation," in Proc. IEEE Int. Workshop Comput. Aided Model. Anal. Design Commun. Links Netw., Barcelona, Spain, Sep. 2012, pp. 231-235.

[47] Y. Yang and S. Aissa, "Information-guided transmission in decode-and forward relaying systems: Spatial exploitation and throughput enhancement," IEEE Trans. Wireless Commun., vol. 10, no. 7, pp. 2341-2351, Jul. 2011.

[48] R. Mesleh, S. S. Ikki, E. M. Aggoune, and A. Mansour, "Performance analysis of space shift keying (SSK) modulation with multiple cooperative relays," EURASIP J. Adv. Signal Process., vol. 2012, pp. 1-10, Sep. 2012.

[49] R. Mesleh, S. S. Ikki, and M. Alwakeel, "Performance analysis of space shift keying with amplify and forward relaying," IEEE Commun. Lett., vol. 15 , no. 12 , pp. $1350-1352$, Dec. 2011

[50] N. Serafimovski, S. Sinanović, M. Di Renzo, and H. Haas, "Dual-hop spatial modulation (Dh-SM)," in Proc. Veh. Technol. Conf. (VTC'11Spring), Budapest, Hungary, May 15-18, 2011, pp. 1-5.

[51] K. Unnikrishnan and B. Rajan, "Space-time coded spatial modulated physical layer network coding for two-way relaying," IEEE Trans. Wireless Commun., vol. 14, no. 1, pp. 331-342, Jan. 2015

[52] P. Yang et al., "Detect-and-forward relaying aided cooperative spatial modulation for wireless networks," IEEE Trans. Commun., vol. 61, no. 11 , pp. 4500-4511, Nov. 2013.

[53] A. Stavridis et al., "A virtual MIMO dual-hop architecture based on hybrid spatial modulation," IEEE Trans. Commun., vol. 62, no. 9, pp. 3161-3179, Sep. 2014.

[54] K. Ntontin, M. Di Renzo, A. I. Perez-Neira, and C. Verikoukis, "Adaptive generalized space shift keying," EURASIP J. Wireless Commun. Netw., vol. 2013, no. 43, pp. 1-15, Feb. 2013

[55] A. Younis et al., "Performance of spatial modulation using measured real-world channels," in Proc. IEEE Veh. Technol. Conf., Las Vegas, NV, USA, 2013, pp. 1-5.

[56] N. Serafimovski et al., "Practical implementation of spatial modulation," IEEE Trans. Veh. Technol., vol. 62, no. 9, pp. 4511-4523, Nov. 2013.

[57] J. Zhang, Y. Wang, L. Ding, and N. Zhang, "Bit error probability of spatial modulation over measured indoor channels," IEEE Trans. Wireless Commun., vol. 13, no. 3, pp. 1380-1387, Mar. 2014.

[58] A. Goldsmith, Wireless Communications. Cambridge, U.K.: Cambridge Univ. Press, 2005.
[59] G. L. Stuber, J. Barry, S. McLaughlin, Y. G. Li, M. A. Ingram, and 1859 T. Pratt, "Broadband MIMO-OFDM wireless communications," Proc. 1860 IEEE, vol. 92, no. 2, pp. 271-294, Feb. 2004.

1861

[60] M. I. Kadir, S. Sugiura, S. Cheng, and L. Hanzo, "Unified MIMO- 1862 multicarrier designs: A space-time shift keying approach," IEEE 1863 Commun. Surv. Tuts., vol. 17, no. 2, pp. 550-579, Sep. 2015.

[61] B. Muquet, Z. Wang, G. Giannakis, M. de Courville, and P. Duhamel, 1865 "Cyclic prefixing or zero padding for wireless multicarrier transmis- 1866 sions?" IEEE Trans. Commun., vol. 50, no. 12, pp. 2136-2148, Dec. 1867 2002.

1868

[62] N. Benvenuto, R. Dinis, D. Falconer, and S. Tomasin, "Single car- 1869 rier modulation with nonlinear frequency domain equalization: An idea 1870 whose time has come-Again," Proc. IEEE, vol. 98, no. 1, pp. 69-96, 1871 Jan. 2010.

[63] A. Pitarokoilis, S. K. Mohammed, and E. G. Larsson, "On the opti- 1873 mality of single-carrier transmission in large-scale antenna systems," 1874 IEEE Wireless Commun. Lett., vol. 1, no. 4, pp. 276-279, Apr. 1875 2012.

[64] L. Lu, G. Y. Li, A. L. Swindlehurst, A. Ashikhmin, and R. Zhang, 1877 "An overview of massive MIMO: Benefits and challenges," IEEE 1878 J. Sel. Topics Signal Process., vol. 8, no. 5, pp. 742-758, Oct. 1879 2014.

[65] Y. Sheng, Z. Tan, and G. Y. Li, "Single-carrier modulation with 1881 ML equalization for large-scale antenna systems over Rician fading 1882 channels," in Proc. IEEE Int. Conf. Acoust. Speech Signal Process. 1883 (ICASSP), Florence, Italy, 2014, pp. 5759-5763.

[66] B. Zhou, Y. Xiao, P. Yang, J. Wang, and S. Li, "Spatial modulation 1885 for single carrier wireless transmission systems," in Proc. 6th Int. ICST 1886 Conf. Commun. Netw. China, Harbin, China, Aug. 2011, pp. 11-15. 1887

[67] N. Serafimovski, M. Di Renzo, S. Sinanović, R. Y. Mesleh, and H. Haas, 1888 "Fractional bit encoded spatial modulation (FBE-SM)," IEEE Commun. 1889 Lett., vol. 14, no. 5, pp. 429-431, May 2010.

1890

[68] Y. Yang and S. Aissa, "Bit-padding information guided channel hop- 1891 ping," IEEE Commun. Lett., vol. 15, no. 2, pp. 163-165, Feb. 2011. 1892

[69] A. G. Orozco-Lugo, M. M. Lara, and D. C. McLernon, "Channel esti- 1893 mation using implicit training," IEEE Trans. Signal Process., vol. 52, 1894 no. 1, pp. 240-254, Jan. 2004

1895

[70] P. Som and A. Chockalingam, "Spatial modulation and space shift 1896 keying in single carrier communication," in Proc. IEEE 23rd Int. 1897 Symp. Pres. Indoor Mobile Radio Commun. (PIMRC), Sydney, NSW, 1898 Australia, Sep. 2012, pp. 1961-1967.

1899

[71] R. Rajashekar, K. V. S. Hari, and L. Hanzo, "Spatial modulation aided 1900 zero-padded single carrier transmission for dispersive channels," IEEE 1901 Trans. Commun., vol. 61, no. 6, pp. 2318-2329, Jun. 2013.

[72] S. M. Alamouti, "A simple transmit diversity technique for wire- 1903 less communications," IEEE J. Sel. Areas Commun., vol. 16, no. 8, 1904 pp. 1451-1458, Oct. 1998.

[73] J. Fu, C. Hou, W. Xiang, L. Yan, and Y. Hou, "Generalised spatial 1906 modulation with multiple active transmit antennas," in Proc. IEEE 1907 GLOBECOM Workshops, Dec. 2010, pp. 839-844.

1908

[74] T. Datta and A. Chockalingam, "On generalized spatial modulation," 1909 in Proc. IEEE Wireless Commun. Netw. Conf. (WCNC'13), Apr. 2013, 1910 pp. 2716-2721.

[75] J. Wang, S. Jia, and J. Song, "Generalised spatial modulation system 1912 with multiple active transmit antennas and low complexity detection 1913 scheme," IEEE Trans. Wireless Commun., vol. 11, no. 4, pp. 1605-1615, 1914 Apr. 2012.

1915

[76] J. Jeganathan, A. Ghrayeb, and L. Szczecinski, "Generalized space 1916 shift keying modulation for MIMO channels," in Proc. IEEE Int. 1917 Symp. Pers. Indoor Mobile Radio Commun., Cannes, France, Sep. 2008, 1918 pp. $1-5$.

[77] S. Sugiura, S. Chen, and L. Hanzo, "Coherent and differential space time 1920 shift keying: A dispersion matrix approach," IEEE Trans. Commun., 1921 vol. 58, no. 11, pp. 3219-3230, Nov. 2010.

[78] T. L. Narasimhan, P. Raviteja, and A. Chockalingam, "Generalized spa- 1923 tial modulation in large-scale multiuser MIMO systems," IEEE Trans. 1924 Wireless Commun., vol. 24, no. 7, pp. 3764-3779, Jul. 2015.

[79] J. Zheng and Y. Sun, "Energy-efficient spatial modulation over MIMO 1926 frequency selective fading channels," IEEE Trans. Veh. Technol., vol. 64, 1927 no. 5, pp. 2204-2209, May 2015

[80] H. A. Ngo, C. Xu, S. Sugiura, and L. Hanzo, "Space-time-frequency 1929 shift keying for dispersive channels," IEEE Signal Process. Lett., vol. 18, 1930 no. 3, pp. 177-180, Mar. 2011.

[81] A. Younis, N. Serafimovski, R. Mesleh, and H. Haas, "Generalised spa- 1932 tial modulation," in Proc. Signals Syst. Comput., Pacific Grove, CA, 1933 USA, Nov. 2010, pp. 1498-1502.

[82] J. G. Proakis, Digital Communications, 4th ed. New York, NY, USA: 1935 McGraw-Hill, 2004 
[83] R. W. Heath Jr. and A. J. Paulraj, "Linear dispersion codes for MIMO systems based on frame theory," IEEE Trans. Signal Process., vol. 50, no. 10, pp. 2429-2441, Oct. 2002.

[84] J. Jeganathan, A. Ghrayeb, and L. Szczecinski, "Spatial modulation: Optimal detection and performance analysis," IEEE Commun. Lett., vol. 12 , no. 8, pp. 545-547, Aug. 2008.

[85] S. Sugiura, C. Xu, S. X. Ng, and L. Hanzo, "Reduced-complexity coherent versus non-coherent QAM-aided space-time shift keying," IEEE Trans. Commun., vol. 59, no. 11, pp. 3090-3101, Nov. 2011.

[86] S. Sugiura, S. Chen, and L. Hanzo, "MIMO-aided near-capacity turbo transceivers: Taxonomy and performance versus complexity," IEEE Commun. Surv. Tuts., vol. 14, no. 2, pp. 421-442, May 2012.

[87] S. Sugiura and L. Hanzo, "Single-RF spatial modulation requires single-carrier transmission: Frequency-domain turbo equalization for dispersive channels," IEEE Trans. Veh. Technol., vol. 64, no. 10, pp. $4870-4875$, Oct. 2015.

[88] L. Xiao, D. Lilin, Y. Zhang, Y. Xiao, P. Yang, and S. Li, "A lowcomplexity detection scheme for generalized spatial modulation aided single carrier systems," IEEE Commun. Lett., vol. 19, no. 6, pp. 10691072, Jun. 2015.

[89] J. Zhang, L. Yang, and L. Hanzo, "Advances in cooperative single-carrier FDMA communications: Beyond LTE-Advanced," IEEE Commun. Surv. Tuts., vol. 17, no. 2, pp. 730-756, May 2015

[90] F. Pancaldi, G. Vitetta, R. Kalbasi, N. Al-Dhahir, M. Uysal, and H. Mheidat, "Single-carrier frequency domain equalization," IEEE Signal Process. Mag., vol. 25, no. 5, pp. 37-56, Sep. 2008.

[91] A. H. Mehana and A. Nosratinia, "Diversity of MMSE MIMO receivers," IEEE Trans. Inf. Theory, vol. 51, no. 11, pp. 6788-6805, Nov. 2012.

[92] O. D. Damen, H. El Gamel, and G. Caire, "On maximum-likelihood detection and the search for the closest lattice point," IEEE Trans. Inf. Theory, vol. 47, no. 10, pp. 2389-2402, Oct. 2003.

[93] A. Younis, S. Sinanovic, M. Di Renzo, R. Y. Mesleh, and H. Haas, "Generalised sphere decoding for spatial modulation," IEEE Trans. Commun., vol. 61, no. 7, pp. 2805-2815, Jul. 2013.

[94] L. Hanzo, T. H. Liew, B. L. Yeap, R. Y. S. Tee, and S. X. Ng, Turbo Coding, Turbo Equalisation, and Space-Time Coding: EXIT-ChartAided Near-Capacity Designs for Wireless Channels, 2nd ed. Hoboken, NJ, USA: Wiley, 2011

[95] E. Candes, J. Romberg, and T. Tao, "Robust uncertainty principles: Exact signal reconstruction from highly incomplete frequency information," IEEE Trans. Inf. Theory, vol. 52, no. 2, pp. 489-509, Feb. 2006

[96] D. Donoho, "Compressed sensing," IEEE Trans. Inf. Theory, vol. 52, no. 4, pp. 1289-1306, Apr. 2006.

[97] A. Stavridis, S. Sinanovic, M. Di Renzo, and H. Haas, "Energy evaluation of spatial modulation at a multi-antenna base station," in Proc. IEEE Veh. Technol. Conf., Barcelona, Spain, Sep. 2013, pp. 1-5.

[98] E. Başar, Ü. Aygölü, E. Panayırc1, and H. V. Poor, "Space-time block coded spatial modulation," IEEE Trans. Commun., vol. 59, no. 3, pp. 823-832, Mar. 2011.

[99] 3GPP TR36.814, "Evolved universal terrestrial radio access (E-UTRA): Further advancements for E-UTRA physical layer aspects, v. 9.0.0, Mar. 2010.

[100] S. Narayanan, M. J. Chaudhry, A. Stavridis, M. Di Renzo, F. Graziosi, and H. Haas, "Multi-user spatial modulation MIMO," in Proc. IEEE Wireless Commun. Netw. Conf. (WCNC'14), Istanbul, Turkey, Apr. 2014, pp. 671-676.

[101] K. M. Humadi, A. I. Sulyman, and A. Alsanie, "Spatial modulation concept for massive multiuser MIMO systems," Int. J. Antenna Propag., vol. 2014, pp. 1-9, 2014

[102] R. Zhang, L. Yang, and L. Hanzo, "Generalised pre-coding aided spatial modulation," IEEE Trans. Wireless Commun., vol. 12, no. 11, pp. 5434 5443, Nov. 2013

[103] R. Zhang, L.-L. Yang, and L. Hanzo, "Error probability and capacity analysis of generalized pre-coding aided spatial modulation," IEEE Trans. Wireless Commun., vol. 14, no. 1, pp. 364-375, Jan. 2015.

[104] A. Stavridis, D. Basnayaka, S. Sinanovic, M. Di Renzo, and H. Haas, "A virtual MIMO dual-hop architecture based on hybrid spatial modulation," IEEE Trans. Commun., vol. 62, no. 9, pp. 3161-3179, Sep. 2014.

[105] T. L. Narasimhan, P. Raviteja, and A. Chockalingam, "Large-scale multiuser SM-MIMO versus massive MIMO," in Proc. Inf. Theory Appl. Workshop (ITA), Feb. 2014, pp. 56-62.

[106] J. Zheng, "Low-complexity detector for spatial modulation multiple access channels with a large number of receive antennas," IEEE Commun. Lett., vol. 18, no. 11, pp. 2055-2058, Nov. 2014.
[107] A. Garcia Rodriguez and C. Masouros, "Low-complexity compressive 2014 sensing detection for spatial modulation in large-scale multiple access 2015 channels," IEEE Trans. Commun., vol. 63, no. 7, pp. 2565-2579, Jul. 2016 2015.

[108] B. J. Frey, Graphical Models for Machine Learning and Digital 2018 Communication. Cambridge, MA, USA: MIT Press, 1998.

[109] R. J. McEliece, D. J. C. MacKay, and J.-F. Cheng, "Turbo decoding as 2020 an instance of Pearls "belief propagation" algorithm," IEEE J. Sel. Areas 2021 Commun., vol. 16, no. 2, pp. 140-152, Feb. 1998.

[110] T. L. Narasimhan and A. Chockalingam, "Channel hardening-exploiting 2023 message passing (CHEMP) receiver in large-scale MIMO systems," 2024 IEEE J. Sel. Topics Signal Process., vol. 8, no. 5, pp. 847-860, Oct. 2025 2014.

[111] B. J. Frey and D. Dueck, "Clustering by passing messages between data 2027 points," Science, vol. 315, pp. 972-976, Feb. 16, 2007.

2028

[112] S. Wang, Y. Li, M. Zhao, and J. Wang, "Energy efficient and low- 2029 complexity uplink transceiver for massive spatial modulation MIMO," 2030 IEEE Trans. Veh. Technol., vol. 64, no. 10, pp. 4617-4632, Oct. 2015. 2031

[113] S. Wang, Y. Li, and J. Wang, "Multiuser detection in massive spatial 2032 modulation MIMO with low-resolution ADCs," IEEE Trans. Wireless 2033 Commun., vol. 14, no. 4, pp. 2156-2168, Apr. 2015.

2034

[114] C. M. Yu et al., "Compressed sensing detector design for space shift 2035 keying in MIMO systems," IEEE Commun. Lett., vol. 16, no. 10, 2036 pp. 1556-1559, Oct. 2012.

2037

[115] W. Liu, N. Wang, M. Jin, and H. Xu, "Denoising detection for the gener- 2038 alized spatial modulation system using sparse property," IEEE Commun. 2039 Lett., vol. 18, no. 1, pp. 22-25, Jan. 2014.

[116] X. Peng, W. Wu, J. Sun, and Y. Liu, "Sparse K-best detector for gen- 2041 eralised space shift keying in large-scale multiple-input-multiple-output 2042 systems," IET Commun., vol. 9, no. 6, pp. 771-778, Apr. 2015.

[117] K. Ishibashi and S. Sugiura, "Effects of antenna switching on band- 2044 limited spatial modulation," IEEE Wireless Commun. Lett., vol. 3, no. 4, 2045 pp. 345-348, Apr. 2014.

2046

[118] M. S. Sedaghat, R. R. Mueller, and G. Fischer, "A novel single-RF 2047 transmitter for massive MIMO," in Proc. 18th Int. ITG Workshop Smart 2048 Antennas (WSA), Erlangen, Germany, Mar. 2014, pp. 1-8. 2049

[119] L. Hanzo, S. X. Ng, T. Keller, and W. Webb, Quadrature Amplitude 2050 Modulation: From Basics to Adaptive Trellis-Coded, Turbo-Equalised 2051 and Space-Time Coded OFDM, CDMA and MC-CDMA Systems. 2052 Hoboken, NJ, USA: Wiley/IEEE Press, 2004.

[120] K. V. Vardhan, S. K. Mohammed, A. Chockalingam, and B. S. Rajan, 2054 "A low-complexity detector for large MIMO systems and multicarrier 2055 CDMA systems," IEEE J. Sel. Areas Commun., vol. 26, no. 3, pp. 473- 2056 485, Apr. 2008.

[121] S. K. Mohammed, A. Zaki, A. Chockalingam, and B. S. Rajan, "High 2058 rate space time coded large-MIMO systems: Low-complexity detection 2059 and channel estimation," IEEE J. Sel. Topics Signal Process., vol. 3, 2060 no. 6, pp. 958-974, Dec. 2009.

2061

[122] N. Srinidhi, T. Datta, A. Chockalingam, and B. S. Rajan, "Layered tabu 2062 search algorithm for large-MIMO detection and a lower bound on ML 2063 performance," IEEE Trans. Commun., vol. 59, no. 11, pp. 2955-2963, 2064 Nov. 2011

2065

[123] T. Datta, N. A. Kumar, A. Chockalingam, and B. S. Rajan, "A novel 2066 Monte Carlo sampling based receiver for large-scale uplink multiuser 2067 MIMO systems," IEEE Trans. Veh. Technol., vol. 62, no. 7, pp. 3019- 2068 3038, Sep. 2013.

[124] P. Raviteja, T. L. Narasimhan, and A. Chockalingam, "Detection in large 2070 scale multiuser SM-MIMO systems: Algorithms and performance," in 2071 Proc. IEEE Veh. Technol. Conf. (VTC'14-Spring), May 2014, pp. 1-5. 2072

[125] S. Sanayei and A. Nosratinia, "Antenna selection in MIMO systems," 2073 IEEE Commun. Mag., vol. 42, no. 10, pp. 68-73, Oct. 2004.

[126] P. Zhang, S. Chen, C. Dong, L. Li, and L. Hanzo, "Two-tier channel esti- 2075 mation aided near-capacity MIMO transceivers relying on norm-based 2076 joint transmit and receive antenna selection," IEEE Trans. Wireless 2077 Commun., vol. 14, no. 1, pp. 122-137, Jan. 2015.

[127] Z. Zhou, N. Ge, and X. Lin, "Reduced-complexity antenna selection 2079 schemes in spatial modulation," IEEE Commun. Lett., vol. 18, no. 1, 2080 pp. 14-17, Jun. 2014.

[128] R. Rajashekar, K. V. S. Hari, and L. Hanzo, "Antenna selection in spatial 2082 modulation systems," IEEE Commun. Lett., vol. 17, no. 3, pp. 521-524, 2083 Mar. 2013.

[129] K. Ntontin, M. Di Renzo, A. Perez-Neira, and C. Verikoukis, "A low- 2085 complexity method for antenna selection in spatial modulation systems," 2086 IEEE Commun. Lett., vol. 17, no. 12, pp. 2312-2315, Aug. 2013. 2087

[130] N. Wang, W. Liu, H. Men, and M. Jin, and H. Xu. "Further complexity 2088 reduction using rotational symmetry for EDAS in spatial modulation," 2089 IEEE Commun. Lett., vol. 18, no. 10, pp. 1835-1838, Oct. 2014. 
[131] J. Zheng, "Fast receive antenna subset selection for pre-coding aided spatial modulation," IEEE Wireless Commun. Lett., vol. 4, no. 3, pp. 317-320, Jun. 2015.

[132] J. Zheng and J. Chen, "Further complexity reduction for antenna selection in spatial modulation systems," IEEE Commun. Lett., vol. 19, no. 6, pp. 937-940, Jun. 2015.

[133] A. Mohammadi and F. M. Ghannouchi, "Single RF front-end MIMO transceivers," IEEE Commun. Mag., vol. 49, no. 12, pp. 104-109, Dec. 2011.

[134] P. Banelli et al., "Modulation formats and waveforms for 5G networks: Who will be the heir of OFDM?: An overview of alternative modulation schemes for improved spectral efficiency," IEEE Signal Process. Mag., vol. 31, no. 6, pp. 80-93, Nov. 2014.

[135] D. J. Love, R. W. Heath, V. K. N. Lau, D. Gesbert, B. D. Rao, and M. Andrews, "An overview of limited feedback in wireless communication systems," IEEE J. Sel. Areas Commun., vol. 26, no. 8, pp. 1341-1365, Oct. 2008.

[136] C. B. Chae, A. Forenza, R. W. Heath Jr., M. R. Mckay, and I. B. Collings, "Adaptive MIMO transmission techniques for broadband wireless communication systems," IEEE Commun. Mag., vol. 48, no. 5, pp. 112-118, May 2010.

[137] P. Yang, Y. Xiao, Y. Yi, and S. Li, "Adaptive spatial modulation for wireless MIMO transmission systems," IEEE Commun. Lett., vol. 15, no. 6, pp. 602-604, Jun. 2011.

[138] P. Yang, Y. Xiao, L. Li, Q. Tang, Y. Yu, and S. Li, "Link adaptation for spatial modulation with limited feedback," IEEE Trans. Veh. Technol., vol. 61 , no. 8 , pp. $3808-3813$, Oct. 2012

[139] M. Maleki, H. R. Bahrami, S. Beygi, M. Kafashan, and N. H. Tran, "Space modulation with CSI: Constellation design and performance evaluation," IEEE Trans. Veh. Technol., vol. 62, no. 4, pp. 1623-1634, May 2013.

[140] M. Maleki, H. R. Bahrami, A. Alizadeh, and N. H. Tran, "On the performance of spatial modulation: Optimal constellation breakdown," IEEE Trans. Commun., vol. 62, no. 1, pp. 144-157, Jan. 2014.

[141] C. Masouros and L. Hanzo, "Constellation-randomization achieves transmit diversity for single-RF spatial modulation," IEEE Trans. Veh. Technol., 2015, to be published, doi: 10.1109/TVT.2015.2513380.

[142] A. Garcia, C. Masouros, and L. Hanzo, "Pre-scaling optimization for space shift keying based on semidefinite relaxation," IEEE Trans. Commun., vol. 63, no. 11, pp. 4231-4243, Nov. 2015.

[143] P. Yang, Y. Xiao, B. Zhang, S. Li, M. El-Hajjar, and L. Hanzo, "Power allocation aided spatial modulation for limited-feedback MIMO systems," IEEE Trans. Veh. Technol., vol. 64, no. 5, pp. 2198-2204, May 2015.

[144] P. Yang, Y. Xiao, S. Li, and L. Hanzo, "A low-complexity power allocation algorithm for multiple-input-multiple-output spatial modulation systems," IEEE Trans. Veh. Technol., vol. 65, no. 3, pp. 1819-1825, Mar. 2016, doi: 10.1109/TVT.2015.2410252.

[145] D. Yang, C. Xu, L.-L. Yang, and L. Hanzo, "Transmit-diversity-assisted space-shift keying for colocated and distributed/cooperative MIMO elements," IEEE Trans. Veh. Technol., vol. 60, no. 6, pp. 2864-2869, Jul. 2011.

[146] C. Masouros, "Improving the diversity of spatial modulation in MISO channels by phase alignment," IEEE Commun. Lett., vol. 18, no. 5, pp. 729-732, May 2014.

[147] P. Yang, Y. Xiao, S. Li, and L. Hanzo, "Phase rotation-based precoding for spatial modulation systems," IET Commun., vol. 9, no. 10, pp. 13151323, Jun. 2015.

[148] M. S. Veedu, C. R. Murthy, and L. Hanzo, "Single-RF spatial modulation relying on finite-rate phase-only feedback: Design and analysis," IEEE Trans. Veh. Technol., 2015, to be published, doi: 10.1109/TVT.2015.2424960.

[149] P. Yang, Y. L. Guan, Y. Xiao, M. Di Renzo, and S. Li, "Transmit pre-coding aided spatial modulation maximum the minimum Euclidean distance versus minimum the BER," IEEE Trans. Wireless Commun., vol. 15, no. 3, pp. 2054-2068, Mar. 2016, to be published, doi: 10.1109/TWC.2015.2497692.

[150] H. Li, L. Song, D. Zhu, and M. Lei, "Energy efficiency of large scale MIMO systems with transmit antenna selection," in Proc. IEEE Int. Conf. Commun. (ICC), Jun. 2013, pp. 4641-4645.

[151] R. Kudo, S. M. D. Armour, J. P. McGeehan, and M. Mizoguchi, "A channel state information feedback method for massive MIMO OFDM," IEEE J. Commun. Netw., vol. 15, no. 4, pp. 352-361, Aug. 2013.

[152] E. Başar, Ü. Aygölü, E. Panayırcı, and H. V. Poor, "Performance of spatial modulation in the presence of channel estimation errors," IEEE Commun. Lett., vol. 16, no. 2, pp. 176-179, Feb. 2012.
[153] S. S. Ikki and R. Mesleh, "A general framework for performance 2167 analysis of space shift keying (SSK) modulation in the presence of 2168 Gaussian imperfect estimations," IEEE Commun. Lett., vol. 16, no. 2, 2169 pp. 228-230, Feb. 2012.

[154] S. Sugiura and L. Hanzo, "Effects of channel estimation on spatial mod- 2171 ulation," IEEE Signal Process. Lett., vol. 19, no. 12, pp. 805-808, Dec. 2172 2012.

[155] X. Wu, H. Claussen, M. Di Renzo, and H. Haas, "Channel estimation for 2174 spatial modulation," IEEE Trans. Commun., vol. 62 , no. 12 , pp. $4362-2175$ 4372, Dec. 2014.

[156] Z. Liu, U. Parampalli, and Y. L. Guan, "Optimal odd-length binary Z- 2177 complementary pairs," IEEE Trans. Inf. Theory, vol. 60, no. 9, pp. 5768- 2178 5781, Jul. 2014.

[157] Z. Liu, Y. Li, and Y. L. Guan, "New constructions of general QAM 2180 Golay complementary sequences," IEEE Trans. Inf. Theory, vol. 59, 2181 no. 11, pp. 7684-7692, Nov. 2013.

[158] Z. Liu, Y. L. Guan, and U. Parampalli, "New complete complementary 2183 codes for the peak-to-mean power control in MC-CDMA," IEEE Trans. 2184 Commun., vol. 62, no. 3, pp. 1105-1113, Mar. 2014.

2185

[159] Z. Liu, Y. L. Guan, and H. H. Chen, "Fractional-delay-resilient receiver 2186 for interference-free MC-CDMA communications based on complete 2187 complementary codes," IEEE Trans. Wireless Commun., vol. 14, no. 3, 2188 pp. 1226-1236, Oct. 2014.

2189

[160] M. El-Fandi, I. A. Henderson, J. McGhee, and P. McGlone, 2190 "Uncorrelated multisymbol signals for MIMO system identification," 2191 IEEE Trans. Instrum. Meas., vol. 47, no. 10, pp. 1133-1138, Oct. 1998. 2192

[161] S. Chen, S. Sugiura, and L. Hanzo, "Semi-blind joint channel estimation 2193 and data detection for space-time shift keying systems," IEEE Signal 2194 Process. Lett., vol. 17, no. 12, pp. 993-996, Dec. 2010.

[162] R. Rajashekar, K. V. S. Hari, and L. Hanzo, "Reduced complexity 2196 ML detection and capacity-optimized training for spatial modulation 2197 systems," IEEE Trans. Commun., vol. 62, no. 1, pp. 112-125, Jan. 2014. 2198

[163] M. Wen, Z. Ding, X. Cheng, Y. Bian, H. V. Poor, and B. Jiao, 2199 "Performance analysis of differential spatial modulation with two trans- 2200 mit antennas," IEEE Commun. Lett., vol. 18, no. 3, pp. 475-478, Mar. 2201 2014.

[164] N. Ishikawa and S. Sugiura, "Unified differential spatial modulation," 2203 IEEE Wireless Commun. Lett., vol. 3, no. 4, pp. 337-340, Feb. 2014.2204

[165] Y. Bian, X. Cheng, M. Wen, L. Yang, H. V. Poor, and B. Jiao, 2205 "Differential spatial modulation," IEEE Trans. Veh. Technol., vol. 64, 2206 no. 7, pp. 3262-3268, Jul. 2015.

2207

[166] M. Wen, X. Cheng, Y. Bian, and H. V. Poor, "A low-complexity near- 2208 ML differential spatial modulation detector," IEEE Signal Process. Lett., 2209 vol. 22 , no. 11 , pp. 1834-1838, Nov. 2015.

[167] P. A. Martin, "Differential spatial modulation for APSK in time-varying 2211 fading channels," IEEE Commun. Lett., vol. 19, no. 7, pp. 1261-1264, 2212 Jul. 2015.

[168] L. Xiao, P. Yang, X. Lei, Y. Xiao, and S. Li, "A low-complexity detec- 2214 tion scheme for differential spatial modulation," IEEE Commun. Lett., 2215 vol. 19, no. 9, pp. 1516-1519, Sep. 2015.

2216

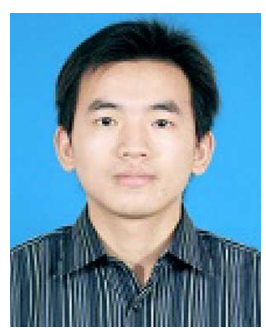

Ping Yang received the Ph.D. degree from the 2217 University of Electronic Science and Technology of 2218 China (UESTC), Chengdu, China, in 2013. From 2219 September 2012 to September 2013, he was a Visiting 2220 Student at the School of Electronics and Computer 2221 Science, University of Southampton, Southampton, 2222 UK. From May 2014, he is a Research Fellow in 2223 EEE of NTU, Singapore. Also, he is an Assistant 2224 Professor with UESTC. His research interests include 2225 MIMO/OFDM, machine learning, life science, and 2226 communication signal processing.

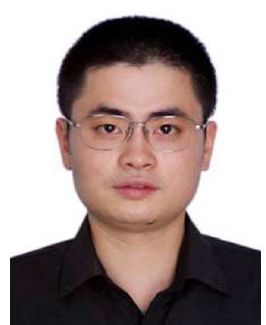

Yue Xiao received the Ph.D. degree in communi- 2228 cation and information systems from the University 2229 of Electronic Science and Technology of China, 2230 Chengdu, China, in 2007. He is now an Full 2231 Professor with the University of Electronic Science 2232 and Technology of China. He has authored more than 2233 30 international journals and been involved in several 2234 projects in Chinese Beyond 3G Communication R\&D 2235 Program. His research interests include wireless and 2236 mobile communications. 


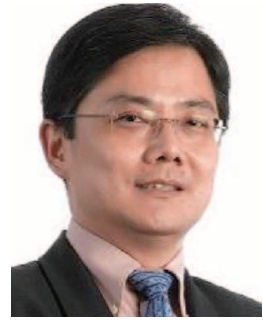

Yong Liang Guan (M'99) received the B.Eng degree (with first class Hons.) from the National University of Singapore, Singapore, in 1991, and the Ph.D. degree from the Imperial College of Science, Technology and Medicine, University of London, London, U.K., in 1997. He is now an Associate Professor with the School of Electrical and Electronic Engineering, Nanyang Technological University, Singapore. His research interests include modulation, coding and signal processing for communication, information security and storage systems.

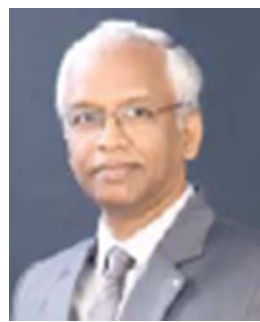

K. V. S. Hari (M'92-SM'97-F'15) received the B.E. degree from Osmania University, Hyderabad, India, in 1983; the M.Tech. degree from the Indian Institute of Technology Delhi, New Delhi, USA, in 1985; and the Ph.D. degree from the University of California at San Diego, La Jolla, CA, USA, in 1990. Since 1992, he has been with the Department of Electrical Communication Engineering, Indian Institute of Science, Bangalore, Bangalore, India, where he is currently a Professor and coordinates the activities of the Statistical Signal Processing Laboratory. He is also, currently, an Affiliated Professor with the School of Electrical Engineering, KTH-Royal Institute of Technology.

His research interests include the development of signal processing algorithms for MIMO wireless communication systems, sparse signal recovery problems, indoor positioning, assistive technologies for the elderly, and visual neuroscience.

He has been a Visiting Faculty Member at Stanford University, Stanford, CA, USA; KTH-Royal Institute of Technology, Stockholm, Sweden; and Aalto University, Espoo, Finland (formerly Helsinki University of Technology), During his work at Stanford University, he worked on MIMO wireless channel modeling and is the coauthor of the WiMAX standard on wireless channel models for fixed-broadband wireless communication systems, which proposed the Stanford University Interim (SUI) channel models. He also worked with DLRL, Hyderabad, India, and the Research and Training Unit for Navigational Electronics, Osmania University, Hyderabad, India. He is currently an Editor of EURASIP Journal Signal Processing (Elsevier) and the Senior Associate Editor of Indian Academy of Sciences Journal Sadhana (Springer).

He was the recipient of the IETE S.V.C. Aiya Award for excellence in Telecom Education and the Distinguished Almnus Award from the Osmania University College of Engineering, Hyderabad, India. He is an Academic Entrepreneur and a Co-Founder of the company ESQUBE Communication Solutions, Bangalore, India.

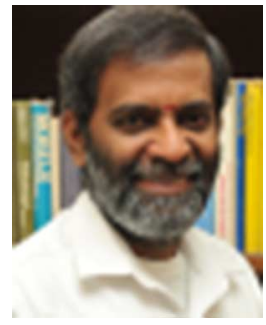

A. Chockalingam (S'92-M'93-SM'98) was born in Rajapalayam, Tamil Nadu, India. He received the B.E. degree (Hons.) in electronics and communication engineering from the P.S.G. College of Technology, Coimbatore, India, in 1984, the M.Tech. degree in electronics and electrical communications engineering (with specialization in satellite communications) from the Indian Institute of Technology, Kharagpur, Kharagpur, India, in 1985, and the Ph.D. degree in electrical communication engineering (ECE) from the Indian Institute of Science (IISc), Bangalore, India, in 1993. During 1986 to 1993, he worked with the Transmission R\&D Division, Indian Telephone Industries Limited, Bangalore, India. From December 1993 to May 1996, he was a Postdoctoral Fellow and an Assistant Project Scientist with the Department of Electrical and Computer Engineering, University of California, San Diego, La Jolla, CA, USA. From May 1996 to December 1998, he served Qualcomm, Inc., San Diego, CA, USA, as a Staff Engineer/Manager with the Systems Engineering Group. In December 1998, he joined the Faculty of the Department of ECE, IISc, Bangalore, India, where he is a Professor, working in the area of wireless communications and networking.

Dr. Chockalingam served as an Associate Editor of the IEEE Transactions on Vehicular Technology, and as an Editor of the IEEE TRANSACtions on Wireless Communications. He served as the Guest Editor for the IEEE Journal ON SELECTED AREAS IN Communications (Special Issue on Multiuser Detection for Advanced Communication Systems and Networks), and for the IEEE JOURNAL OF Selected Topics in Signal Processing (Special Issue on Soft Detection on Wireless Transmission). He is a Fellow of the Indian National Academy of Engineering, the National Academy of Sciences, India, the Indian National 2312 Science Academy, and the Indian Academy of Sciences. He was the recipient 2313 of the J. C. Bose National Fellowship and the Swarnajayanti Fellowship from 2314 the Department of Science and Technology, Government of India.

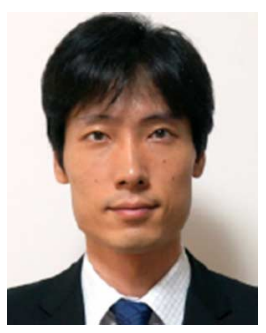

Shinya Sugiura (M'06-SM'12) received the B.S. 2316 and M.S. degrees in aeronautics and astronautics 2317 from Kyoto University, Kyoto, Japan, in 2002 and 2318 2004, respectively, and the Ph.D. degree in electron- 2319 ics and electrical engineering from the University of 2320 Southampton, Southampton, U.K., in 2010.

From 2004 to 2012, he was a Research Scientist 2322 with Toyota Central Research and Development 2323 Laboratories, Inc., Aichi, Japan. Since 2013, he has 2324 been an Associate Professor with the Department 2325 of Computer and Information Sciences, Tokyo 2326 University of Agriculture and Technology, Tokyo, Japan, where he is the Head 2327 of the Wireless Communications Research Group. He authored or coauthored 2328 over 70 refereed research publications, including 40 IEEE journal and magazine 2329 papers. His research interests include wireless communications, networking, 2330 signal processing, and antenna technology.

Dr. Sugiura was also certified as an Exemplary Reviewer of IEEE 2332 COMMUNiCATIONS LETTERS in 2013 and 2014. He was the recipient of a 2333 number of awards, including the 14th Funai Information Technology Award 2334 (First Prize) from the Funai Foundation in 2015, the 28th Telecom System 2335 Technology Award from the Telecommunications Advancement Foundation in 2336 2013, the Sixth IEEE Communications Society Asia-Pacific Outstanding Young 2337 Researcher Award in 2011, the 13th Ericsson Young Scientist Award in 2011, 2338 and the 2008 IEEE Antennas and Propagation Society Japan Chapter Young 2339 Engineer Award.

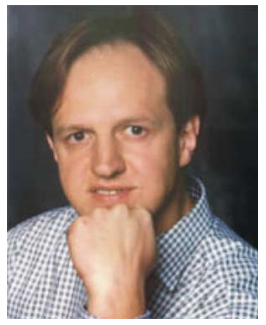

Harald Haas (S'98-AM'00-M'03) received the 2341 Ph.D. degree from the University of Edinburgh, 2342 Edinburgh, U.K., in 2001. He currently holds the 2343 Chair of Mobile Communications at the University 2344 of Edinburgh. His research interests include optical 2345 wireless communications, hybrid optical wireless and 2346 RF communications, spatial modulation, and interfer- 2347 ence coordination in wireless networks. He was an 2348 Invited Speaker at TED Global 2011, and his talk has 2349 been watched online more than 1.5 million times. He 2350 is Co-Founder and Chief Scientific Officer (CSO) of 2351 pureLiFi Ltd. He holds 31 patents and has more than 30 pending patent appli- 2352 cations. He has authored 300 conference and journal papers including a paper 2353 in Science. He was the corecipient of the Best Paper Award at the IEEE 2354 Vehicular Technology Conference in Las Vegas, NV, USA, in 2013. In 2012, 2355 he was the only recipient of the Prestigious Established Career Fellowship 2356 from the Engineering and Physical Sciences Research Council (EPSRC) within 2357 Information and Communications Technology in the U.K. He was also the 2358 recipient of the Tam Dalyell Prize 2013 awarded by the University of Edinburgh 2359 for excellence in engaging the public with science. In 2014, he was selected 2360 by EPSRC as one of ten Recognising Inspirational Scientists and Engineers 2361 (RISE) Leaders.

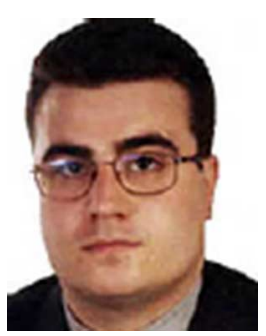

Marco Di Renzo (S'05-AM'07-M'09-SM'14) was 2363 born in L'Aquila, Italy, in 1978. He received the 2364 Laurea (cum laude) and Ph.D. degrees in electrical 2365 and information engineering from the Department of 2366 Electrical and Information Engineering, University of 2367 L'Aquila, L'Aquila, Italy, in April 2003 and January 2368 2007, respectively, the Habilitation à Diriger des 2369 Recherches (HDR) degree from the University of 2370 Paris-Sud XI, Paris, France, in October 2013. Since 2371 January 2010, he has been a Tenured Academic 2372 Researcher ("Chargé de Recherche Titulaire") with 2373 the French National Center for Scientific Research (CNRS), as well as a fac- 2374 ulty member of the Laboratory of Signals and Systems (L2S), a Joint Research 2375 Laboratory of the CNRS, the École Supérieure d'Électricité (SUPÉLEC) and 2376 the University of Paris-Sud XI, Paris, France. His research interests include 2377 wireless communications theory.

Dr. Di Renzo was the recipient of a special mention for the outstand- 2379 ing five-year (1997-2003) academic career, University of L'Aquila, Italy; the 2380 THALES Communications Fellowship (2003-2006), University of L'Aquila, 2381 Italy; the 2004 Best Spin-Off Company Award, Abruzzo Region, Italy; 2382 the 2006 DEWS Travel Grant Award, University of L'Aquila; the 20082383 Torres Quevedo Award, Ministry of Science and Innovation, Spain; the 2384 
"Dérogation pour l'Encadrement de Thèse" (2010), University of Paris-Sud XI, France; the 2012 IEEE CAMAD Best Paper Award; the 2012 IEEE Wireless COMMUNiCATIONS LeTters Exemplary Reviewer Award; the 2013 IEEE VTC-Fall Best Student Paper Award; the 2013 Network of Excellence NEWCOM\# Best Paper Award; the 2013 IEEE TRANSACTIONS on Vehicular Technology Top Reviewer Award; the 2013 IEEECOMSOC Best Young Researcher Award for Europe, Middle East and Africa (EMEA Region); and the 2014 IEEE ICNC Single Best Paper Award Nomination (Wireless Communications Symposium). Currently, he serves an an Editor of the IEEE COMMUNICATIONS LETTERS and the IEEE TRANSACTIONS ON COMMUNiCATIONS (Wireless CommunicationsHeterogeneous Networks Modeling and Analysis).

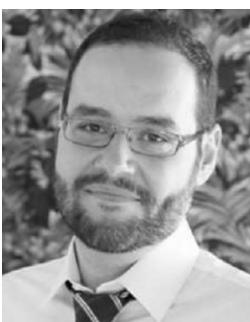

Christos Masouros (M'06-SM'14) received the diploma degree in electrical and computer engineering from the University of Patras, Patron, Greece, in 2004, the M.Sc. degree by research, and the $\mathrm{Ph} . \mathrm{D}$. degree in electrical and electronic engineering from the University of Manchester, Manchester, U.K., in 2006 and 2009, respectively. He is currently a Lecturer with the Department of Electrical and Electronic Engineering, University College London, London, U.K. He has previously held a Research Associate position with the University of Manchester and a Research Fellow position in Queen's University Belfast, Belfast, U.K.

He holds a Royal Academy of Engineering Research Fellowship 2011-2016 and is the Principal Investigator of the EPSRC project EP/M014150/1 on large scale antenna systems. His research interests include wireless communications and signal processing with particular focus on green communications, large scale antenna systems, cognitive radio, interference mitigation techniques for MIMO and multicarrier communications. He is an Associate Editor for the IEEE Communications LetTers. He was the recipient of the Best Paper Award in the IEEE GLOBECOM 2015.

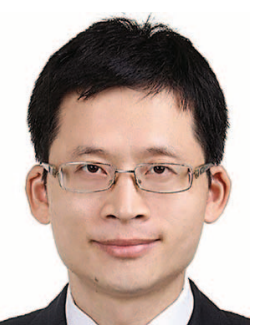

Zilong Liu received the bachelor's degree in electronics and information engineering from Huazhong University of Science and Technology (HUST), Wuhan, China, the master's degree in electronic engineering from Tsinghua University, Beijing, China, and the $\mathrm{Ph} . \mathrm{D}$. degree in electrical and electronic engineering, Nanyang Technological University (NTU), Singapore, in 2004, 2007, and 2014, respectively. Since July 2008, he has been with the School of Electrical and Electronic Engineering, NTU, first as a Research Associate and since November 2014, as a Research Fellow. He was a Visitor at the University of Melbourne (UoM), Parkville, Vic., Australia, from May 2012 to February 2013 (hosted by prof. Udaya Parampalli), and a Visiting Ph.D. Student at the Hong Kong University of Science and Technology (HKUST) from June 2013 to July 2013 (hosted by prof. Wai Ho Mow). He research interests include coding and signal processing for various communication systems, with emphasis on signal design and algebraic coding, error correction codes, optimal channel estimation, robust/efficient multiuser communications, and physical layer receiver design.

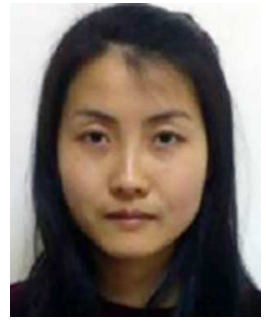

Lixia Xiao received the B.E. and M.E. degrees from 2436 the University of Electronic Science and Technology 2437 of China (UESTC), Chengdu, China, in 2010 and 2438 2013, respectively. She is currently pursing the Ph.D. 2439 degree at the National Key Laboratory of Science 2440 and Technology on Communications, UESTC. Her 2441 research interests include wireless communications, 2442 communication theory, signal detection, and perfor- 2443 mance analysis of wireless communication systems. 2444

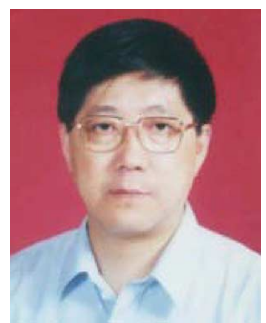

Shaoqian Li (F'16) received the B.S.E. degree in 2445 communication technology from Northwest Institute 2446 of Telecommunication (Xidian University), Xi'an, 2447 China, in 1982, and the M.S.E. degree in communica- 2448 tion system from the University of Electronic Science 2449 and Technology of China (UESTC), Chengdu, China, 2450 in 1984. He is a Professor, a Ph.D. Supervisor, 2451 the Director of the National Key Laboratory of 2452 Communication, UESTC, and a Member of the 2453 National High Technology R\&D Program (863 2454 Program) Communications Group. His research inter- 2455 ests include wireless communication theory, anti-interference technology for 2456 wireless communications, spread-spectrum and frequency-hopping technology, 2457 mobile and personal communications.

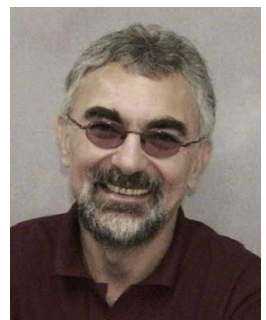

Lajos Hanzo (M'91-SM'92-F'04) received the 2459 degree in electronics in 1976 and the doctorate degree 2460 in 1983. In 2009, he was received the Honorary 2461 Doctorate "Doctor Honoris Causa" by the Technical 2462 University of Budapest. During his 37-year career 2463 in telecommunications, he has held various research 2464 and academic posts in Hungary, Germany, and the 2465 U.K. Since 1986, he has been with the School of 2466 Electronics and Computer Science, University of 2467 Southampton, Southampton, U.K., where he holds 2468 the chair in telecommunications. He has successfully 2469 supervised 80+ Ph.D. students, coauthored 20 John Wiley/IEEE Press books 2470 on mobile radio communications totalling in excess of 10,000 pages, authored 2471 1400+ research entries at IEEE Xplore, acted both as TPC and the General 2472 Chair of IEEE conferences, presented keynote lectures and has been awarded 2473 a number of distinctions. Currently, he is directing a 100-strong academic 2474 research team, working on a range of research projects in the field of wire- 2475 less multimedia communications sponsored by industry, the Engineering and 2476 Physical Sciences Research Council (EPSRC) U.K., the European Research 2477 Council's Advanced Fellow Grant and the Royal Society's Wolfson Research 2478 Merit Award. He is an enthusiastic supporter of industrial and academic liaison 2479 and he offers a range of industrial courses. He is also a Governor of the IEEE 2480 VTS. From 2008 to 2012, he was the Editor-in-Chief of the IEEE Press and a 2481 Chaired Professor also at Tsinghua University, Beijing, China. His research is 2482 supported by the European Research Council's Senior Research Fellow Grant. 2483 He is a Fellow of the REng, IET, EURASIP, and DSc. He has over 19,000 2484 citations. 